\title{
15. X-RAY MINERALOGY STUDIES OF LEG 10 CORES IN THE GULF OF MEXICO ${ }^{1}$
}

\author{
Ivar Zemmels and Harry E. Cook, University of California, Riverside, California
}

Semiquantitative determinations of the mineral composition of bulk samples and the $<2 \mu$ fraction and qualitative determinations in the $2-20 \mu$ fraction were performed according to the methods described in the reports of Legs 1 and 2 and in Appendix III of Volume IV (Fig. 4). The mineral analysis of the $2-20 \mu$ and $<2 \mu$ fractions were performed on $\mathrm{CaCO}_{3}$-free residues.

The results are presented in Tables 1 to 13 and also in Figures 1 to 26 . The sediment ages, the lithologic units, and the nomenclature of the sediment types used in Figures 1 to 26 are from the DSDP Leg 10 hole summaries. The samples submitted for X-ray diffraction analysis from Leg 10 are listed in Table 14. The sample depths below the sea floor in the last column of Table 14 identify the samples as they are reported in Tables 1 to 13 and Figures 1 to 26. Single depths indicate single sediment samples. Brackets are used to indicate the samples which are combined into one composite sample; the depths give the range of the combined interval. The procedure on Leg $10 \mathrm{sam}$ ples was to combine all of the sediment samples from one core into a single sample. The usual procedure for Legs 3 to 9 and Legs 11 et seq. has been to combine only samples of similar lithology which occur in one core.

No semiquantitative determinations of the mineral concentrations were made for the silt fractions $(2-20 \mu)$ as in Legs 8, 9, and 11, but selected minerals that commonly have an authigenic origin were sought and are reported in Tables 1 to 13 on a ranked, semiqualitative scale. The

'Institute of Geophysics and Planetary Physics, University of California, Riverside, California. Contribution No. IGPP-UCR-72-31. minerals sought were: barite, phillipsite, clinoptilolite, erionite, dolomite, siderite, rhodochrosite, geothite, hematite, magnetite, cristobalite, pyrite, and apatite. These minerals were ranked on an approximate semiqualitative scale as follows: major (M), $<25$ per cent; abundant (A), 25-8 per cent; present (P), 8-2 per cent; and trace (T), > 2 per cent. The values of the diffuse scattering, amorphous scattering, and the two most abundant minerals are also included to help characterize the sample.

The use of drilling mud which contains montmorillonite and barite was as follows: Hole 85, after Core 4 and after Core 5; Hole 85A, before Core 1; Hole 86, after Core 11; Hole 87, before and after Core 1; Hole 89, after Core 3 and after Core 5; Hole 91, after Core 10. Generally the affected cores are free of any detectable contamination. However, the composited samples from Hole 87, Core 1 and Hole 89 , Core 4 probably contain some montmorillonite from the drilling mud.

Between the X-ray diffraction analysis of the bulk samples and the $<2 \mu$ fractions of Leg 10 , several changes were made to the diffractometer. The lithium fluoride receiving monochrometer crystal was replaced by a graphite crystal and the slit system was modified. This resulted in a higher diffraction intensity and better resolution of the diffraction peaks. Calibration of the new instrument configuration by means of mineral standards was performed which resulted in the modification of the parameters in the X-ray diffraction data reduction program (MINLOG). The data of both sets of samples should be compatible within the limits of accuracy of our method. The parameters used for the semiquantitative determination of mineral concentration in bulk samples and $<2 \mu$ fractions are given in Table 15. The ranked semiqualitative data for the $2-20 \mu$ fractions were derived by using the new parameters. 
TABLE 1

Results of X-Ray Diffraction Analyses from Site $85^{\mathrm{a}}$

\begin{tabular}{|c|c|c|c|c|c|c|c|c|c|c|c|c|c|c|c|c|c|c|}
\hline Core & $\begin{array}{c}\text { Cored } \\
\text { Interval } \\
\text { Below Sea } \\
\text { Floor (m) }\end{array}$ & $\begin{array}{l}\text { Sample Depth } \\
\text { Below Sea } \\
\text { Floot } \\
\text { (m) }\end{array}$ & Diff. & Amor. & Calc. & Dolo. & Arag. & Quar. & $\mathrm{K}-\mathrm{Fe}$ & Paly. & Kaol. & Mica & Chlo. & Mont. & Hali. & Clin. & Pyri. & Paly. \\
\hline \multicolumn{19}{|c|}{ Bulk Samples } \\
\hline 1 & $19-28$ & $19.05-27.51$ & 78.0 & 42.0 & 10.6 & 8.0 & - & 23.8 & - & 7.3 & 2.7 & 35.8 & 4.2 & 5.7 & 1.5 & - & & \\
\hline 2 & $48-57$ & $48.08-51.77$ & 80.5 & 44.5 & 11.9 & 8.8 & - & 13.0 & - & 6.2 & 1.6 & 44.5 & 4.5 & 5.3 & 4.2 & - & & \\
\hline 3 & $99-108$ & $99.12-107.72$ & 78.6 & 42.6 & 9.3 & 7.4 & - & 15.4 & - & 6.2 & 3.1 & 46.3 & 3.7 & 6.1 & 2.5 & - & & \\
\hline 4 & $189-198$ & $189.51-190.43$ & 78.1 & 42.1 & 22.9 & 4.1 & 25.0 & 10.8 & - & 6.5 & - & 21.6 & 1.7 & 7.0 & - & - & & \\
\hline 5 & $210-212$ & $210.69-212.60$ & 44.3 & 8.3 & 11.9 & 4.3 & - & 56.3 & 11.2 & 12.8 & - & 2.9 & - & - & - & - & & \\
\hline $2 \mathrm{~A}$ & $282-302$ & 292.00 & 68.0 & 32.0 & - & 35.3 & - & 5.3 & 3.2 & 5.6 & - & 26.5 & 1.4 & 8.6 & - & 13.6 & & \\
\hline \multicolumn{19}{|c|}{ 2-20 $\mu$ Fraction } \\
\hline 1 & $19-28$ & $19.05-27.51$ & 63.4 & 42.8 & & & & $\dagger$ & & & & * & & & & & $\mathbf{T}$ & \\
\hline 2 & $48-57$ & $48.08-51.77$ & 67.2 & 48.8 & & $\mathrm{P}$ & & * & & & & $\dagger$ & & & & & & \\
\hline 3 & $99-108$ & $99.12-107.72$ & 65.4 & 46.0 & & $P$ & & $\dagger$ & & & & * & & & & & & \\
\hline 4 & $189-198$ & $189.51-190.43$ & 70.9 & 54.5 & & & & $\dagger$ & & & & * & & & & & & $\mathrm{T}$ \\
\hline 5 & $210-212$ & $210.69-212.60$ & 59.9 & 37.3 & & & & * & & & & $\dagger$ & & & & & & \\
\hline $2 \mathrm{~A}$ & $282-302$ & 292.0 & 64.7 & 44.9 & & $P$ & & $\dagger$ & & & & $*$ & & & & & $\mathbf{T}$ & $\mathbf{P}$ \\
\hline \multicolumn{19}{|c|}{$<2 \mu$ Fraction } \\
\hline 1 & $19-28$ & $19.05-27.51$ & 71.8 & 55.9 & & & & 13.1 & - & 1.7 & 2.7 & 52.1 & 4.0 & 26.0 & & - & & \\
\hline 2 & $48-57$ & $48.08-51.79$ & 67.4 & 49.0 & & & & 19.9 & 2.8 & 4.5 & 1.5 & 60.4 & 4.4 & 6.1 & & - & & \\
\hline 3 & $99-108$ & $99.12-107.72$ & 72.2 & 56.5 & & & & 9.7 & - & 2.5 & 3.6 & 56.5 & 4.6 & 23.1 & & - & & \\
\hline 4 & $189-198$ & $189.51-190.43$ & 77.8 & 65.2 & & & & 13.4 & 14.8 & 2.5 & 2.5 & 35.9 & 3.8 & 27.0 & & - & & \\
\hline 5 & $210-212$ & $210.69-212.60$ & 79.5 & 67.9 & & & & 16.3 & 4.8 & 12.2 & 2.9 & 46.2 & 5.1 & 12.2 & & - & & \\
\hline $2 \mathrm{~A}$ & $282-302$ & 292.00 & 75.1 & 61.1 & & & & 5.6 & - & 3.0 & - & 35.6 & 3.3 & 15.1 & & 37.4 & & \\
\hline
\end{tabular}

${ }^{a}$ No entry in column indicates not determined

* 1st Major Constituents

$\dagger_{2 \text { nd Major Constituents }}$

P - Present $(8-2 \%)$

T - Trace $(<2 \%)$

TABLE 2

Results of X-Ray Diffraction Analy ses from Site $86^{\mathrm{a}}$

\begin{tabular}{|c|c|c|c|c|c|c|c|c|c|c|c|c|c|c|c|c|c|c|}
\hline Core & $\begin{array}{c}\text { Cored } \\
\text { Interval } \\
\text { Below Sea } \\
\text { Floor }(\mathrm{m})\end{array}$ & $\begin{array}{l}\text { Sample Depth } \\
\text { Below Sea } \\
\text { Floor } \\
\text { (m) }\end{array}$ & Diff. & Amor. & Calc. & Dolo. & Arag. & Quar. & $\mathrm{K}-\mathrm{Fe}$ & Plag. & Kaol. & Mica & Chlo. & Mont. & Paly. & Clin. & Pyri. Augi. & Gyps. \\
\hline \multicolumn{19}{|c|}{ Bulk Samples } \\
\hline 1 & $13-17$ & $\begin{array}{c}13.09 \\
13.18-16.76\end{array}$ & $\begin{array}{l}68.1 \\
68.7\end{array}$ & $\begin{array}{l}32.1 \\
32.7\end{array}$ & $\begin{array}{l}55.9 \\
38.9\end{array}$ & $\overline{1.3}$ & $\begin{array}{l}17.1 \\
30.6\end{array}$ & $\begin{array}{l}6.7 \\
8.2\end{array}$ & - & $\begin{array}{l}1.8 \\
1.7\end{array}$ & $\begin{array}{l}3.3 \\
1.5\end{array}$ & $\begin{array}{l}10.9 \\
10.4\end{array}$ & $\begin{array}{l}3.3 \\
1.1\end{array}$ & $\overline{5.6}$ & - & - & & \\
\hline 2 & $52-61$ & 52.60 & 69.8 & 33.8 & 53.8 & - & 11.7 & 7.4 & - & 2.3 & 1.0 & 16.9 & 1.4 & 5.5 & - & - & & \\
\hline 3 & $161-170$ & $161.20-169.26$ & 65.9 & 29.9 & 69.6 & - & 9.1 & 3.9 & - & - & 1.1 & 10.9 & - & 4.6 & - & - & & \\
\hline 4 & $257-266$ & $257.30-263.75$ & 60.9 & 24.9 & 81.1 & - & - & 3.0 & - & - & 1.5 & 7.0 & - & 4.5 & 2.7 & - & & \\
\hline 5 & $371-380$ & $372.16-376.26$ & 54.4 & 18.4 & 99.3 & - & - & 0.7 & - & - & - & - & - & - & - & - & & \\
\hline 7 & $498-507$ & $499.69-504.77$ & 53.8 & 17.8 & 95.0 & - & - & 1.3 & - & - & - & - & - & - & - & 3.7 & & \\
\hline 8 & $508-517$ & $509.37-513-19$ & 74.5 & 38.5 & 76.4 & - & - & 2.7 & 2.1 & 3.1 & - & 7.2 & - & 9.0 & - & - & & \\
\hline 9 & $551-560$ & $551.05-552.60$ & 55.8 & 19.8 & 86.8 & - & - & 2.2 & 1.8 & - & - & 5.4 & - & 3.5 & - & - & & \\
\hline
\end{tabular}


TABLE 2 - Continued

\begin{tabular}{|c|c|c|c|c|c|c|c|c|c|c|c|c|c|c|c|c|c|c|}
\hline Core & $\begin{array}{l}\text { Cored } \\
\text { Interval } \\
\text { Below Sea } \\
\text { Floor (m) }\end{array}$ & $\begin{array}{l}\text { Sample Depth } \\
\text { Below Sea } \\
\text { Floor } \\
\text { (m) }\end{array}$ & Diff. & Amor. Calc. & Dolo. & Arag. & Quar. & $\mathrm{K}-\mathrm{Fe}$ & Plag. & Kaol. & Mica & Chlo. & Mont. & Paly. & Clin. & Pyri. & Augi. & Gyps. \\
\hline \multicolumn{19}{|c|}{ 2-20 $\mu$ Fraction } \\
\hline 1 & $13-17$ & $\begin{array}{c}13.09 \\
13.8-16.76\end{array}$ & $\begin{array}{l}74.1 \\
73.9\end{array}$ & $\begin{array}{l}59.5 \\
59.3\end{array}$ & $\mathbf{T}$ & & $\begin{array}{l}\dagger \\
\dagger\end{array}$ & & & & * & & & $\begin{array}{l}\mathrm{P} \\
\mathrm{T}\end{array}$ & & & & \\
\hline 2 & $52-61$ & 52.60 & 68.7 & 51.1 & & & $\dagger$ & & & & * & & & & & $\mathrm{T}$ & & \\
\hline 3 & $161-170$ & $161.20-169.26$ & 68.8 & 51.2 & $\mathbf{P}$ & & $\dagger$ & & & & * & & & & & $P$ & & \\
\hline 4 & $257-266$ & $257.30-263.75$ & 79.3 & 67.6 & & & * & & & & $\dagger$ & & & $\mathrm{T}$ & $\mathrm{T}$ & $P$ & & \\
\hline 5 & $371-380$ & $372.16-376.26$ & 90.4 & 85.1 & & & $*$ & & & & $\dagger$ & & & & $\mathrm{T}$ & & & \\
\hline 7 & $440-449$ & $499.69-504.77$ & 70.9 & 54.5 & & & & & & & $\dagger$ & & & $\mathrm{P}$ & $*$ & & & \\
\hline 8 & $498-507$ & $509.39-513.19$ & 94.0 & 90.6 & & & $\dagger$ & & & & * & & & $\mathrm{T}$ & & & & \\
\hline 9 & $508-517$ & $551.05-552.60$ & 70.2 & 53.4 & & & $\dagger$ & & & & * & & & & & & $P$ & \\
\hline \multicolumn{19}{|c|}{$<2 \mu$ Fraction } \\
\hline 1 & $13-17$ & $\begin{array}{c}13.09 \\
13.8-16.76\end{array}$ & $\begin{array}{l}80.0 \\
76.8\end{array}$ & $\begin{array}{l}68.7 \\
63.7\end{array}$ & & & $\begin{array}{r}6.3 \\
15.3\end{array}$ & - & $\begin{array}{l}1.6 \\
4.8\end{array}$ & $\begin{array}{l}8.6 \\
5.5\end{array}$ & $\begin{array}{l}43.4 \\
27.1\end{array}$ & $\overline{3.6}$ & $\begin{array}{l}40.1 \\
42.4\end{array}$ & - & - & & & $\overline{1.2}$ \\
\hline 2 & $52-61$ & 52.60 & 76.0 & 62.5 & & & 10.6 & - & - & 11.8 & 37.7 & - & 39.6 & - & - & & & - \\
\hline 3 & $161-170$ & $161.20-169.26$ & 74.7 & 60.5 & & & 9.0 & - & - & 2.3 & 28.9 & 4.0 & 54.8 & - & - & & & 1.0 \\
\hline 4 & $257-266$ & $257.30-263.75$ & 74.3 & 59.9 & & & 7.9 & - & - & 3.7 & 24.1 & 1.8 & 43.0 & 19.3 & - & & & - \\
\hline 5 & $371-380$ & $372.16-376.26$ & 78.4 & 66.2 & & & 12.7 & - & - & 1.7 & 18.4 & 4.0 & 63.2 & - & - & & & - \\
\hline 7 & $498-507$ & $499.69-504.77$ & 75.8 & 62.3 & & & 11.1 & - & 7.9 & - & 13.2 & - & 31.1 & 13.5 & 19.8 & & & 2.5 \\
\hline 8 & $508-517$ & $509.37-513.19$ & 87.6 & 80.7 & & & 15.4 & 27.0 & - & 4.4 & 21.6 & - & 34.1 & - & - & & & - \\
\hline 9 & $551-560$ & $551.05-552.60$ & 78.3 & 66.0 & & & 10.9 & 14.4 & - & - & 18.7 & 6.1 & 24.0 & 24.2 & - & & & 1.7 \\
\hline
\end{tabular}

${ }^{\mathrm{a}}$ No entry in column indicates not determined

* 1st Major Constituents

$\dagger^{2}$ nd Major Constituents

P - Present (8-2\%)

T - Trace $(<2 \%)$

TABLE 3

Results of X-Ray Diffraction Analyses from Site $87^{\mathrm{a}}$

\begin{tabular}{|c|c|c|c|c|c|c|c|c|c|c|c|c|c|c|c|c|}
\hline Core & $\begin{array}{c}\text { Cored } \\
\text { Interval } \\
\text { Below Sea } \\
\text { Floor (m) }\end{array}$ & $\begin{array}{l}\text { Sample Depth } \\
\text { Below Sea } \\
\text { Floor } \\
\text { (m) }\end{array}$ & Diff. & Amor. & Calc. & Dolo. & Quar. & Plag. & Mica & Chlo. & Mont. & Clin. & $\mathrm{K}-\mathrm{Fe}$ & Amph. & Pyri. & Paly. \\
\hline \multicolumn{17}{|c|}{ Bulk Samples } \\
\hline 1 & $648-657$ & $649.05-650.41$ & 68.9 & 32.9 & 5.1 & 1.8 & 18.2 & 25.6 & 33.2 & 2.1 & 4.9 & 2.4 & 4.9 & 1.5 & & \\
\hline \multicolumn{17}{|c|}{$2-20 \mu$ Fraction } \\
\hline 1 & $0-6$ & $649.05-650.41$ & 75.6 & 61.8 & & & & $\dagger$ & $*$ & & & $P$ & & & $P$ & $\mathrm{~T}$ \\
\hline \multicolumn{17}{|c|}{$<2 \mu$ Fraction } \\
\hline 1 & $648-657$ & $649.05-650.41$ & 79.1 & 67.3 & & & 7.4 & 2.2 & 25.3 & 3.4 & 45.0 & 1.8 & & & 1.7 & 12.2 \\
\hline \multicolumn{17}{|c|}{$\begin{array}{l}\text { a No entry in column indicates not determined } \\
{ }^{*} \text { 1st Major Constituents } \\
+2 \text { nd Major Constituents } \\
\text { P - Present }(8-2 \%) \\
\text { T - Trace }(<2 \%)\end{array}$} \\
\hline
\end{tabular}


TABLE 4

Results of X-Ray Diffraction Analyses from Site $88^{\mathrm{a}}$

\begin{tabular}{|c|c|c|c|c|c|c|c|c|c|c|c|c|c|c|c|c|}
\hline Core & $\begin{array}{c}\text { Cored } \\
\text { Intervai } \\
\text { Below Sea } \\
\text { Floor (m) }\end{array}$ & $\begin{array}{l}\text { Sample Depth } \\
\text { Below Sea } \\
\text { Floor } \\
\text { (m) }\end{array}$ & Diff. & Amor. & Calc. & Dolo. & Quar. & Plag. & Kaol. & Mica & Chlo. & Mont. & Paly. & Hali. & Clin. & Pyri. \\
\hline \multicolumn{17}{|c|}{ Bulk Samples } \\
\hline 1 & $0-6$ & $0.27-5.37$ & 75.7 & 39.7 & 35.0 & - & 14.0 & 5.5 & 3.2 & 20.4 & - & 15.4 & 6.5 & - & & \\
\hline 2 & $51-60$ & $51.19-59.28$ & 74.0 & 38.0 & 26.4 & - & 12.9 & 9.3 & 7.1 & 23.8 & - & 11.3 & 7.4 & 2.0 & & \\
\hline 3 & 98-104 & $99.10-103.90$ & 72.3 & 36.3 & 43.8 & - & 11.5 & 3.8 & 3.3 & 17.2 & - & 11.2 & 8.2 & - & & \\
\hline 4 & $104-108$ & $104.00-108.00$ & 73.7 & 37.7 & 52.6 & - & 11.1 & 3.8 & 1.9 & 20.7 & 1.1 & 8.1 & - & - & & \\
\hline 5 & $128-135$ & $128.05-136.41$ & 69.5 & 33.5 & 61.2 & 1.5 & 7.2 & 7.0 & 1.7 & 11.1 & - & 9.4 & - & - & & \\
\hline \multicolumn{17}{|c|}{ 2-20 $\mu$ Fraction } \\
\hline 1 & $0-6$ & $0.27-5.37$ & 64.3 & 44.3 & & & $*$ & & & $\dagger$ & & & & & $\mathrm{T}$ & $\mathrm{T}$ \\
\hline 2 & $51-61$ & $51.19-59.28$ & 73.1 & 57.9 & & & * & & & $\dagger$ & & & $\mathrm{T}$ & & $\mathrm{T}$ & $P$ \\
\hline 3 & $98-104$ & $99.10-103.90$ & 70.8 & 54.4 & & & * & & & $\dagger$ & & & $\mathrm{T}$ & & & $P$ \\
\hline 4 & $104-108$ & $104.00-108.00$ & 74.6 & 60.3 & & & $\dagger$ & & & $*$ & & & $\mathrm{~T}$ & & & $\mathrm{P}$ \\
\hline 5 & $128-135$ & $128.05-136.41$ & 80.5 & 69.5 & & & $\dagger$ & & & $*$ & & & $\mathrm{~T}$ & & $\mathrm{~T}$ & $P$ \\
\hline \multicolumn{17}{|c|}{$<2 \mu$ Fraction } \\
\hline 1 & $0-6$ & $0.27-5.37$ & 81.0 & 70.4 & & & 18.7 & 3.9 & 7.9 & 37.9 & 1.6 & 30.0 & - & & & \\
\hline 2 & $51-60$ & $51.19-59.28$ & 75.5 & 61.6 & & & 14.6 & 2.8 & 7.3 & 27.8 & 2.2 & 37.0 & 8.1 & & & \\
\hline 3 & $98-104$ & $99.10-103-90$ & 76.9 & 63.9 & & & 11.6 & - & 9.1 & 27.7 & - & 34.0 & 17.6 & & & \\
\hline 4 & $104-108$ & $104.00-108.00$ & 76.5 & 63.3 & & & 13.6 & - & 5.3 & 34.2 & 3.8 & 32.3 & 10.2 & & & \\
\hline 5 & $128-135$ & $128.05-136.41$ & 83.1 & 73.5 & & & 11.9 & - & 2.2 & 38.0 & 2.6 & 28.8 & 16.7 & & & \\
\hline
\end{tabular}

${ }^{a}$ No entry in column indicates not determined

1st Major Constituents

${ }^{\dagger}$ 2nd Major Constituents

P - Present $(8-2 \%)$

T - Trace $(<2 \%)$ 
TABLE 5

Results of X-Ray Diffraction Analyses from Site $89^{a}$

\begin{tabular}{|c|c|c|c|c|c|c|c|c|c|c|c|c|c|c|c|c|c|}
\hline Core & $\begin{array}{c}\text { Cored } \\
\text { Interval } \\
\text { Below Sea } \\
\text { Floor }(\mathrm{m})\end{array}$ & $\begin{array}{l}\text { Sample Depth } \\
\text { Below Sea } \\
\text { Floor } \\
\text { (m) }\end{array}$ & Diff. & Amor. & Calc. & Dolo. & Quar. & $\mathrm{K}-\mathrm{Fe}$ & Plag. & |Kaol. & Mica & ('hlo. & Mont. & Clin. & Paly. & Pyri. & Gyps. \\
\hline \multicolumn{18}{|c|}{ Bulk Samples } \\
\hline 1 & $0-3$ & $0.07-2.77$ & 78.5 & 42.5 & 35.2 & 1.3 & 19.6 & - & 7.1 & 3.9 & 30.5 & 2.2 & - & & & & \\
\hline 2 & $50-59$ & 51.29 & 71.9 & 35.9 & 45.9 & - & 15.5 & - & 5.9 & 3.5 & 19.8 & 1.2 & 7.2 & & & & \\
\hline 3 & $119-128$ & $119.07-124.32$ & 73.3 & 37.3 & 40.7 & - & 17.8 & - & 7.6 & 3.0 & 22.4 & - & 8.3 & & & & \\
\hline 4 & $220-224$ & $220.02-228.26$ & 68.4 & 32.4 & 56.3 & - & 13.5 & 1.5 & 6.8 & 3.0 & 11.8 & - & 7.1 & & & & \\
\hline 5 & 299-305 & 300.25 & 71.0 & 35.0 & 37.1 & - & 25.6 & - & 10.4 & 1.6 & 23.5 & 1.8 & - & & & & \\
\hline 6 & $376-380$ & $377.24-382.86$ & 69.1 & 33.1 & 31.1 & - & 24.6 & - & 10.0 & 3.2 & 25.8 & 1.1 & 4.2 & & & & \\
\hline \multicolumn{18}{|c|}{ 2-20 $\mu$ Fraction } \\
\hline 1 & $0-3.5$ & $0.07-2.77$ & 74.6 & 60.3 & & & $\dagger$ & & & & * & & & $\mathrm{T}$ & $\mathrm{T}$ & & \\
\hline 2 & $50-59$ & 51.29 & 70.0 & 53.1 & & & * & & & & $\dagger$ & & & $\mathrm{T}$ & $\mathrm{T}$ & & \\
\hline 3 & $119-128$ & $119.07-124.32$ & 76.9 & 63.9 & & & $\dagger$ & & & & * & & & $\mathrm{T}$ & $\mathrm{T}$ & $\mathrm{T}$ & \\
\hline 4 & $220-224$ & $220.02-228.26$ & 72.5 & 57.1 & & & $*$ & & & & $\dagger$ & & & $\mathrm{T}$ & & $\mathbf{P}$ & \\
\hline 5 & $299-305$ & 300.25 & 61.3 & 39.6 & & & $*$ & & & & $\dagger$ & & & & & $\mathrm{T}$ & \\
\hline 6 & $376-380$ & $379.24-382.86$ & 61.8 & 40.3 & & & * & & $\dagger$ & & & & & & & $\mathrm{T}$ & \\
\hline \multicolumn{18}{|c|}{$<2 \mu$ Fraction } \\
\hline 1 & $0-3$ & $0.07-2.77$ & 81.5 & 71.1 & & & 19.7 & & - & - & 38.3 & 4.3 & 27.3 & & 9.0 & - & 1.4 \\
\hline 2 & $50-59$ & 51.29 & 79.9 & 68.7 & & & 10.9 & & 1.3 & 6.2 & 41.6 & 3.0 & 32.3 & & 4.7 & - & - \\
\hline 3 & $119-128$ & $119.07-124.32$ & 81.1 & 70.5 & & & 20.8 & & 2.8 & 5.2 & 39.6 & 4.5 & 27.1 & & - & - & - \\
\hline 4 & $220-229$ & $220.02-228.26$ & 78.3 & 66.1 & & & 11.4 & & - & 2.0 & 21.5 & 2.7 & 42.4 & & 19.8 & - & 1.2 \\
\hline 5 & $299-305$ & 300.25 & 71.4 & 55.2 & & & 31.4 & & 17.9 & 2.3 & 38.6 & 3.5 & 5.8 & & - & - & - \\
\hline 6 & $376-380$ & $379.24-382.86$ & 76.2 & 62.6 & & & 19.3 & & 7.2 & 7.3 & 42.5 & 3.0 & 19.7 & & - & 1.0 & - \\
\hline
\end{tabular}

* No entry in column indicates not determined

1st Major Constituents

†े nd Major Constituents

P - Present $(8-2 \%)$

$\mathrm{T}$ - Trace $(<2 \%)$ 
TABLE 6

Results of X-Ray Diffraction Analyses from Site $90^{\mathrm{a}}$

\begin{tabular}{|c|c|c|c|c|c|c|c|c|c|c|c|c|c|c|c|c|c|c|c|}
\hline Core & $\begin{array}{c}\text { Cored } \\
\text { Interval } \\
\text { Below Sea } \\
\text { Floor }(\mathrm{m})\end{array}$ & $\begin{array}{l}\text { Sample Depth } \\
\text { Below Sea } \\
\text { Floor } \\
\text { (m) }\end{array}$ & Diff. & Amor. & Calc. & Dolo, & Quar. & $\mathrm{K}-\mathrm{Fe}$ & Plag. & Kaol. & Mica & Chlo. & Mont. & Clin. & Amph. & Gyps. & Paly & Pyri. & Phil. \\
\hline \multicolumn{20}{|c|}{ Bulk Samples } \\
\hline 1 & $0-9$ & $0.15-8.27$ & 72.7 & 57.3 & 19.4 & 6.0 & 22.6 & 2.2 & 8.2 & 3.6 & 36.2 & 1.6 & & - & - & - & & & \\
\hline 2 & $70-79$ & $70.60-74.72$ & 76.2 & 62.8 & 21.6 & 2.1 & 22.9 & 1.6 & 7.3 & 2.1 & 39.0 & 3.4 & & - & - & - & & & \\
\hline 3 & $130-140$ & $130.75-133.82$ & 73.9 & 59.3 & 57.1 & - & 14.1 & - & 3.4 & 5.7 & 19.7 & - & & - & - & - & & & \\
\hline 4 & $188-197$ & $189.41-190.72$ & 77.5 & 64.9 & 55.9 & - & 14.0 & - & 4.0 & 4.2 & 21.9 & - & & - & - & - & & & \\
\hline 5 & $236-242$ & $236.20-241.27$ & 79.1 & 67.4 & 15.9 & 1.2 & 30.1 & - & 7.1 & 4.6 & 39.1 & 2.0 & & - & - & - & & & \\
\hline 6 & 293-298 & $293.30-295.27$ & 78.1 & 65.9 & 13.3 & - & 29.2 & - & 7.0 & 5.6 & 42.8 & 2.1 & & - & - & - & & & \\
\hline 7 & 341,348 & $341.49-344.77$ & 75.3 & 61.4 & 22.8 & - & 33.5 & - & 11.9 & 4.2 & 26.4 & 1.2 & & - & - & - & & & \\
\hline 9 & $471-480$ & $471.35-472.07$ & 74.6 & 60.4 & 26.8 & - & 27.8 & - & 10.8 & - & 29.3 & 3.2 & & - & - & 2.0 & & & \\
\hline 10 & $595-597$ & $595-19-595-93$ & 66.2 & 47.1 & 31.4 & 2.0 & 34.6 & - & 18.7 & - & 11.0 & 2.3 & & - & - & - & & & \\
\hline 11 & $675-682$ & $682.71-683.19$ & 71.8 & 56.0 & 26.1 & 1.4 & 27.6 & - & 19.4 & 1.7 & 22.0 & 1.3 & & - & - & - & & & \\
\hline 13 & $763-768$ & $763.10-766.46$ & 67.7 & 49.6 & 12.7 & 2.1 & 24.6 & - & 32.9 & - & 23.4 & 1.1 & & 1.3 & 1.5 & - & & & \\
\hline \multicolumn{20}{|c|}{ 2-20 $\mu$ Fraction } \\
\hline 1 & $0-9$ & $0.15-8.27$ & 69.7 & 52.6 & & $P$ & * & & & & $\dagger$ & & & & & & & & \\
\hline 2 & $70-79$ & $70.60-74.72$ & 70.3 & 53.6 & & & $\dagger$ & & & & $*$ & & & & & & & & \\
\hline 3 & $130-140$ & $130.75-133.82$ & 72.1 & 56.4 & & & * & & & & $\dagger$ & & & & & & & & \\
\hline 4 & $188-197$ & $189.41-190.72$ & 79.4 & 67.8 & & & $\dagger$ & & & & * & & & & & & $\mathrm{T}$ & $\mathrm{T}$ & \\
\hline 5 & $236-242$ & $236.20-241.27$ & 70.7 & 54.3 & & & $\dagger$ & & & & $*$ & & & & & & & $\mathrm{~T}$ & \\
\hline 6 & 293-298 & $293.30-295.27$ & 59.7 & 37.1 & & & * & & $\dagger$ & & & & & & & & $\mathrm{T}$ & $\mathrm{T}$ & \\
\hline 7 & $341-348$ & $341.49-344.77$ & 69.2 & 51.9 & & & $*$ & & & & $\dagger$ & & & & & & & & \\
\hline 9 & $471-480$ & $471.35-472.07$ & 63.6 & 43.0 & & & $*$ & & & & $\dagger$ & & & & & & & $\mathrm{T}$ & \\
\hline 11 & $675-682$ & $682.71-683.19$ & 66.0 & 46.8 & & & * & & $\dagger$ & & & & & & & & & $\mathrm{T}$ & \\
\hline 13 & $763-768$ & $763.10-766.46$ & 70.5 & 53.9 & & & * & & $\dagger$ & & & & & $P$ & & & & $\mathrm{~T}$ & \\
\hline \multicolumn{20}{|c|}{$<2 \mu$ Fraction } \\
\hline 1 & $0-9$ & $0.15-8.27$ & 73.8 & 59.0 & & 1.9 & 20.4 & 4.0 & 7.1 & 3.9 & 50.5 & 3.5 & 8.8 & - & & & & & \\
\hline 2 & $70-79$ & $70.60-74.72$ & 69.5 & 52.4 & & - & 18.5 & - & 7.8 & 4.2 & 61.4 & 3.2 & 4.2 & - & & & & & \\
\hline 3 & $130-140$ & $130.75-133.82$ & 81.2 & 70.6 & & - & 24.2 & - & 5.6 & 12.2 & 45.8 & - & 12.3 & - & & & & & \\
\hline 4 & $188-197$ & $189.41-190.72$ & 75.0 & 60.9 & & - & 28.1 & - & 9.2 & 5.5 & 48.8 & 2.0 & 6.3 & - & & & & & \\
\hline 5 & $236-242$ & $236.20-241.27$ & 76.7 & 63.6 & & - & 23.7 & - & 7.2 & 5.8 & 53.2 & 2.6 & 7.4 & - & & & & & \\
\hline 6 & 293-298 & $293.30-295.27$ & 72.4 & 56.9 & & - & 30.1 & - & 13.1 & 4.2 & 42.7 & 3.0 & 6.6 & - & & & & & \\
\hline 7 & $341-348$ & $341.49-344.77$ & 72.1 & 56.4 & & - & 29.1 & - & 16.2 & 3.9 & 40.9 & 2.8 & 7.1 & - & & & & & \\
\hline 9 & $471-480$ & $471.35-472.07$ & 73.4 & 58.4 & & - & 22.8 & - & 6.3 & 3.2 & 46.5 & 4.0 & 17.2 & - & & & & & \\
\hline 10 & $595-597$ & $595-19-595-93$ & 82.3 & 72.3 & & - & 28.1 & - & - & - & 52.1 & 11.5 & 8.0 & - & & & & & \\
\hline 11 & $675-682$ & $682.71-683.19$ & 77.2 & 64.3 & & - & 21.6 & - & 9.7 & 2.7 & 47.7 & 5.0 & 13.3 & - & & & & & \\
\hline 13 & $763-768$ & $763-10-766.46$ & 71.8 & 55.9 & & - & 16.9 & - & 20.8 & - & 35.3 & 4.4 & 12.5 & 9.1 & & & & & \\
\hline
\end{tabular}

${ }^{\mathrm{a}}$ No entry in column indicates not determined

* 1st Major Constituents

${ }^{\dagger}$ 2nd Major Constituents

$P$ - Present $(8-2 \%)$

T - Trace $(<2 \%)$ 
TABLE 7

Results of X-Ray Diffraction Analyses from Site $91^{\text {a }}$

\begin{tabular}{|c|c|c|c|c|c|c|c|c|c|c|c|c|c|c|c|c|c|c|}
\hline Core & $\begin{array}{c}\text { Cored } \\
\text { Interval } \\
\text { Below Sea } \\
\text { Floor }(\mathrm{m})\end{array}$ & $\begin{array}{l}\text { Sample Depth } \\
\text { Below Sea } \\
\text { Floor } \\
\text { (m) }\end{array}$ & Diff. & Amor. & Calc. & Dolo. & Quar. & $\mathrm{K}-\mathrm{Fe}$ & Plag. & Kaol. & Mica & Chlo. & Mont. & Clin. & Gyps. & Amph. & Pyri. & Phil. \\
\hline \multicolumn{19}{|c|}{ Bulk Samples } \\
\hline 1 & $60-69$ & $61.60-68.27$ & 63.7 & 43.3 & 8.2 & 13.2 & 28.5 & 3.2 & 11.1 & 3.2 & 30.2 & 1.5 & - & - & & 1.0 & & \\
\hline 2 & $123-132$ & $124.60-129.79$ & 75.3 & 61.5 & 7.5 & 7.9 & 28.1 & 2.7 & 12.9 & 3.6 & 33.5 & 2.6 & - & - & & 1.2 & & \\
\hline 3 & $159-168$ & $159.10-167.24$ & 70.8 & 54.3 & 7.1 & 9.0 & 26.8 & 2.3 & 6.4 & 3.6 & 42.5 & 2.1 & - & - & & - & & \\
\hline 4 & $177-186$ & $177.10-185.27$ & 68.1 & 50.2 & 8.1 & 9.3 & 28.8 & 5.2 & 8.2 & 4.9 & 34.1 & 1.0 & - & - & & - & & \\
\hline 5 & $186-195$ & $186.20-194.27$ & 68.7 & 51.1 & 10.0 & 10.0 & 23.9 & 2.6 & 7.5 & 5.2 & 38.1 & 1.7 & - & - & & - & & \\
\hline 6 & $301-310$ & $301.20-308.07$ & 73.5 & 58.7 & 8.0 & 5.4 & 28.3 & - & 9.5 & 5.4 & 41.4 & 2.0 & - & - & & - & & \\
\hline 7 & 407.413 & $407.80-413.77$ & 60.6 & 38.4 & 8.8 & 7.0 & 35.8 & 5.1 & 20.4 & 2.4 & 17.7 & 1.2 & - & - & & 1.6 & & \\
\hline 8 & $490-495$ & $490.66-492.27$ & 60.6 & 38.4 & 23.0 & - & 42.2 & 5.0 & 21.0 & - & 8.7 & - & - & - & & - & & \\
\hline 9 & $530-538$ & $530.15-537.62$ & 62.2 & 40.9 & 27.0 & - & 38.0 & - & 19.8 & - & 13.8 & 1.2 & - & - & & - & & \\
\hline 10 & $652-658$ & $653.60-675.19$ & 68.2 & 50.2 & 25.9 & - & 35.7 & - & 20.3 & 1.1 & 15.8 & 1.2 & - & - & & - & & \\
\hline 11 & $770-779$ & $771.60-775.27$ & 70.1 & 53.2 & 4.4 & 3.0 & 28.3 & 2.1 & 34.3 & 2.1 & 23.1 & 1.0 & - & - & & 1.7 & & \\
\hline 12 & $779-788$ & $782-50-783.00$ & 78.7 & 66.6 & 4.8 & 1.5 & 36.4 & - & 13.0 & 4.5 & 30.9 & 2.2 & 6.7 & - & & - & & \\
\hline 13 & $788-797$ & $789.08-792.17$ & 76.4 & 63.1 & 9.8 & 1.2 & 33.2 & 7.9 & 12.4 & 2.4 & 21.4 & 1.4 & 10.3 & - & & - & & \\
\hline 14 & $797-806$ & $799.30-800.32$ & 70.7 & 54.3 & 18.0 & - & 34.8 & 5.0 & 21.7 & 2.7 & 16.0 & 1.8 & - & - & & - & & \\
\hline 15 & $806-816$ & $806.90-810.57$ & 76.8 & 63.8 & 20.2 & - & 27.3 & - & 11.7 & 2.5 & 24.8 & 2.0 & 11.5 & - & & - & & \\
\hline 16 & $816-825$ & $816.24-819.77$ & 79.5 & 68.0 & 15.8 & 1.4 & 19.2 & - & 13.3 & 3.7 & 31.3 & 2.0 & 11.8 & 1.5 & & - & & \\
\hline 18 & $834-843$ & 842.73 & 73.7 & 58.9 & 3.9 & 1.0 & 16.9 & - & 25.2 & - & 43.6 & 1.9 & - & 7.5 & & - & & \\
\hline 20 & $852-862$ & $854.40-861.07$ & 65.7 & 46.5 & 1.9 & 1.2 & 18.5 & - & 54.5 & - & 18.8 & 1.3 & - & - & & 3.7 & & \\
\hline 22 & $866-872$ & $866.10-868.28$ & 71.3 & 55.1 & 5.2 & 1.1 & 21.3 & - & 34.9 & - & 32.8 & 2.3 & - & - & & 1.4 & & \\
\hline 25 & $890-899$ & $891.68-893.62$ & 63.5 & 42.9 & 1.1 & - & 19.7 & - & 54.2 & - & 23.6 & - & - & - & & 1.4 & & \\
\hline \multicolumn{19}{|c|}{$2-20 \mu$ Fraction } \\
\hline 1 & $60-69$ & $61.60-68.27$ & 70.0 & 53.1 & $\mathrm{P}$ & & $*$ & & & & $\dagger$ & & & & & & & \\
\hline 2 & $123-132$ & $124.60-129.79$ & 62.9 & 42.0 & & & * & & & & $\dagger$ & & & & & & & \\
\hline 3 & $159-168$ & $159.10-167.24$ & 63.0 & 42.1 & & & $*$ & & & & $\dagger$ & & & & & & & \\
\hline 4 & $177-186$ & $177.10-185.27$ & 62.5 & 41.4 & $\mathbf{P}$ & & * & & & & $\dagger$ & & & & & & & \\
\hline 5 & $186-195$ & $186.20-194.27$ & 60.8 & 38.7 & & & * & & & & $\dagger$ & & & & & & & \\
\hline 6 & $301-310$ & $301.20-308.07$ & 60.4 & 38.1 & & & $\dagger$ & & & & $*$ & & & & & & & \\
\hline 7 & $407-413$ & $407.80-413.77$ & 70.8 & 54.4 & & & $\dagger$ & & & & $*$ & & & $\mathrm{~T}$ & & & & \\
\hline 8 & $490-495$ & $490.66-492.27$ & 75.1 & 61.2 & & & $*$ & & & & $\dagger$ & & & & & & $P$ & \\
\hline 9 & $530-538$ & $530.15-537.62$ & 71.9 & 56.0 & & & $\dagger$ & & & & $*$ & & & $\mathrm{~T}$ & & & $P$ & $P$ \\
\hline 10 & $652-658$ & $653.60-657.19$ & 65.1 & 45.5 & & & * & & & & $\dagger$ & & & & & & $\mathrm{T}$ & \\
\hline 11 & $770-779$ & $771.60-775.27$ & 67.7 & 49.6 & & & $\dagger$ & & & & $*$ & & & & & & $\mathrm{~T}$ & \\
\hline 12 & $779-788$ & 782.50 .783 .00 & 63.3 & 42.7 & & & $*$ & & & & $\dagger$ & & & & & & & \\
\hline 13 & $788-797$ & $789.08-792.17$ & 66.2 & 47.1 & & & $*$ & & & & $\dagger$ & & & & & & & \\
\hline 14 & $797-806$ & $799.30-800.32$ & 66.7 & 48.0 & & & $*$ & & & & $\dagger$ & & & & & & & \\
\hline 15 & $806-816$ & $806.90-810.57$ & 68.5 & 50.8 & & & * & & & & $\dagger$ & & & & & & & \\
\hline 18 & $834-843$ & 842.73 & 68.9 & 51.4 & & & & & $\dagger$ & & * & & & A & & & $\mathrm{T}$ & \\
\hline 20 & $852-862$ & $854.40-861.07$ & 89.2 & 83.1 & & & & & & & $*$ & & & $P$ & & & $\mathrm{~T}$ & \\
\hline 22 & $866-872$ & $866.10-868.28$ & 69.6 & 52.6 & & & $\dagger$ & & & & $*$ & & & $\mathrm{P}$ & & & $\mathrm{T}$ & \\
\hline 25 & 890.899 & $891.68-893.62$ & 87.6 & 80.6 & & & $\dagger$ & & * & & & & & & & & $\mathrm{T}$ & \\
\hline
\end{tabular}


TABLE 7 - Continued

\begin{tabular}{|c|c|c|c|c|c|c|c|c|c|c|c|c|c|c|c|c|c|c|}
\hline Core & $\begin{array}{c}\text { Cored } \\
\text { Interval } \\
\text { Below Sea } \\
\text { Floor (m) }\end{array}$ & $\begin{array}{l}\text { Sample Depth } \\
\text { Below Sea } \\
\text { Floor } \\
\text { (m) }\end{array}$ & Diff. & Amor. & Calc. & Dolo. & Quar. & $\mathrm{K}-\mathrm{Fe}$ & Plag. & Kaol. & Mica & Chlo. & Mont. & Clin. & Gyps. & Amph. & Pyri. & Phil. \\
\hline \multicolumn{19}{|c|}{$<2 \mu$ Fraction } \\
\hline 1 & $60-69$ & $61.60-68.27$ & 68.2 & 50.3 & & 2.3 & 24.1 & 5.6 & 10.9 & 2.7 & 45.5 & 3.6 & 3.8 & - & - & 1.6 & & \\
\hline 2 & $123-132$ & $124.60-129.79$ & 69.9 & 52.9 & & - & 21.1 & 7.4 & 11.8 & 3.6 & 45.6 & 3.6 & 5.4 & - & - & 1.5 & & \\
\hline 3 & $159-168$ & $159.10-167.24$ & 71.3 & 55.2 & & - & 15.5 & 1.1 & 3.3 & 5.2 & 64.5 & 3.6 & 6.8 & - & - & - & & \\
\hline 4 & $177-186$ & $177.10-185.27$ & 71.2 & 55.0 & & - & 19.4 & 2.2 & 4.9 & 4.8 & 61.0 & 3.8 & 3.1 & - & - & - & & \\
\hline 5 & $186-195$ & $186.20-194.27$ & 71.2 & 54.9 & & - & 19.7 & 1.7 & 5.3 & 4.7 & 60.7 & 4.5 & 1.9 & - & - & 1.5 & & \\
\hline 6 & $301-310$ & $301.20-308.07$ & 76.5 & 63.3 & & - & 10.2 & 6.1 & 1.9 & 5.1 & 47.2 & 3.1 & 26.1 & - & - & - & & \\
\hline 7 & $407-413$ & $407.80-413.77$ & 75.1 & 61.3 & & - & 10.9 & 5.8 & 6.7 & 6.2 & 46.3 & 4.6 & 19.4 & - & - & - & & \\
\hline 8 & $490-495$ & $490.66-492.27$ & 82.5 & 72.7 & & - & 13.1 & - & - & 6.8 & 41.9 & 3.3 & 33.1 & - & 1.9 & - & & \\
\hline 9 & $530-538$ & $530.15-537.62$ & 81.7 & 71.4 & & - & 19.5 & - & 4.2 & 7.5 & 47.5 & 5.0 & 16.3 & - & - & - & & \\
\hline 10 & $652-658$ & $653.60-657.19$ & 76.0 & 62.5 & & - & 11.9 & - & - & 5.6 & 48.5 & 4.0 & 30.1 & - & - & - & & \\
\hline 11 & $770-779$ & $771.60-775.27$ & 72.7 & 57.3 & & - & 15.9 & - & - & 5.8 & 44.1 & 2.7 & 31.5 & - & - & - & & \\
\hline 12 & $779-788$ & $782.50-783.00$ & 75.1 & 61.1 & & - & 19.2 & - & 4.5 & 7,4 & 49.9 & 3.7 & 15.3 & - & - & - & & \\
\hline 13 & $788-797$ & $789.08-792.17$ & 72.6 & 57.2 & & - & 11.4 & - & - & 6.6 & 40.2 & 2.3 & 39.5 & - & - & - & & \\
\hline 14 & $797-806$ & $799.30-800.32$ & 75.7 & 62.0 & & - & 11.2 & - & - & - & 36.7 & 4.0 & 47.4 & - & - & - & & \\
\hline 15 & $806-816$ & $806.90-810.57$ & 77.2 & 64.4 & & - & 14.8 & - & - & 7.0 & 36.5 & 2.8 & 38.9 & - & - & - & & \\
\hline 16 & $816-825$ & $816.24-819.77$ & 78.3 & 66.1 & & - & 14.6 & - & - & - & 34.0 & 4.9 & 46.5 & - & - & - & & \\
\hline 18 & $834-843$ & 842.73 & 71.9 & 56.1 & & - & 6.3 & - & 4.5 & 1.4 & 40.3 & 4.1 & 38.7 & 4.7 & - & - & & \\
\hline 20 & $852-862$ & $854.40-861.07$ & 70.1 & 53.2 & & - & 6.9 & - & 3.1 & - & 32.8 & 9.6 & 46.9 & - & - & - & & \\
\hline 22 & $866-872$ & $866.10-868.28$ & 75.1 & 61.1 & & - & 10.6 & - & - & - & 40.8 & 7.3 & 41.4 & - & - & - & & \\
\hline 25 & $890-899$ & $891.68-893.62$ & 74.4 & 60.0 & & - & 6.7 & - & 18.6 & - & 33.6 & 11.5 & 29.7 & - & - & - & & \\
\hline
\end{tabular}

${ }^{\mathrm{a}}$ No entry in column indicates not determined

* 1 st Major Constituents

${ }^{2}$ 2nd Major Constituents

P - Present $(8-2 \%)$

T - Trace $(<2 \%)$

A - Abundant $(25-8 \%)$

TABLE 8

Results of X-Ray Diffraction Analyses from Site $92^{\mathrm{a}}$

\begin{tabular}{|c|c|c|c|c|c|c|c|c|c|c|c|c|c|c|c|}
\hline Core & $\begin{array}{c}\text { Cored } \\
\text { Interval } \\
\text { Below Sea } \\
\text { Floor }(\mathrm{m})\end{array}$ & $\begin{array}{l}\text { Sample Depth } \\
\text { Below Sea } \\
\text { Floor } \\
\text { (m) }\end{array}$ & Diff. & Amor.. & Calc. & Dolo. & Quar. & Mica & $\mathrm{K}-\mathrm{Fe}$ & Plag. & Kaol. & Paly. & Mont. & Chlo. & Clin. \\
\hline \multicolumn{16}{|c|}{ Bulk Samples } \\
\hline 2 & $29-38$ & $31.50-35.77$ & 74.3 & 59.8 & 7.2 & 8.5 & 18.0 & 43.2 & 2.5 & 5.1 & 1.7 & - & 8.6 & 5.4 & \\
\hline 3 & $87-96$ & $88.20-93.77$ & 74.3 & 59.8 & 6.5 & 9.8 & 18.2 & 48.7 & 1.5 & 3.7 & 3.2 & - & 5.3 & 3.3 & \\
\hline 4 & $125-134$ & $125.10-131.77$ & 70.9 & 54.5 & 6.8 & 12.0 & 20.3 & 40.8 & 3.7 & 6.4 & 2.8 & - & 3.8 & 3.4 & \\
\hline 5 & $173-182$ & $173.10-181.27$ & 77.7 & 65.1 & 6.7 & 3.8 & 17.8 & 53.4 & 2.0 & 3.9 & 3.5 & - & 5.9 & 2.2 & \\
\hline 6 & $220-229$ & $222.21-222.90$ & 68.6 & 51.0 & 16.0 & 9.3 & 24.8 & 28.1 & 4.0 & 11.7 & 2.8 & - & - & 2.3 & \\
\hline 8 & $262-265$ & 262.84 & 74.6 & 60.3 & 48.5 & 4.2 & 6.6 & 19.1 & - & 2.0 & 1.8 & 3.9 & 12.4 & 1.5 & \\
\hline \multicolumn{16}{|c|}{ 2-20 $\mu$ Fraction } \\
\hline 2 & $29-38$ & $31.50-35.77$ & 71.8 & 55.9 & & $P$ & $\dagger$ & $*$ & & & & & & & \\
\hline 3 & $87-96$ & $88.20-93.77$ & 60.4 & 38.2 & & $\mathbf{P}$ & $\dagger$ & * & & & & & & & \\
\hline 4 & $125-134$ & $125.10-131.77$ & 61.5 & 39.8 & & & $\dagger$ & * & & & & & & & \\
\hline 5 & $173-182$ & $173.10-181.27$ & 80.3 & 69.3 & & & * & $\dagger$ & & & & & & & \\
\hline 6 & $220-229$ & $222.21-222.90$ & 75.0 & 60.9 & & & * & $\dagger$ & & & & & & & $T$ \\
\hline 8 & $262-265$ & 262.84 & 71.1 & 54.8 & & & * & $\dagger$ & & & & & & & $\mathbf{T}$ \\
\hline
\end{tabular}


TABLE 8 - Continued

\begin{tabular}{|c|c|c|c|c|c|c|c|c|c|c|c|c|c|c|}
\hline Core & $\begin{array}{c}\text { Cored } \\
\text { Interval } \\
\text { Below Sea } \\
\text { Floor (m) }\end{array}$ & $\begin{array}{l}\text { Sample Depth } \\
\text { Below Sea } \\
\text { Floot } \\
\text { (m) }\end{array}$ & Diff. & Amor.. & Calc. & Dolo. & Quar. & Mica & $\mathrm{K}-\mathrm{Fe}$ & Plag. & Kaol. & Paly. & Mont. & Chlo. Clin. \\
\hline \multicolumn{15}{|c|}{$<2 \mu$ Fraction } \\
\hline 2 & $29-38$ & $31.50-35.77$ & 68.1 & 50.1 & & & 9.7 & 49.8 & 2.2 & 2.0 & 5.0 & & 27.1 & 4.3 \\
\hline 3 & $87-96$ & $88.20-93.77$ & 72.9 & 57.7 & & & 11.1 & 56.7 & - & 1.7 & 6.4 & & 20.5 & 3.6 \\
\hline 4 & $125-134$ & $125.10-131.77$ & 72.3 & 56.7 & & & 9.8 & 61.2 & - & 2.6 & 6.3 & & 16.3 & 3.8 \\
\hline 5 & $173-182$ & $173.10-181.27$ & 69.5 & 52.4 & & & 10.8 & 53.7 & - & - & 7.0 & & 23.7 & 4.0 \\
\hline 6 & $220-229$ & $222.21-222.90$ & 67.2 & 48.7 & & & 8.5 & 36.3 & 2.1 & 5.4 & 9.7 & & 32.8 & 5.2 \\
\hline 8 & $262-265$ & 262.84 & 74.8 & 60.7 & & & 11.6 & 18.5 & 9.7 & - & 5.0 & & 52.7 & 2.5 \\
\hline
\end{tabular}

${ }^{\mathrm{a}}$ No entry in column indicates not determined

* 1 st Major Constituents

${ }^{\dagger}$ 2nd Major Constituents

P - Present $(8-2 \%)$

T - Trace $(<2 \%)$

TABLE 9

Results of X-Ray Diffraction Analyses from Site $93^{\mathrm{a}}$

\begin{tabular}{|c|c|c|c|c|c|c|c|c|c|c|c|c|c|c|c|c|c|}
\hline Core & $\begin{array}{c}\text { Cored } \\
\text { Interval } \\
\text { Below Sea } \\
\text { Floor }(\mathrm{m})\end{array}$ & $\begin{array}{l}\text { Sample Depth } \\
\text { Below Sea } \\
\text { Floor } \\
\text { (m) }\end{array}$ & Diff. & Amor. & Calc. & Dolo. & Arag. & Quar. & Plag. & Kaol. & Mica & Mont. & Chlo. & Pyri. & Clin. & Paly. & Gyps. \\
\hline \multicolumn{18}{|c|}{ Bulk Samples } \\
\hline 1 & $0-1$ & $\begin{array}{l}0.11 \\
0.76\end{array}$ & $\begin{array}{l}75.7 \\
69.5\end{array}$ & $\begin{array}{l}62.0 \\
52.4\end{array}$ & $\begin{array}{l}57.8 \\
35.1\end{array}$ & $\begin{array}{l}3.0 \\
1.4\end{array}$ & $\overline{44.6}$ & $\begin{array}{l}6.6 \\
4.8\end{array}$ & $\begin{array}{l}1.2 \\
1.6\end{array}$ & $\begin{array}{l}1.2 \\
1.9\end{array}$ & $\begin{array}{r}18.8 \\
8.6\end{array}$ & $\begin{array}{r}10.0 \\
1.8\end{array}$ & $\begin{array}{c}1.2 \\
-\end{array}$ & & & & \\
\hline \multicolumn{18}{|c|}{ 2-20 $\mu$ Fraction } \\
\hline 1 & $0-1.5$ & $\begin{array}{l}0.11 \\
0.76\end{array}$ & $\begin{array}{l}79.3 \\
70.2\end{array}$ & $\begin{array}{l}67.7 \\
53.5\end{array}$ & & & & $\dagger$ & & & * & & & $\begin{array}{l}\mathrm{P} \\
\mathrm{P}\end{array}$ & $\mathrm{T}$ & & \\
\hline \multicolumn{18}{|c|}{$<2 \mu$ Fraction } \\
\hline 1 & $0-1$ & $\begin{array}{l}0.11 \\
0.76\end{array}$ & $\begin{array}{l}74.0 \\
73.6\end{array}$ & $\begin{array}{l}59.3 \\
59.3\end{array}$ & & & & $\begin{array}{l}8.2 \\
5.4\end{array}$ & $\begin{array}{l}1.2 \\
-\end{array}$ & $\begin{array}{l}1.2 \\
-\end{array}$ & $\begin{array}{l}21.6 \\
16.8\end{array}$ & $\begin{array}{l}50.3 \\
56.1\end{array}$ & $\begin{array}{l}2.5 \\
2.8\end{array}$ & & & $\begin{array}{l}13.4 \\
18.9\end{array}$ & $\begin{array}{l}1.0 \\
-\end{array}$ \\
\hline
\end{tabular}

${ }_{*}^{a}$ No entry in column indicates not determined

*1st Major Constituents

† 2 nd Major Constituents

P - Present $(8-2 \%)$

T - Trace $(<2 \%)$ 
TABLE 10

Results of X-Ray Diffraction Analyses from Site $94^{\mathrm{a}}$

\begin{tabular}{|c|c|c|c|c|c|c|c|c|c|c|c|c|c|c|c|c|c|c|}
\hline Core & $\begin{array}{c}\text { Cored } \\
\text { Interval } \\
\text { Below Sea } \\
\text { Floor }(\mathrm{m})\end{array}$ & $\begin{array}{l}\text { Sample Depth } \\
\text { Below Sea } \\
\text { Floot } \\
\text { (m) }\end{array}$ & Ditf. & Amor. & Calc. & Dolo. & Arag. & Quar. & $\mathrm{K}-\mathrm{Fe}$ & Plag. & Kaol. & Mici & Chats. & Mont. & Clin. & Paly. & Pyri. Gyps. & Bari. \\
\hline \multicolumn{19}{|c|}{ Bulk Samples } \\
\hline 1 & $0-2$ & $1.30-2.27$ & 60.9 & 38.8 & 89.8 & - & 8.4 & 1.7 & - & - & - & - & - & - & - & & & \\
\hline 2 & $52-61$ & $52.10-60.27$ & 58.1 & 34.5 & 88.8 & - & 10.0 & 1.2 & - & - & - & - & - & - & - & & & \\
\hline 4 & $129-138$ & $129.10-137.27$ & 55.7 & 30.7 & 99.3 & - & - & 0.7 & - & - & - & - & - & - & - & & & \\
\hline 5 & $168-177$ & $168.20-176.27$ & 56.1 & 31.4 & 97.9 & 1.2 & - & 0.8 & - & - & - & - & - & - & - & & & \\
\hline 6 & $207-216$ & $207.10-215.27$ & 59.1 & 36.1 & 96.6 & 2.1 & - & 1.5 & - & - & - & - & - & - & - & & & \\
\hline 7 & $242-251$ & $243.25-248.77$ & 60.9 & 38.9 & 96.8 & 1.3 & - & 1.8 & - & - & - & - & - & - & - & & & \\
\hline 8 & $251-257$ & $252.60-257.70$ & 60.0 & 37.5 & 92.2 & 5.4 & - & 2.3 & - & - & - & - & - & - & - & & & \\
\hline 9 & $292-301$ & $292.10-300.27$ & 61.0 & 39.1 & 99.2 & - & - & 0.8 & - & - & - & - & - & - & - & & & \\
\hline 10 & $331-336$ & $332.10-334.77$ & 62.7 & 41.7 & 99.0 & - & - & 1.0 & - & - & - & - & - & - & - & & & \\
\hline 11 & $363-369$ & $364.60-369.00$ & 59.0 & 36.0 & 100.0 & - & - & - & - & - & - & - & - & - & - & & & \\
\hline 12 & $369-378$ & $373.60-374.27$ & 61.4 & 39.8 & 100.0 & - & - & - & - & - & - & - & - & - & - & & & \\
\hline 13 & $378-387$ & $381.10-381.77$ & 59.3 & 36.3 & 99.7 & - & - & 0.3 & - & - & - & - & - & - & - & & & \\
\hline 14 & $407-412$ & $410.60-411.27$ & 56.7 & 32.4 & 99.6 & - & - & 0.4 & - & - & - & - & - & - & - & & & \\
\hline 15 & $412-418$ & $417.04-417.83$ & 61.3 & 39.6 & 99.6 & - & - & 0.4 & - & - & - & - & - & - & - & & & \\
\hline 16 & $418-424$ & $421.10-421.77$ & 59.4 & 36.6 & 99.6 & - & - & 0.4 & - & - & - & - & - & - & - & & & \\
\hline 17 & $424-433$ & $430.10-430.77$ & 61.1 & 39.2 & 100.0 & - & - & - & - & - & - & - & - & - & - & & & \\
\hline 18 & $433-442$ & $437.60-438.27$ & 61.5 & 39.8 & 99.6 & - & - & 0.4 & - & - & - & - & - & - & - & & & \\
\hline 19 & $442-451$ & $448.10-448.77$ & 64.7 & 44.9 & 100.0 & - & - & - & - & - & - & - & - & - & - & & & \\
\hline 20 & $451-460$ & $452.60-453.27$ & 62.0 & 40.7 & 100.0 & - & - & - & - & - & - & - & - & - & - & & & \\
\hline 21 & $460-469$ & $461.60-462.27$ & 59.9 & 37.3 & 99.5 & - & - & 0.5 & - & - & - & - & - & - & - & & & \\
\hline 22 & $469-478$ & $473.60-474.27$ & 64.4 & 44.4 & 99.5 & - & - & 0.5 & - & - & - & - & - & - & - & & & \\
\hline 23 & $478-487$ & $479.60-480.27$ & 63.8 & 43.4 & 100.0 & - & - & - & - & - & - & - & - & - & - & & & \\
\hline 24 & $487-496$ & $490.10-490.77$ & 79.8 & 68.5 & 96.6 & - & - & 0.6 & - & 2.7 & - & - & - & - & - & & & \\
\hline 25 & $496-500$ & $499.10-499.77$ & 69.4 & 52.3 & 100.0 & - & - & - & - & - & - & - & - & - & - & & & \\
\hline 26 & $500-507$ & $503.10-503.77$ & 76.4 & 63.2 & 99.3 & - & - & 0.7 & - & - & - & - & - & - & - & & & \\
\hline 28 & $532-540$ & $538.10-538.77$ & 77.6 & 65.0 & 97.2 & 1.5 & - & 1.2 & - & - & - & - & - & - & - & & & \\
\hline 30 & $571-580$ & 571.71 & 76.3 & 63.0 & 100.0 & - & - & - & - & - & - & - & - & - & - & & & \\
\hline 33 & $612-616$ & $613.30-613.72$ & 56.2 & 31.5 & 98.2 & - & - & 0.6 & - & - & - & - & - & - & 1.2 & & & \\
\hline 34 & $616-625$ & 616,73 & 57.9 & 34.2 & 95.6 & - & - & 0.5 & - & - & - & - & - & - & 3.9 & & & \\
\hline 35 & $625-627$ & 626.29 & 61.1 & 39.3 & 92.4 & - & - & 1.4 & 2.2 & 1.1 & - & 2.9 & - & - & - & & & \\
\hline 36 & $627-634$ & $627.00-627.40$ & 58.7 & 35.5 & 91.7 & 1.0 & - & 1.4 & 2.4 & - & - & 3.5 & - & - & - & & & \\
\hline 39 & $643-652$ & 643.96 & 49.8 & 21.5 & 100.0 & - & - & - & - & - & - & - & - & - & - & & & \\
\hline \multicolumn{19}{|c|}{$2-20 \mu$ Fraction } \\
\hline 1 & $0-2$ & $1.3-2.27$ & 72.3 & 56.7 & & $P$ & & * & & & & $\dagger$ & & & $\mathrm{T}$ & & $\mathrm{T}$ & \\
\hline 2 & $52-61$ & $52.1-60.27$ & 78.1 & 65.8 & & & & $\dagger$ & & & & $*$ & & & $\mathrm{~T}$ & & $\mathbf{P}$ & \\
\hline 3 & $100-109$ & $100.75-108.27$ & 84.0 & 74.9 & & $\mathrm{P}$ & & $\dagger$ & & & & * & & & & & $\mathbf{P}$ & \\
\hline 4 & $129-138$ & $129.10-137.27$ & 81.0 & 70.3 & & $\dagger$ & & & & & & * & & & $\mathrm{T}$ & & $P$ & \\
\hline 5 & $168-177$ & $168.20-176.27$ & 74.9 & 60.8 & & * & & $\dagger$ & & & & & & & $\mathrm{T}$ & & $P$ & \\
\hline 6 & $207-216$ & $207.10-215.27$ & 77.7 & 65.2 & & $P$ & & * & & & & $\dagger$ & & & $\mathrm{T}$ & & P & \\
\hline 7 & $242-251$ & $243.25-248.77$ & 85.8 & 77.9 & & & & $*$ & & & & $\dagger$ & & & $\mathrm{T}$ & & $\mathbf{P}$ & \\
\hline 8 & $251-257$ & $258.6-263.77$ & 84.6 & 76.0 & & $\mathrm{P}$ & & * & & & & $\dagger$ & & & $\mathrm{T}$ & $\mathbf{P}$ & $\mathbf{P}$ & \\
\hline 9 & $292-301$ & $292.10-300.27$ & 92.1 & 87.7 & & & & $*$ & & & & $\dagger$ & & & $\mathrm{T}$ & & $P$ & $\mathbf{P}$ \\
\hline 10 & $331-336$ & $332.10-334.77$ & 91.5 & 86.6 & & & & * & & & & $\dagger$ & & & & & $\mathbf{P}$ & A \\
\hline
\end{tabular}


TABLE 10 - Continued

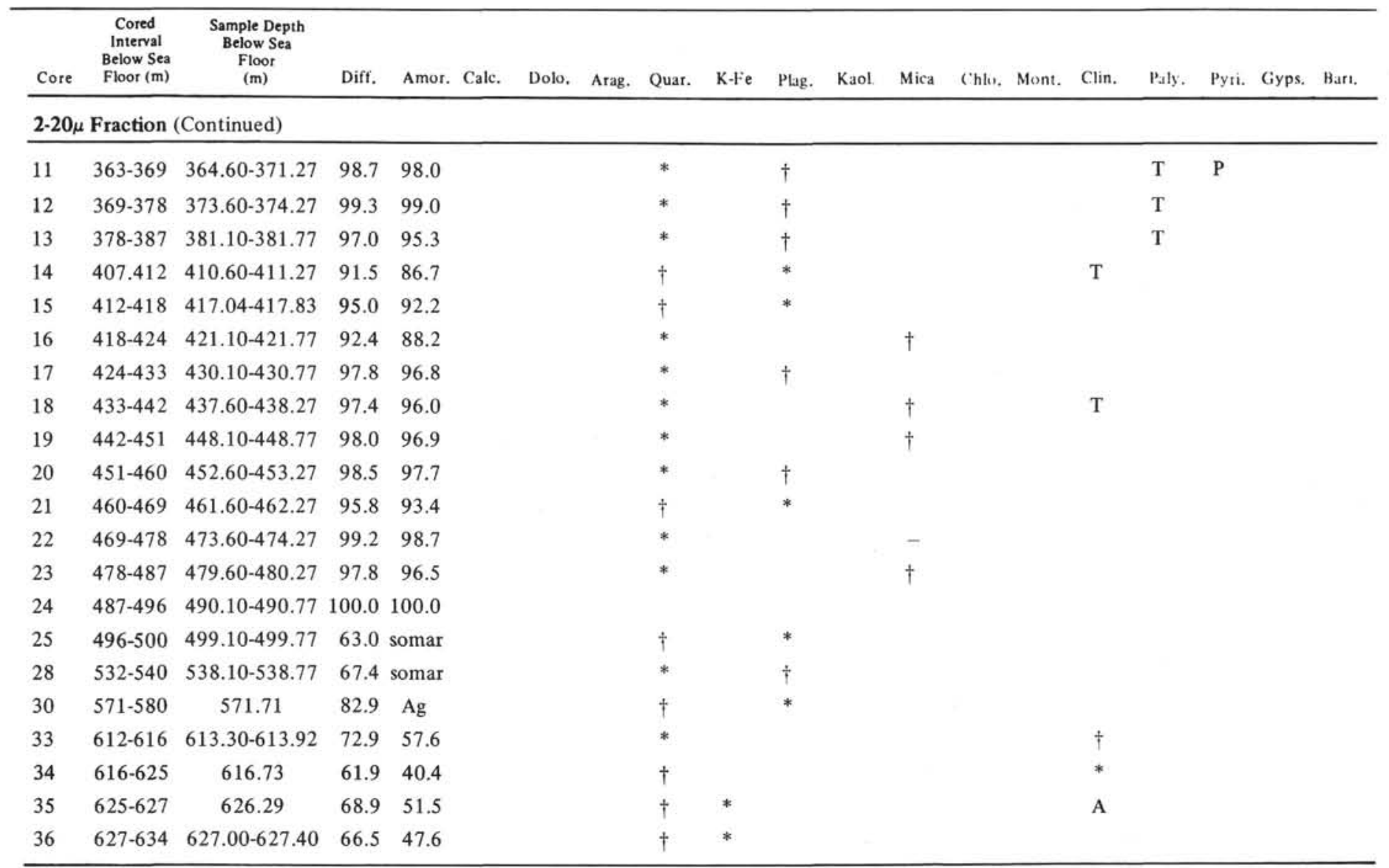

$<2 \mu$ Fraction

\begin{tabular}{|c|c|c|c|c|c|c|c|c|c|c|c|c|c|c|c|}
\hline 1 & $0-2$ & $1.30-2.27$ & 76.9 & 63.8 & 8.4 & 1.4 & 2.6 & 3.2 & 53.5 & 3.2 & 27.6 & - & - & - & - \\
\hline 2 & $52-61$ & $52.10-60.27$ & 77.9 & 65.5 & 11.3 & - & 3.5 & 5.8 & 34.6 & 2.4 & 40.6 & - & - & 1.9 & \\
\hline 3 & $100-109$ & $100.75-108.27$ & 77.4 & 64.8 & 10.8 & 2.8 & 2.0 & 4.5 & 40.2 & 3.4 & 36.3 & - & - & - & - \\
\hline 4 & $129-138$ & $129.10-137.27$ & 75.3 & 61.4 & 11.8 & - & 3.9 & 3.6 & 20.0 & 3.4 & 56.0 & - & - & - & 1.2 \\
\hline 5 & $168-177$ & $168.20-176.27$ & 73.8 & 59.1 & 10.3 & - & 3.4 & 3.7 & 24.5 & 3.5 & 54.1 & - & - & - & - \\
\hline 6 & $207-216$ & $207.10-215.29$ & 74.6 & 60.3 & 11.6 & - & - & 3.8 & 22.2 & 3.5 & 57.7 & - & - & - & 1.2 \\
\hline 7 & $242-251$ & $243.25-258.77$ & 77.4 & 64.7 & 12.8 & - & - & 4.0 & 27.4 & 4.3 & 50.0 & - & - & 1.5 & \\
\hline 8 & $251-257$ & $252.60-257.70$ & 78.6 & 66.5 & 23.2 & - & 8.0 & 2.5 & 32.0 & 3.3 & 28.9 & - & - & 1.9 & - \\
\hline 9 & $292-301$ & $292.10-300.27$ & 85.4 & 77.1 & 16.9 & - & - & 6.3 & 20.6 & 2.5 & 51.8 & - & - & 1.9 & - \\
\hline 10 & $331-336$ & $332.10-334.77$ & 83.0 & 73.5 & 17.5 & - & 6.9 & 7.4 & - & 2.4 & 64.2 & - & - & - & 1.4 \\
\hline 11 & $363-369$ & $364.60-369.00$ & 85.0 & 76.5 & 11.7 & - & 7.5 & 1.4 & - & 2.0 & 77.5 & - & - & - & - \\
\hline 12 & $369-378$ & $373.60-374.27$ & 88.1 & 81.4 & 12.0 & 10.7 & 5.6 & 4.6 & 10.8 & 1.7 & 54.6 & - & - & - & - \\
\hline 13 & $378-387$ & $381.10-381.77$ & 91.7 & 87.1 & 14.0 & - & - & 6.6 & - & 1.5 & 77.6 & - & - & - & - \\
\hline 14 & $407-412$ & $410.60-411.27$ & 78.8 & 66.8 & 9.6 & - & 4.4 & 5.5 & 12.2 & 1.9 & 66.3 & - & - & - & - \\
\hline 15 & $412-418$ & $417.04-417.83$ & 83.1 & 73.6 & 8.8 & - & 3.3 & 5.2 & 12.2 & 1.2 & 58.5 & - & 10.8 & - & - \\
\hline 16 & $418-424$ & $421.10-421.77$ & 81.4 & 70.9 & 11.1 & - & - & 2.6 & 14.9 & 2.3 & 68.5 & - & - & - & - \\
\hline 17 & $424-433$ & $430.10-430.77$ & 83.5 & 74.3 & 9.1 & - & - & 2.0 & 19.0 & 2.0 & 65.4 & - & - & - & 2.4 \\
\hline 18 & $433-442$ & $437.60-438.27$ & 86.3 & 79.4 & 12.1 & - & - & 5.0 & - & 3.0 & 76.1 & - & - & - & 3.7 \\
\hline 19 & $442-451$ & $448.10-448.77$ & 90.9 & 85.8 & 16.4 & - & - & 8.5 & - & 1.0 & 74.0 & - & - & - & - \\
\hline 20 & $451-460$ & $452.60-453.27$ & 91.2 & 86.2 & 9.5 & - & - & 6.4 & 17.7 & - & 66.2 & - & - & - & - \\
\hline 21 & $460-469$ & $461.60-462.27$ & 80.6 & 69.6 & 8.9 & - & - & 3.7 & 12.3 & 2.5 & 70.8 & - & - & - & 1.4 \\
\hline 22 & $469-478$ & $473.60-474.27$ & 90.9 & 85.9 & 20.7 & - & - & 6.4 & 14.4 & - & 58.5 & - & - & - & - \\
\hline
\end{tabular}


TABLE 10 - Continued

\begin{tabular}{|c|c|c|c|c|c|c|c|c|c|c|c|c|c|c|c|c|c|c|c|}
\hline Core & $\begin{array}{c}\text { Cored } \\
\text { Interval } \\
\text { Below Sea } \\
\text { Floor }(\mathrm{m})\end{array}$ & $\begin{array}{l}\text { Sample Depth } \\
\text { Below Sea } \\
\text { Floor } \\
\text { (m) }\end{array}$ & Diff. & Amor. & Calc. & Dolo. & Arag. & Quar. & $\mathrm{K} \cdot \mathrm{Fe}$ & Plag. & Kaol. & Mica & Chlo. & Mont. & Clin. & Paly. & Pyri. & Gyps. & Bari. \\
\hline \multicolumn{20}{|c|}{$<2 \mu$ Fraction (Continued) } \\
\hline 23 & $478-487$ & $479.60-480.27$ & 96.7 & 94.8 & & & & 9.3 & - & - & 4.0 & 26.3 & 2.0 & 58.4 & - & - & - & - & \\
\hline 24 & $489-496$ & $490.10-490.77$ & 98.4 & 97.5 & & & & 20.8 & - & - & - & - & - & 79.1 & - & - & - & - & \\
\hline 25 & $496-500$ & $499.10-499.77$ & 94.1 & 90.8 & & & & 20.7 & - & - & 8.0 & - & - & 60.9 & - & 10.0 & - & - & \\
\hline 26 & $500-507$ & $503.10-503.77$ & 98.2 & 97.2 & & & & 10.0 & - & - & - & - & - & 28.4 & - & 61.5 & - & - & \\
\hline 28 & $532-540$ & $538.10-538.77$ & 98.1 & 97.1 & & & & 100.0 & - & - & - & - & - & - & - & - & - & - & \\
\hline 30 & $571-580$ & 571.71 & 98.0 & 96.8 & & & & 6.8 & - & - & - & - & - & 18.2 & - & 74.8 & - & - & \\
\hline 33 & $612-616$ & $613.30-613.92$ & 76.8 & 63.8 & & & & 10.2 & - & 4.8 & - & 22.1 & 4.3 & 53.4 & 5.2 & - & - & - & \\
\hline 35 & $625-627$ & 626.29 & 76.3 & 63.0 & & & & 17.3 & 22.5 & 5.1 & - & 32.2 & 4.0 & 11.7 & 7.1 & - & - & - & \\
\hline 36 & $627-634$ & $627.00-627.40$ & 70.5 & 53.9 & & & & 24.4 & 31.1 & 2.0 & - & 33.3 & 4.9 & 4.3 & - & - & - & - & \\
\hline
\end{tabular}

${ }_{*}^{a}$ No entry in column indicates not determined

* 1 st Major Constituents

$\dagger$ 2nd Major Constituents

P - Present $(8-2 \%)$

T - Trace $(<2 \%)$

A - Abundant (25-8\%)

TABLE 11

Results of X-Ray Diffraction Analyses from Site $95^{\text {a }}$

\begin{tabular}{|c|c|c|c|c|c|c|c|c|c|c|c|c|c|c|c|c|c|}
\hline Core & $\begin{array}{c}\text { Cored } \\
\text { Interval } \\
\text { Below Sea } \\
\text { Floor (m) }\end{array}$ & $\begin{array}{l}\text { Sample Depth } \\
\text { Below Sea } \\
\text { Floor } \\
\text { (m) }\end{array}$ & Ditt. & Amor. & Calc. & Dolo. & Arag. & Quar. & K-lie & Plag. & Kaol. & Mica & ('hles. Mont. & Paly. & Clin. & Pyri. Gyps. & Cris. \\
\hline \multicolumn{18}{|c|}{ Bulk Samples } \\
\hline 1 & $0-7$ & $0.75-6.12$ & 69.0 & 51.5 & 66.8 & 2.8 & 7.5 & 6.5 & & 1.8 & 2.3 & 11.9 & & & - & & \\
\hline 2 & $82-91$ & $82.10-90.27$ & 54.3 & 28.7 & 99.6 & - & - & 0.4 & & - & - & - & & & - & & \\
\hline 3 & $121-130$ & $121.10-129.27$ & 58.4 & 35.1 & 99.5 & - & - & 0.5 & & - & - & - & & & - & & \\
\hline 4 & $159-168$ & $160.60-164.27$ & 59.7 & 37.0 & 99.6 & - & - & 0.4 & & - & - & - & & & - & & \\
\hline 5 & $198-207$ & $198.05-103.27$ & 58.0 & 34.3 & 99.7 & - & - & 0.3 & & - & - & - & & & - & & \\
\hline 6 & $236-245$ & $236.10-244.27$ & 55.5 & 30.4 & 100.0 & - & - & - & & - & - & - & & & - & & \\
\hline 7 & $274-283$ & $274.10-282.27$ & 62.2 & 40.9 & 99.6 & - & - & 0.4 & & - & - & - & & & - & & \\
\hline 8 & $332-341$ & $332.10-340.27$ & 67.4 & 49.0 & 100.0 & - & - & - & & - & - & - & & & - & & \\
\hline 10 & $363-371$ & $363.10-363.77$ & 52.7 & 26.0 & 98.3 & - & - & - & & - & - & - & & & 1.7 & & \\
\hline 11 & $377-386$ & $378.18-378.60$ & 58.1 & 34.5 & 96.3 & - & - & 0.9 & & - & - & - & & & 2.8 & & \\
\hline 12 & $386-395$ & $390.60-391.27$ & 59.3 & 36.4 & 94.3 & - & - & 0.6 & & 2.3 & - & - & & & 2.8 & & \\
\hline 13 & $395-400$ & $395.70-396.15$ & 55.2 & 30.0 & 98.8 & - & - & 0.6 & & - & - & - & & & - & & \\
\hline 14 & $400-408$ & 40.0 .57 & 55.2 & 29.9 & 97.0 & - & - & - & & - & - & - & & & 3.0 & & \\
\hline 15 & $408-417$ & $415.60-416.27$ & 53.3 & 27.1 & 99.6 & - & - & 0.4 & & - & - & - & & & - & & \\
\hline 16 & $417-426$ & $424.60-425.27$ & 50.9 & 23.3 & 100.0 & - & - & - & & - & - & - & & & - & & \\
\hline 17 & $426-435$ & $433.60-434.27$ & 50.2 & 22.2 & 100.0 & - & - & - & & - & - & - & & & - & & \\
\hline
\end{tabular}


TABLE 11 - Continued

\begin{tabular}{|c|c|c|c|c|c|c|c|c|c|c|c|c|c|c|c|c|c|c|c|}
\hline Core & $\begin{array}{c}\text { Cored } \\
\text { Interval } \\
\text { Below Sea } \\
\text { Floor (m) }\end{array}$ & $\begin{array}{l}\text { Sample Depth } \\
\text { Below Sea } \\
\text { Floot } \\
\text { (m) }\end{array}$ & Diff. & Amor. & Calc. & Dolo. & Arag. & Quar. & K-Fe & Plag. & Kaol. & Mica & Chitu. & Mont. & Paly. & Clin. & Pyri. & Gyps. & Cris. \\
\hline \multicolumn{20}{|c|}{$2-20 \mu$ Fraction } \\
\hline 1 & $0-7$ & $0.75-6.12$ & 66.3 & 47.3 & & A & & $*$ & & & & $\dagger$ & & & & & & & \\
\hline 2 & $82-91$ & $82.10-90.27$ & 93.0 & 89.1 & & & & $*$ & & & & $\dagger$ & & & $\mathbf{T}$ & & & & \\
\hline 3 & $121-130$ & $121.10-129.27$ & 92.4 & 88.1 & & & & * & & & & $\dagger$ & & & $\mathrm{T}$ & & & & A \\
\hline 4 & $159-168$ & $160.60-164.27$ & 95.4 & 92.8 & & & & * & & $\dagger$ & & & & & & & & & $\mathrm{T}$ \\
\hline 5 & $198-207$ & $198.05-203.27$ & 92.0 & 87.5 & & & & * & & & & $\dagger$ & & & $\mathrm{T}$ & $\mathrm{T}$ & & & $\mathrm{T}$ \\
\hline 6 & $236-245$ & $236.10-244.27$ & 93.7 & 90.1 & & & & $\dagger$ & * & & & & & & & & & & $\mathrm{T}$ \\
\hline 7 & $274-283$ & $274.10-282.27$ & 97.4 & 96.0 & & & & * & $\dagger$ & & & & & & & & & & $\mathrm{T}$ \\
\hline 8 & $332-341$ & $332.10-340.27$ & 96.5 & 94.5 & & & & $\dagger$ & * & & & & & & & & & & \\
\hline 10 & $363-371$ & $363.10-363.77$ & 63.1 & 42.4 & & & & $\dagger$ & & & & & & & & * & & & \\
\hline 11 & $377-386$ & $378.10-378.60$ & 66.1 & 47.1 & & & & & $\dagger$ & & & & & & & * & & & \\
\hline 13 & $397-400$ & $395.70-396.15$ & 63.6 & 43.2 & & & & * & $\dagger$ & & & & & & & & & & \\
\hline 14 & $400-408$ & 400.57 & 60.9 & 38.9 & & & & & $\dagger$ & & & & & & & * & & & \\
\hline 15 & $408-417$ & $415.60-416.27$ & 61.6 & 40.0 & & & & * & & $\dagger$ & & & & & & & $\mathbf{P}$ & & \\
\hline 17 & $426-438$ & $433.60-434.27$ & 62.3 & 41.1 & & & & $\dagger$ & * & & & & & & & & $\mathbf{P}$ & & \\
\hline \multicolumn{20}{|c|}{$<2 \mu$ Fraction } \\
\hline 1 & $0-7$ & $0.75-6.12$ & 77.1 & 64.2 & & & & 12.8 & 2.6 & 4.5 & 6.3 & 40.0 & 3.1 & 30.6 & - & - & - & - & \\
\hline 2 & $82-91$ & $82.10-90.27$ & 83.2 & 73.7 & & & & 13.1 & - & - & 2.6 & 21.4 & 1.5 & 61.4 & - & - & - & - & \\
\hline 3 & $121-130$ & $121.10-129.27$ & 84.9 & 76.4 & & & & 9.7 & - & 4.3 & 2.2 & 10.2 & 2.1 & 71.3 & - & - & - & - & \\
\hline 4 & $159-168$ & $160.60-164.27$ & 84.3 & 75.5 & & & & 8.3 & 2.8 & 2.6 & 4.9 & 13.0 & 2.0 & 66.5 & - & - & - & - & \\
\hline 5 & $198-207$ & $198.05-203.27$ & 85.7 & 77.7 & & & & 5.7 & 1.5 & 1.9 & 3.8 & 25.6 & 1.7 & 60.0 & - & - & - & - & \\
\hline 6 & $236-245$ & $236.10-244.27$ & 82.1 & 72.1 & & & & 12.8 & - & 3.4 & 4.4 & 11.9 & 2.4 & 64.7 & - & - & - & - & \\
\hline 7 & $274-283$ & $274.10-282.27$ & 83.5 & 74.2 & & & & 9.5 & - & - & 6.0 & - & 1.0 & 83.4 & - & - & - & - & \\
\hline 8 & $332-341$ & $332.10-340.27$ & 89.3 & 83.3 & & & & 7.9 & 3.1 & - & 5.0 & - & 1.5 & 44.6 & 37.6 & - & - & - & \\
\hline 10 & $363-371$ & $363.10-363.77$ & 77.5 & 64.8 & & & & 6.3 & - & - & 1.5 & 20.4 & 2.0 & 41.5 & 16.1 & 11.9 & - & - & \\
\hline 11 & $377-386$ & $378.18-378.60$ & 76.9 & 63.9 & & & & 7.4 & 6.7 & - & 1.0 & 22.2 & 2.5 & 36.8 & 13.4 & 10.0 & - & - & \\
\hline 12 & $386-395$ & $390.60-391.27$ & 75.5 & 61.7 & & & & 6.4 & 8.0 & - & 1.3 & 13.9 & 2.2 & 39.5 & 25.6 & 3.4 & - & - & \\
\hline 13 & $395-400$ & $395.70-396.15$ & 74.9 & 60.8 & & & & 7.1 & 3.4 & - & 2.2 & 12.4 & 2.2 & 45.7 & 22.6 & 4.1 & - & - & \\
\hline 14 & $400-408$ & 400.57 & 79.2 & 67.5 & & & & 2.5 & 4.6 & - & - & 13.7 & 1.4 & 38.3 & 23.6 & 15.9 & - & - & \\
\hline 15 & $408-417$ & $415.60-416.27$ & 68.2 & 50.3 & & & & 1.5 & 2.3 & - & - & 7.8 & 1.5 & 76.5 & 9.4 & - & - & 1.0 & \\
\hline 16 & $417-426$ & $424.60-425.27$ & 86.4 & 78.7 & & & & 13.2 & 18.7 & - & - & 31.6 & 4.6 & 6.3 & 21.9 & - & 3.7 & - & \\
\hline 17 & $426-435$ & $433.60-434.27$ & 86.0 & 78.1 & & & & 5.9 & 4.5 & - & - & 34.3 & 4.3 & 24.1 & 25.9 & - & - & 1.0 & \\
\hline
\end{tabular}

${ }^{a}$ No entry in column indicates not determined

*1 1 st Major Constituents

$\dagger$ 2nd Major Constituents

P - Present (8-2\%)

T - Trace $(<2 \%)$

A - Abundant (25-8\%) 
TABLE 12

Results of X-Ray Diffraction Analyses from Site $96^{\mathrm{a}}$

\begin{tabular}{|c|c|c|c|c|c|c|c|c|c|c|c|c|c|c|c|}
\hline Core & $\begin{array}{c}\text { Cored } \\
\text { Interval } \\
\text { Below Sea } \\
\text { Floor }(\mathrm{m})\end{array}$ & $\begin{array}{l}\text { Sample Depth } \\
\text { Below Sea } \\
\text { Floor } \\
\text { (m) }\end{array}$ & Diff. & Amor & Dolo. & Quar. & Calc. & Mica & K-Fe & Plag. & Kaol. & Clin. & Mont. & Chlo. & Paly. \\
\hline \multicolumn{16}{|c|}{ Bulk Samples } \\
\hline 1 & $101-110$ & $102-60-109-27$ & 82.2 & 72.1 & 3.0 & 15.1 & 13.4 & 33.1 & 1.7 & 3.0 & 3.4 & - & 24.8 & 2.3 & \\
\hline 2 & $199-208$ & $199.10-207.27$ & 57.5 & 33.6 & - & 0.8 & 99.2 & - & - & - & - & - & - & - & \\
\hline 3 & $301-310$ & $301.15-309.27$ & 64.2 & 44.0 & - & 0.9 & 99.1 & - & - & - & - & - & - & - & \\
\hline 5 & $329-332$ & $329.20-329.83$ & 63.5 & 43.0 & - & 0.8 & 95.1 & - & 1.2 & - & - & 2.8 & - & - & \\
\hline \multicolumn{16}{|c|}{ 2-20 $\mu$ Fraction } \\
\hline 1 & $101-110$ & $102.60-109.27$ & 64.8 & 45.1 & & $\dagger$ & & * & & & & $\mathrm{T}$ & & & \\
\hline 2 & $199-208$ & $119.10-207.27$ & 84.1 & 75.1 & & * & & $\dagger$ & & & & A & & & \\
\hline 3 & $301-310$ & $301.15-309.27$ & 93.3 & 89.6 & & * & & $\dagger$ & & & & $\mathrm{T}$ & & & \\
\hline 5 & $329-332$ & $329.20-329.83$ & 76.1 & 62.7 & & $\dagger$ & & & & & & * & & & \\
\hline \multicolumn{16}{|c|}{$<2 \mu$ Fraction } \\
\hline 1 & $101-110$ & $102.60-109.27$ & 71.4 & 55.4 & & 10.9 & & 33.9 & & 2.6 & 5.7 & - & 44.4 & 2.3 & - \\
\hline 2 & $199-208$ & $199.10-207.27$ & 81.2 & 70.6 & & 12.0 & & 13.2 & & - & - & 3.7 & 57.7 & 2.4 & 10.6 \\
\hline 3 & $301-310$ & $301.15-309.27$ & 83.6 & 74.3 & & 11.0 & & 18.6 & & 2.7 & - & - & 53.3 & 3.9 & 10.5 \\
\hline 5 & $329-332$ & $329.20-329.83$ & 78.3 & 66.1 & & 6.1 & & 21.3 & & - & 1.2 & - & 55.8 & 2.7 & 12.1 \\
\hline
\end{tabular}

${ }^{\mathrm{a}}$ No entry in column indicates not determined

* 1st Major Constituents

$\dagger$ 2nd Major Constituents

P - Present $(8-2 \%)$

T - Trace $(<2 \%)$

A - Abundant (25-8\%)

TABLE 13

Results of X Ray Diffraction Analyses from Site $97^{\mathrm{a}}$

\begin{tabular}{|c|c|c|c|c|c|c|c|c|c|c|c|c|c|c|c|c|c|c|}
\hline Core & $\begin{array}{c}\text { Cored } \\
\text { Interval } \\
\text { Below Sea } \\
\text { Floot }(\mathrm{m})\end{array}$ & $\begin{array}{l}\text { Sample Depth } \\
\text { Below Sea } \\
\text { Floor } \\
\text { (m) }\end{array}$ & Diff. & Amor. & Calc. & Dolo. & Arag. & Quar. & $\mathrm{K}-\mathrm{Fe}$ & Plag. & Kaol. & Mica & Chlo. & Mont. & Paly. & Clin. & Pyri. & Gyps. \\
\hline \multicolumn{19}{|c|}{ Bulk Samples } \\
\hline 1 & $0-5$ & $\begin{array}{l}0.67-1.27 \\
2.10-4.27\end{array}$ & $\begin{array}{l}74.4 \\
63.5\end{array}$ & $\begin{array}{l}60.0 \\
43.0\end{array}$ & $\begin{array}{l}31.3 \\
89.2\end{array}$ & $\begin{array}{l}2.0 \\
-\end{array}$ & $\begin{array}{r}16.7 \\
3.3\end{array}$ & $\begin{array}{r}10.1 \\
2.1\end{array}$ & - & $\begin{array}{l}2.2 \\
-\end{array}$ & $\begin{array}{l}4.2 \\
1.3\end{array}$ & $\begin{array}{r}22.6 \\
4.1\end{array}$ & & $\begin{array}{c}10.5 \\
-\end{array}$ & & - & & \\
\hline 2 & $105-110$ & $105.20-110.27$ & 69.2 & 51.9 & 87.2 & - & - & 5.2 & - & 1.7 & - & 6.0 & & - & & - & & \\
\hline 3 & $142-145$ & $143.60-145.77$ & 72.7 & 57.4 & 80.4 & - & - & 6.7 & 2.3 & 3.4 & - & 7.1 & & - & & - & & \\
\hline 4 & $200-207$ & $202.50-206.77$ & 61.3 & 39.6 & 96.1 & - & - & 1.3 & - & - & - & - & & - & & 2.6 & & \\
\hline 5 & $250-253$ & 251.25 & 60.6 & 38.4 & 93.9 & - & - & 0.7 & - & - & - & - & & 5.7 & & - & & \\
\hline 6 & $294-298$ & 294.86 & 67.6 & 49.4 & 78.1 & 11.2 & - & 0.8 & - & - & - & 9.7 & & - & & - & & \\
\hline 7 & $305-308$ & $306.10-306.77$ & 63.8 & 43.4 & 89.8 & 4.2 & - & - & - & - & - & 5.6 & & - & & - & & \\
\hline 8 & $308-313$ & $308.38-311.90$ & 72.0 & 56.2 & 64.1 & 3.9 & - & 3.6 & 1.1 & 2.8 & - & 20.2 & & 4.2 & & - & & \\
\hline 11 & $330-333$ & 330.30 & 54.4 & 28.8 & 100.0 & - & - & - & - & - & - & - & & - & & - & & \\
\hline 12 & $333-337$ & 333.64 & 57.8 & 34.0 & 98.8 & - & - & 1.2 & - & - & - & - & & - & & - & & \\
\hline
\end{tabular}


TABLE 13 - Continued

\begin{tabular}{|c|c|c|c|c|c|c|c|c|c|c|c|c|c|c|c|c|c|c|}
\hline Core & $\begin{array}{c}\text { Cored } \\
\text { Interval } \\
\text { Below Sea } \\
\text { Floor (m) }\end{array}$ & $\begin{array}{l}\text { Sample Depth } \\
\text { Below Sea } \\
\text { Floor } \\
\text { (m) }\end{array}$ & Diff. & Amor. & Calc. & Dolo. & Arag. & Quar. & $\mathrm{K}-\mathrm{Fe}$ & Plag. & Kaol. & Mica & Chlo. & - Mont. & Paly. & Clin. & Pyri & Gyps. \\
\hline \multicolumn{19}{|c|}{$2-20 \mu$ Fraction } \\
\hline 1 & $0-5$ & $\begin{array}{l}0.67-1.27 \\
2.10-4.27\end{array}$ & $\begin{array}{l}70.3 \\
75.0\end{array}$ & $\begin{array}{l}53.6 \\
60.9\end{array}$ & & & & $\dagger$ & & & & $\stackrel{*}{\dagger}$ & & & & & & \\
\hline 2 & $105-110$ & $105.20-110.27$ & 69.9 & 53.0 & & & & * & & & & $\dagger$ & & & & $P$ & & \\
\hline 3 & $142-145$ & $143.60-145.77$ & 62.8 & 41.8 & & & & * & & & & $\dagger$ & & & & $P$ & $P$ & \\
\hline 4 & $200-207$ & $202.50-206.77$ & 84.1 & 75.2 & & & & $\dagger$ & & & & & & & & $*$ & & \\
\hline 5 & $250-253$ & 251.25 & 69.4 & 52.3 & & & & * & & & & $\dagger$ & & & & A & & \\
\hline 6 & $294-298$ & 294.86 & 69.6 & 52.5 & & & & * & & & & $\dagger$ & & & & & P & \\
\hline 7 & $305-308$ & $306.10-306.77$ & 59.0 & 36.0 & & & & $\dagger$ & & & & $*$ & & & & & $P$ & \\
\hline 8 & $308-313$ & $308.38-311.90$ & 69.8 & 52.8 & & & & $\dagger$ & & & & * & & & & & $P$ & \\
\hline 12 & $333-337$ & 333.64 & 79.2 & 67.6 & & & & $*$ & & & & $\dagger$ & & & & & & \\
\hline \multicolumn{19}{|c|}{$<2 \mu$ Fraction } \\
\hline 1 & $0-5$ & $\begin{array}{l}0.67-1.27 \\
2.10-4.27\end{array}$ & $\begin{array}{l}72.7 \\
77.9\end{array}$ & $\begin{array}{l}57.3 \\
65.4\end{array}$ & & & & $\begin{array}{r}8.0 \\
13.0\end{array}$ & & $\begin{array}{l}1.1 \\
3.1\end{array}$ & $\begin{array}{r}5.9 \\
16.0\end{array}$ & $\begin{array}{l}26.1 \\
42.6\end{array}$ & $\begin{array}{l}2.6 \\
1.4\end{array}$ & $\begin{array}{l}56.4 \\
23.8\end{array}$ & - & $\begin{array}{l}- \\
-\end{array}$ & - & - \\
\hline 2 & $105-110$ & $105.20-110.27$ & 72.0 & 56.3 & & & & 12.9 & & - & 4.5 & 29.8 & 1.9 & 49.1 & - & 1.2 & - & - \\
\hline 3 & $142-145$ & $143.60-145.77$ & 80.8 & 70.0 & & & & 11.7 & & - & 5.1 & 33.4 & 2.1 & 43.5 & - & 1.4 & 1.4 & 1.0 \\
\hline 4 & $200-207$ & $202.50-206.77$ & 78.3 & 66.1 & & & & 10.3 & & - & - & 29.4 & 2.0 & 48.7 & - & 8.4 & - & 1.2 \\
\hline 5 & $250-253$ & 251.25 & 73.6 & 58.8 & & & & 2.0 & & - & 1.0 & 11.0 & - & 77.6 & 7.2 & - & - & 1.0 \\
\hline 6 & $294-298$ & 294.86 & 76.3 & 63.0 & & & & 3.7 & & - & - & 63.4 & - & 32.6 & - & - & - & - \\
\hline 7 & $305-308$ & $306.10-306.77$ & 78.6 & 66.5 & & & & 2.3 & & - & - & 60.7 & - & 36.3 & - & - & - & - \\
\hline 8 & $308-313$ & $308.38-311.90$ & 72.7 & 57.4 & & & & - & & - & - & 48.8 & - & 51.2 & - & - & - & - \\
\hline 12 & $333-337$ & 333.64 & 84.6 & 75.9 & & & & 3.9 & & - & - & - & - & 66.7 & 28.5 & - & - & 1.0 \\
\hline
\end{tabular}

${ }^{a}$ No entry in column indicates not determined

* 1 st Major Constituents

$\dagger_{2 \text { 2nd Major Constituents }}$

P - Present $(8-2 \%)$

T - Trace $(<2 \%)$

A - Abundant $(25-8 \%)$ 
TABLE 14

Sediment Samples from Leg 10 Submitted for X-Ray Diffraction Analyses

\begin{tabular}{|c|c|c|c|c|}
\hline Hole & Core & Section & $\begin{array}{l}\text { Depth in } \\
\text { Section } \\
(\mathrm{cm})\end{array}$ & $\begin{array}{l}\text { Depth Below } \\
\text { Sea Floor } \\
\text { (m) }\end{array}$ \\
\hline \multirow[t]{31}{*}{85} & \multirow[t]{10}{*}{1} & \multirow[t]{2}{*}{1} & $5.0-7.5$ & $19.05-27.51$ \\
\hline & & & $71.0-73.5$ & $19.05-27.51$ \\
\hline & & \multirow[t]{2}{*}{2} & $14.0-16.5$ & $19.05-27.51$ \\
\hline & & & $74.0-76.5$ & $19.05-17.51$ \\
\hline & & \multirow[t]{2}{*}{3} & $10.0-12.5$ & $19.05-27.51$ \\
\hline & & & $70.0-72.5$ & $19.05-27.51$ \\
\hline & & 4 & $19.0-21.5$ & $19.05-27.51$ \\
\hline & & \multirow[t]{2}{*}{5} & $17.0-19.5$ & $19.05-27.51$ \\
\hline & & & $85.0-87.5$ & $19.05-27.51$ \\
\hline & & 6 & $24.0-26.5$ & $19.05-27.51$ \\
\hline & \multirow[t]{6}{*}{2} & \multirow[t]{2}{*}{1} & 8.0-10.5 & $48.08-51.77$ \\
\hline & & & $77.0-79.5$ & $48.08-51.77$ \\
\hline & & \multirow[t]{2}{*}{2} & $32.0-34.5$ & $48.08-51.77$ \\
\hline & & & $80.0-82.5$ & $48.08-51.77$ \\
\hline & & \multirow[t]{2}{*}{3} & $10.0-12.5$ & $48.08-51.77$ \\
\hline & & & $75.0-77.5$ & $48.08-51.77$ \\
\hline & \multirow[t]{10}{*}{3} & \multirow[t]{2}{*}{1} & $12.0-15.5$ & $99.12-107.72$ \\
\hline & & & $84.0-86.5$ & $99.12-107.72$ \\
\hline & & \multirow[t]{2}{*}{2} & $9.5-13.0$ & $99.12-107.72$ \\
\hline & & & $76.5-80.0$ & $99.12-107.72$ \\
\hline & & \multirow[t]{2}{*}{3} & $9.0-11.0$ & $99.12-107.72$ \\
\hline & & & $73.0-76.0$ & $99.12-107.72$ \\
\hline & & 4 & $5.0-7.0$ & $99.12-107.72$ \\
\hline & & & $63.0-65.0$ & $99.12-107.72$ \\
\hline & & 6 & $12.0-14.0$ & $99.12-107.72$ \\
\hline & & & $119.5-122.0$ & $99.12-107.72$ \\
\hline & 4 & 1 & $51.0-54.5$ & $189.51-190.43$ \\
\hline & & & $140.0-143.5$ & $189.51-190.43$ \\
\hline & 5 & 1 & $69.0-71.0$ & $210.69-212.60$ \\
\hline & & 2 & 9.0-11.0 & $210.69-212.60$ \\
\hline & & & $111.0-113.0$ & $210.69-212.60$ \\
\hline $85 \mathrm{~A}$ & 2 & 1 & 0.0 & 282.0 \\
\hline 86 & 1 & 1 & $9.0-10.0$ & 13.09 \\
\hline & & & $18.0-20.0$ & $13.18-16.76$ \\
\hline & & & $75.0-76.0$ & $13.18-16.76$ \\
\hline & & & $99.0-101.0$ & $13.18-16.76$ \\
\hline & & 3 & $9.0-11.0$ & $13.18-16.76$ \\
\hline & & & $74.0-76.0$ & $13.18-16.76$ \\
\hline & 2 & 1 & $59.0-61.0$ & 52.60 \\
\hline & 3 & 1 & $20.0-22.0$ & $161.20-169.26$ \\
\hline & & & $75.0-77.0$ & $161.20-169.26$ \\
\hline & & 2 & $16.0-18.0$ & $161.20-169.26$ \\
\hline & & & $74.0-76.0$ & $161.20-169.26$ \\
\hline & & 3 & $8.0-10.0$ & $161.20-169.26$ \\
\hline & & & $74.0-76.0$ & $161.20-169.26$ \\
\hline & & 4 & $6.0-8.0$ & $161.20-169.26$ \\
\hline & & & $74.0-76.0$ & $161.20-169.26$ \\
\hline & & 5 & $5.0-7.0$ & $161.20-169.26$ \\
\hline & & & $75.0-77.0$ & $161.20-169.26$ \\
\hline & & 6 & $16.0-18.0$ & $161.20-169.26$ \\
\hline & & & $74.0-76.0$ & $161.20-169.26$ \\
\hline & 4 & 1 & $30.0-32.0$ & $257.30-263.75$ \\
\hline & & & $75.0-77.0$ & $257.30-163.75$ \\
\hline & & 2 & $9.0-11.0$ & $257.30-163.75$ \\
\hline & & & $79.0-81.0$ & $257.30-163.75$ \\
\hline & & 3 & 9.0-11.0 & $257.30-263.75$ \\
\hline & & & $90.0-92.0$ & $257.30-263.75$ \\
\hline & & 4 & $16.0-18.0$ & $257.30-263.75$ \\
\hline & & & $82.0-84.0$ & $257.30-263.75$ \\
\hline & & 5 & $20.0-22.5$ & $257.30-263.75$ \\
\hline & & & $95.0-97.5$ & $257.30-263.75$ \\
\hline & 5 & 1 & $116.5-119.0$ & $372.16-376.26$ \\
\hline & & 2 & $15.0-17.5$ & $372.16-376.26$ \\
\hline & & & $75.0-77.0$ & $372.16-376.26$ \\
\hline
\end{tabular}

TABLE 14 - Continued

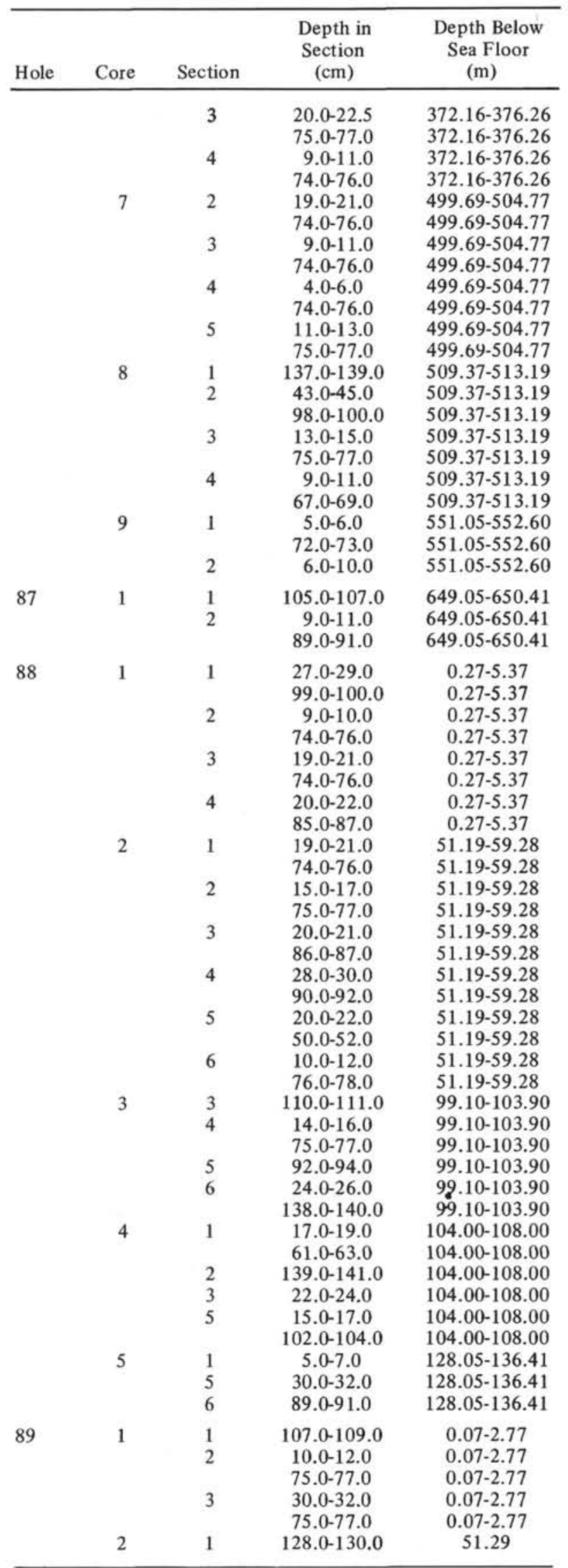


TABLE 14 - Continued

\begin{tabular}{|c|c|c|c|c|}
\hline Hole & Core & Section & $\begin{array}{l}\text { Depth in } \\
\text { Section } \\
\text { (cm) }\end{array}$ & $\begin{array}{l}\text { Depth Below } \\
\text { Sea Floor } \\
\text { (m) }\end{array}$ \\
\hline & 3 & 1 & $\begin{array}{c}7.0-9.0 \\
75.0770\end{array}$ & $\begin{array}{l}119.07-124.32 \\
119.07-124\end{array}$ \\
\hline & & 2 & $\begin{array}{l}75.0-77.0 \\
10.0-12.0\end{array}$ & $119.07-124.32$ \\
\hline & & & $75.0-77.0$ & $119.07-124.32$ \\
\hline & & 3 & $15.0-17.0$ & $119.07-124.32$ \\
\hline & & & $75.0-77.0$ & $119.07-124.32$ \\
\hline & & 4 & $15.0-17.0$ & $119.07-124.32$ \\
\hline & & & $80.0-82.0$ & $119.07-124.32$ \\
\hline & 4 & 1 & $22.0-24.0$ & $220.02-228.26$ \\
\hline & & & $77.0-79.0$ & $220.02-228.26$ \\
\hline & & 2 & $10.0-11.0$ & $220.02-228.26$ \\
\hline & & & $75.0-77.0$ & $220.02-228.26$ \\
\hline & & 3 & $10.0-12.0$ & $220.02-228.26$ \\
\hline & & & $75.0-77.0$ & $220.02-228.26$ \\
\hline & & 4 & $17.0-19.0$ & $220.02-228.26$ \\
\hline & & & $75.0-77.0$ & $220.02-228.26$ \\
\hline & & 5 & $39.0-41.0$ & $220.02-228.26$ \\
\hline & & & $126.0-128.0$ & $220.02-228.26$ \\
\hline & & 6 & $9.0-11.0$ & $220.02-228.26$ \\
\hline & & & $74.0-76.0$ & $220.02-228.26$ \\
\hline & 5 & 1 & $124.0-126.0$ & 300.25 \\
\hline & 6 & 3 & $24.0-26.0$ & $377.24-382.86$ \\
\hline & & & $75.0-77.0$ & $377.24-382.86$ \\
\hline & & 4 & $15.0-17.0$ & $377.24-382.86$ \\
\hline & & & $75.0-77.0$ & $377.24-382.86$ \\
\hline & & 5 & $15.0-17.0$ & $377.24-382.86$ \\
\hline & & & $84.0-86.0$ & $377.24-382.86$ \\
\hline \multirow[t]{39}{*}{90} & 1 & 1 & $15.0-17.0$ & $0.15-8.27$ \\
\hline & & & $90.0-92.0$ & $0.15-8.27$ \\
\hline & & 2 & $10.0-12.0$ & $0.15-8.27$ \\
\hline & & & $79.0-81.0$ & $0.15-8.27$ \\
\hline & & 3 & $9.0-10.0$ & $0.15-8.27$ \\
\hline & & & $75.0-77.0$ & $0.15-8.27$ \\
\hline & & 4 & $10.0-12.0$ & $0.15-8.27$ \\
\hline & & & $75.0-77.0$ & $0.15-8.27$ \\
\hline & & 5 & $10.0-12.0$ & $0.15-8.27$ \\
\hline & & & $75.0-77.0$ & $0.15-8.27$ \\
\hline & & 6 & $10.0-12.0$ & $0.15-8.27$ \\
\hline & & & $75.0-77.0$ & $0.15-8.27$ \\
\hline & 2 & 1 & $60.0-62.0$ & $70.60-74.72$ \\
\hline & & & $120.0-122.0$ & $70.60-74.72$ \\
\hline & & 2 & $20.0-22.0$ & $70.60-74.72$ \\
\hline & & & $75.0-76.0$ & $70.60-74.72$ \\
\hline & & 3 & $10.0-12.0$ & $70.60-74.72$ \\
\hline & & & $75.0-77.0$ & $70.60-74.72$ \\
\hline & & 4 & $10.0-12.0$ & $70.60-74.72$ \\
\hline & & & $20.0-22.0$ & $70.60-74.72$ \\
\hline & 3 & 1 & $75.0-77.0$ & $130.75-133.82$ \\
\hline & & & $110.0-112.0$ & $130.75-133.82$ \\
\hline & & 2 & $10.0-12.0$ & $130.75-133.82$ \\
\hline & & & $95.0-97.0$ & $130.75-133.82$ \\
\hline & & 3 & $10.0-12.0$ & $130.75-133.82$ \\
\hline & & & $80.0-82.0$ & $130.75-133.82$ \\
\hline & 4 & 1 & $141.0-143.0$ & $189.41-190.72$ \\
\hline & & 2 & $10.0-12.0$ & $189.41-190.72$ \\
\hline & & & $75.0-77.0$ & $189.41-190.72$ \\
\hline & 5 & 1 & $20.0-22.0$ & $236.20-241.27$ \\
\hline & & & $75.0-77.0$ & $236.20-241.27$ \\
\hline & & 2 & $10.0-12.0$ & $236.20-241.27$ \\
\hline & & & $75.0-77.0$ & $236.20-241.27$ \\
\hline & & 3 & $10.0-21.0$ & $236.20-241.27$ \\
\hline & & & $75.0-77.0$ & $236.20-241.27$ \\
\hline & & 4 & $21.0-23.0$ & $236.20-241.27$ \\
\hline & & & $75.0-77.0$ & $236.20-241.27$ \\
\hline & 6 & 1 & $30.0-32.0$ & $293.30-295.27$ \\
\hline & & & $76.0-78.0$ & $293.30-295.27$ \\
\hline
\end{tabular}

TABLE 14 - Continued

\begin{tabular}{|c|c|c|c|c|}
\hline Hole & Core & Section & $\begin{array}{l}\text { Depth in } \\
\text { Section } \\
\text { (cm) }\end{array}$ & $\begin{array}{l}\text { Depth Below } \\
\text { Sea Floor } \\
\text { (m) }\end{array}$ \\
\hline & & 2 & $\begin{array}{l}10.0-12.0 \\
75.0-77.0\end{array}$ & $\begin{array}{l}293.30-295.27 \\
293.30-295.27\end{array}$ \\
\hline & 7 & 1 & $49.0-52.0$ & $341.49-344.77$ \\
\hline & & & $100.0-102.0$ & $341.49-344.77$ \\
\hline & & 2 & $10.0-12.0$ & $341.49-344.77$ \\
\hline & & & $75.0-77.0$ & $341.49-344.77$ \\
\hline & & 3 & $10.0-12.0$ & $341.49-344.77$ \\
\hline & & & $75.0-77.0$ & $341.49-344.77$ \\
\hline & 9 & 1 & $35.0-37.0$ & $471.35-472.07$ \\
\hline & & & $105.0-107.0$ & $471.35-472.07$ \\
\hline & 10 & 1 & $19.0-23.0$ & $595.19-595.93$ \\
\hline & & & $90.0-93.0$ & $595.19-595.93$ \\
\hline & 11 & 6 & $21.0-23.0$ & $682.71-683.19$ \\
\hline & & & $67.0-69.0$ & $682.71-683.19$ \\
\hline & 13 & 1 & $10.0-12.0$ & $763.10-766.46$ \\
\hline & & & $70.0-72.0$ & $763.10-766.46$ \\
\hline & & 3 & $44.0-46.0$ & $763.10-766.46$ \\
\hline \multirow[t]{49}{*}{91} & 1 & 2 & $10.0-12.0$ & $61.60-68.27$ \\
\hline & & & $75.0-77.0$ & $61.60-68.27$ \\
\hline & & 3 & $10.0-12.0$ & $61.60-68.27$ \\
\hline & & & $75.0-77.0$ & $61.60-68.27$ \\
\hline & & 4 & $10.0-12.0$ & $61.60-68.27$ \\
\hline & & & $75.0-77.0$ & $61.60-68.27$ \\
\hline & & 5 & $10.0-12.0$ & $61.60-68.27$ \\
\hline & & & $75.0-76.0$ & $61.60-68.27$ \\
\hline & & 6 & $10.0-12.0$ & $61.60-68.27$ \\
\hline & & & $75.0-77.0$ & $61.60-68.27$ \\
\hline & 2 & 2 & $10.0-12.0$ & $124.60-129.79$ \\
\hline & & & $75.0-77.0$ & $124.60-129.79$ \\
\hline & & 3 & $10.0-12.0$ & $124.60-129.79$ \\
\hline & & & $75.0-76.0$ & $124.60-129.79$ \\
\hline & & 4 & $10.0-12.0$ & $124.60-129.79$ \\
\hline & & & $75.0-77.0$ & $124.60-129.79$ \\
\hline & & 5 & $12.0-14.0$ & $124.60-129.79$ \\
\hline & & & $77.0-79.0$ & $124.60-129.79$ \\
\hline & 3 & 1 & $10.0-12.0$ & $159.10-167.24$ \\
\hline & & & $75.0-77.0$ & $159.10-167.24$ \\
\hline & & 2 & $20.0-22.0$ & $159.10-167.24$ \\
\hline & & 6 & $10.0-12.0$ & $159.10-167.24$ \\
\hline & & & $73.0-74.0$ & $159.10-167.24$ \\
\hline & 4 & 1 & $10.0-12.0$ & $177.10-185.27$ \\
\hline & & & $75.0-77.0$ & $177.10-185.27$ \\
\hline & & 2 & $10.0-12.0$ & $177.10-185.27$ \\
\hline & & & $75.0-77.0$ & $177.10-185.27$ \\
\hline & & 3 & $10.0-12.0$ & $177.10-185.27$ \\
\hline & & & $75.0-77.0$ & $177.10-185.27$ \\
\hline & & 4 & $10.0-12.0$ & $177.10-185.27$ \\
\hline & & & $95.0-97.0$ & $177.10-185.27$ \\
\hline & & 5 & $10.0-12.0$ & $177.10-185.27$ \\
\hline & & & $75.0-77.0$ & $177.10-185.27$ \\
\hline & & 6 & $20.0-22.0$ & $177.10-185.27$ \\
\hline & & & $75.0-77.0$ & $177.10-185.27$ \\
\hline & 5 & 1 & $20.0-22.0$ & $186.20-194.27$ \\
\hline & & & $75.0-76.0$ & $186.20-194.27$ \\
\hline & & 2 & $20.0-22.0$ & $186.20-194.27$ \\
\hline & & & $75.0-77.0$ & $186.20-194.27$ \\
\hline & & 3 & $20.0-22.0$ & $186.20-194.27$ \\
\hline & & & $75.0-77.0$ & $186.20-194.27$ \\
\hline & & 4 & $20.0-22.0$ & $186.20-194.27$ \\
\hline & & & $75.0-77.0$ & $186.20-194.27$ \\
\hline & & 5 & $20.0-22.0$ & $186.20-194.27$ \\
\hline & & & $75.0-77.0$ & $186.20-194.27$ \\
\hline & & 6 & $20.0-22.0$ & $186.20-194.27$ \\
\hline & & & $75.0-77.0$ & $186.20-194.27$ \\
\hline & 6 & 1 & $20.0-22.0$ & $301.20-308.07$ \\
\hline & & & $75.0-77.0$ & $301.20-308.07$ \\
\hline
\end{tabular}


TABLE 14 - Continued

\begin{tabular}{|c|c|c|c|c|}
\hline Hole & Core & Section & $\begin{array}{l}\text { Depth in } \\
\text { Section } \\
(\mathrm{cm})\end{array}$ & $\begin{array}{l}\text { Depth Below } \\
\text { Sea Floor } \\
(\mathrm{m})\end{array}$ \\
\hline & & 2 & $20.0-22.0$ & $301.20-308.07$ \\
\hline & & & $95.0-97.0$ & $301.20-308.07$ \\
\hline & & 2 & $10.0-12.0$ & $891.68-893.62$ \\
\hline & & & $75.0-77.0$ & $891.68-893.62$ \\
\hline & & 3 & $20.0-22.0$ & $891.68-893.62$ \\
\hline \multirow[t]{40}{*}{92} & 2 & 1 & $100.0-102.0$ & $31.50-35.77$ \\
\hline & & 2 & $10.0-12.0$ & $31.50-35.77$ \\
\hline & & & $75.0-77.0$ & $31.50-35.77$ \\
\hline & & 3 & $10.0-12.0$ & $31.50-35.77$ \\
\hline & & 4 & $10.0-12.0$ & $31.50-35.77$ \\
\hline & & & $75.0-77.0$ & $31.50-35.77$ \\
\hline & & 5 & $13.0-15.0$ & $31.50-35.77$ \\
\hline & & & $75.0-77.0$ & $31.50-35.77$ \\
\hline & 3 & 1 & $120.0-122.0$ & $88.20-93.77$ \\
\hline & & 2 & $10.0-12.0$ & $88.20-93.77$ \\
\hline & & & $75.0-77.0$ & $88.20-93.77$ \\
\hline & & 3 & $10.0-12.0$ & $88.20-93.77$ \\
\hline & & & $75.0-77.0$ & $88.20-93.77$ \\
\hline & & 4 & $10.0-12.0$ & $88.20-93.77$ \\
\hline & & & $75.0-77.0$ & $88.20-93.77$ \\
\hline & & 5 & $10.0-12.0$ & $88.20-93.77$ \\
\hline & & & $75.0-77.0$ & $88.20-93.77$ \\
\hline & 4 & 1 & $10.0-12.0$ & $125.10-131.77$ \\
\hline & & & $75.0-77.0$ & $125.10-131.77$ \\
\hline & & 2 & $40.0-42.0$ & $125.10-131.77$ \\
\hline & & & $75.0-77.0$ & $125.10-131.77$ \\
\hline & & 3 & $10.0-12.0$ & $125.10-131.77$ \\
\hline & & & $75.0-77.0$ & $125.10-131.77$ \\
\hline & & 5 & $10.0-12.0$ & $125.10-131.77$ \\
\hline & & & $75.0-77.0$ & $125.10-131.77$ \\
\hline & 5 & 1 & $10.0-12.0$ & $173.10-181.27$ \\
\hline & & & $75.0-77.0$ & $173.10-181.27$ \\
\hline & & 2 & $10.0-12.0$ & $173.10-181.27$ \\
\hline & & & $75.0-77.0$ & $173.10-181.27$ \\
\hline & & 3 & $10.0-12.0$ & $173.10-181.27$ \\
\hline & & & $75.0-77.0$ & $173.10-181.27$ \\
\hline & & 4 & $7.0-9.0$ & $173.10-181.27$ \\
\hline & & & $75.0-77.0$ & $173.10-181.27$ \\
\hline & & 5 & $10.0-12.0$ & $173.10-181.27$ \\
\hline & & & $75.0-77.0$ & $173.10-181.27$ \\
\hline & & 6 & $10.0-12.0$ & $173.10-181.27$ \\
\hline & & & $75.0-77.0$ & $173.10-181.27$ \\
\hline & 6 & 2 & $71.0-73.0$ & $222.21-222.90$ \\
\hline & & & $138.0-140.0$ & $222.21-222.90$ \\
\hline & 8 & 1 & $83.0-85.0$ & 262.84 \\
\hline \multirow[t]{2}{*}{93} & 1 & 2 & $10.0-12.0$ & 0.11 \\
\hline & & & $75.0-77.0$ & 0.76 \\
\hline \multirow[t]{19}{*}{94} & 1 & 1 & $130.0-132.0$ & $1.30-2.27$ \\
\hline & & 2 & $10.0-12.0$ & $1.30-2.27$ \\
\hline & & & $75.0-77.0$ & $1.30-2.27$ \\
\hline & 2 & 1 & $10.0-12.0$ & $52.10-60.27$ \\
\hline & & & $75.0-77.0$ & $52.10-60.27$ \\
\hline & & 2 & $10.0-12.0$ & $52.10-60.27$ \\
\hline & & & $75.0-77.0$ & $52.10-60.27$ \\
\hline & & 3 & $10.0-12.0$ & $52.10-60.27$ \\
\hline & & & $75.0-77.0$ & $52.10-60.27$ \\
\hline & & 4 & $10.0-12.0$ & $52.10-60.27$ \\
\hline & & & $75.0-77.0$ & $52.10-60.27$ \\
\hline & & 5 & $10.0-12.0$ & $52.10-60.27$ \\
\hline & & & $75.0-77.0$ & $52.10-60.27$ \\
\hline & & 6 & $10.0-12.0$ & $52.10-60.27$ \\
\hline & & & $75.0-77.0$ & $52.10-60.27$ \\
\hline & 3 & 1 & $75.0-77.0$ & $100.75-108.27$ \\
\hline & & 2 & $10.0-12.0$ & $100.75-108.27$ \\
\hline & & & $75.0-77.0$ & $100.75-108.27$ \\
\hline & & 3 & $10.0-12.0$ & $100.75-108.27$ \\
\hline
\end{tabular}

TABLE 14 - Continued

\begin{tabular}{|c|c|c|c|c|}
\hline Hole & Core & Section & $\begin{array}{l}\text { Depth in } \\
\text { Section } \\
\text { (cm) }\end{array}$ & $\begin{array}{l}\text { Depth Below } \\
\text { Sea Floor } \\
\text { (m) }\end{array}$ \\
\hline & & & $75.0-77.0$ & $100.75-108.27$ \\
\hline \multirow{7}{*}{\multicolumn{2}{|c|}{25}} & 1 & $128.0-130.0$ & $891.68-893.62$ \\
\hline & & 3 & $10.0-12.0$ & $301.20-308.07$ \\
\hline & & & $75.0-77.0$ & $301.20-308.07$ \\
\hline & & 4 & $10.0-12.0$ & $301.20-308.07$ \\
\hline & & & $75.0-77.0$ & $301.20-308.07$ \\
\hline & & 5 & $25.0-27.0$ & $301.20-308.07$ \\
\hline & & & $105.0-107.0$ & $301.20-308.07$ \\
\hline \multirow{10}{*}{\multicolumn{2}{|c|}{7}} & 1 & $80.0-82.0$ & $407.80-413.77$ \\
\hline & & & $120.0-122.0$ & $407.80-413.77$ \\
\hline & & 2 & $40.0-42.0$ & $407.80-413.77$ \\
\hline & & & $120.0-122.0$ & $407.80-413.77$ \\
\hline & & 3 & $20.0-22.0$ & $407.80-413.77$ \\
\hline & & & $95.0-97.0$ & $407.80-413.77$ \\
\hline & & 4 & $10.0-12.0$ & $407.80-413.77$ \\
\hline & & & $75.0-77.0$ & $407.80-413.77$ \\
\hline & & 5 & $10.0-12.0$ & $407.80-413.77$ \\
\hline & & & $75.0-77.0$ & $407.80-413.77$ \\
\hline \multirow{4}{*}{\multicolumn{2}{|c|}{8}} & 1 & $6.0-8.0$ & $490.66-492.27$ \\
\hline & & & $75.0-77.0$ & $490.66-492.27$ \\
\hline & & 2 & $10.0-12.0$ & $490.66-492.27$ \\
\hline & & & $75.0-77.0$ & $490.66-492.27$ \\
\hline \multirow{9}{*}{\multicolumn{2}{|c|}{9}} & 1 & $15.0-16.0$ & $530.45-537.62$ \\
\hline & & & $75.0-77.0$ & $530.45-537.62$ \\
\hline & & 2 & $10.0-12.0$ & $530.45-537.62$ \\
\hline & & & $75.0-77.0$ & $530.45-537.62$ \\
\hline & & 3 & $10.0-12.0$ & $530.45-537.62$ \\
\hline & & & $75.0-77.0$ & $530.45-537.62$ \\
\hline & & 4 & $10.0-12.0$ & $530.45-537.62$ \\
\hline & & & $75.0-77.0$ & $530.45-537.62$ \\
\hline & & 6 & $10.0-12.0$ & $530.45-537.62$ \\
\hline \multirow{4}{*}{\multicolumn{2}{|c|}{10}} & 2 & $10.0-12.0$ & $653.60-657.19$ \\
\hline & & & $75.0-77.0$ & $653.60-657.19$ \\
\hline & & 4 & $10.0-12.0$ & $653.60-657.19$ \\
\hline & & & $68.0-69.0$ & $653.60-657.19$ \\
\hline \multirow{5}{*}{\multicolumn{2}{|c|}{11}} & 2 & $10.0-12.0$ & $771.60-775.27$ \\
\hline & & & $75.0-77.0$ & $771.60-775.27$ \\
\hline & & 3 & $72.0-74.0$ & $771.60-775.27$ \\
\hline & & 4 & $10.0-12.0$ & $771.60-775.27$ \\
\hline & & & $75.0-77.0$ & $771.60-775.27$ \\
\hline \multirow{2}{*}{\multicolumn{2}{|c|}{12}} & 3 & $30.0-32.0$ & $782.50-783.00$ \\
\hline & & & $78.0-80.0$ & $782.50-783.00$ \\
\hline \multirow{4}{*}{\multicolumn{2}{|c|}{13}} & 1 & $68.0-70.0$ & $789.08-792.17$ \\
\hline & & & $104.0-106.0$ & $789.08-792.17$ \\
\hline & & 2 & $75.0-77.0$ & $789.08-792.17$ \\
\hline & & 3 & $75.0-77.0$ & $789.08-792.17$ \\
\hline \multirow{2}{*}{\multicolumn{2}{|c|}{14}} & 2 & $20.0-22.0$ & $799.30-800.32$ \\
\hline & & & $120.0-122.0$ & $799.30-800.32$ \\
\hline \multirow{6}{*}{\multicolumn{2}{|c|}{15}} & 1 & $10.0-12.0$ & $806.90-810.57$ \\
\hline & & & $75.0-77.0$ & $806.90-810.57$ \\
\hline & & 2 & $10.0-12.0$ & $806.90-810.57$ \\
\hline & & & $75.0-77.0$ & $806.90-810.57$ \\
\hline & & 3 & $10.0-12.0$ & $806.90-810.57$ \\
\hline & & & $75.0-77.0$ & $806.90-810.57$ \\
\hline \multirow{5}{*}{\multicolumn{2}{|c|}{16}} & 1 & $24.0-26.0$ & $816.24-819.77$ \\
\hline & & 2 & $10.0-12.0$ & $816.24-819.77$ \\
\hline & & & $75.0-77.0$ & $816.24-819.77$ \\
\hline & & 3 & $10.0-12.0$ & $816.24-819.77$ \\
\hline & & & $75.0-77.0$ & $816.24-819.77$ \\
\hline & 18 & 6 & $82.0-84.0$ & 842.73 \\
\hline \multirow{4}{*}{\multicolumn{2}{|c|}{20}} & 2 & $10.0-12.0$ & $854.40-861.07$ \\
\hline & & & $75.0-77.0$ & $854.40-861.07$ \\
\hline & & 6 & $10.0-12.0$ & $854.40-861.07$ \\
\hline & & & $75.0-77.0$ & $854.40-861.07$ \\
\hline & 22 & 1 & $10.0-12.0$ & $866.10-868.28$ \\
\hline & & & $75.0-77.0$ & $866.10-868.28$ \\
\hline & & 2 & $22.0-24.0$ & $866.10-868.28$ \\
\hline & & & $76.0-78.0$ & $866.10-868.28$ \\
\hline
\end{tabular}


TABLE 14 - Continued

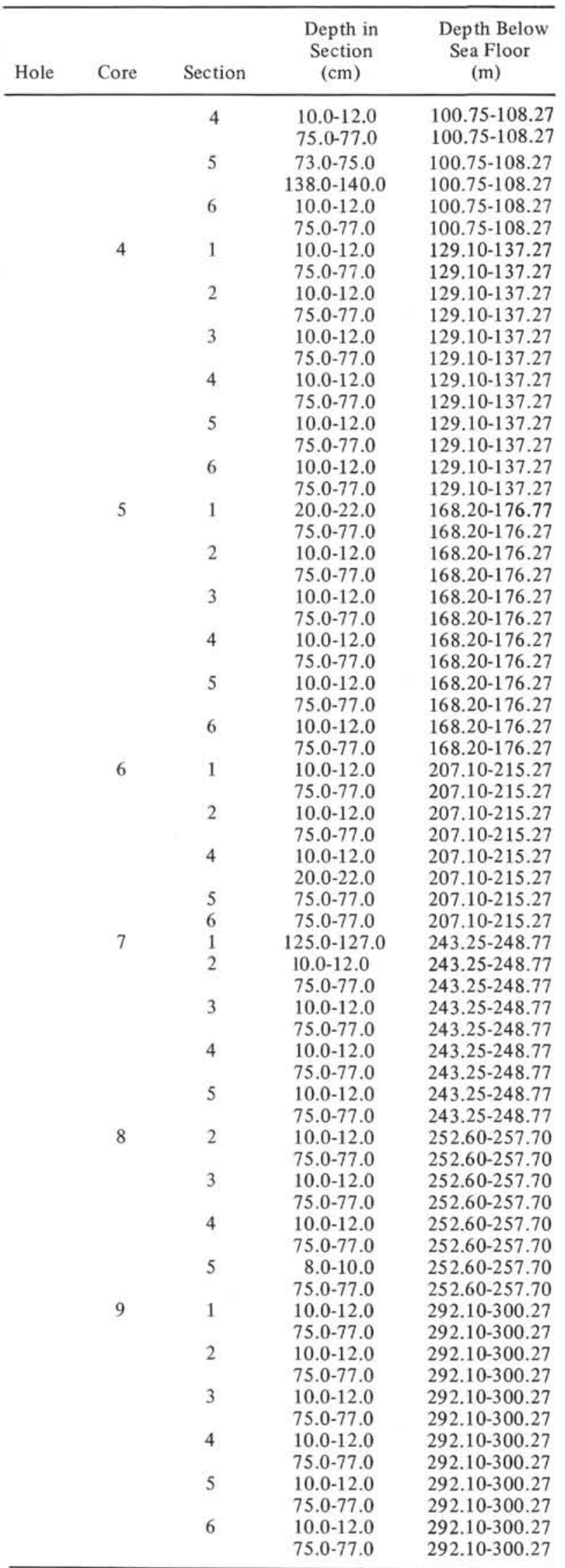

TABLE 14 - Continued

\begin{tabular}{|c|c|c|c|c|}
\hline Hole & Core & Section & $\begin{array}{l}\text { Depth in } \\
\text { Section } \\
(\mathrm{cm})\end{array}$ & $\begin{array}{l}\text { Depth Below } \\
\text { Sea Floor } \\
\text { (m) }\end{array}$ \\
\hline & \multirow[t]{5}{*}{10} & 1 & $110.0-112.0$ & $332.10-334.77$ \\
\hline & & 2 & $10.0-12.0$ & $332.10-334.77$ \\
\hline & & & $75.0-77.0$ & $332.10-334.77$ \\
\hline & & 3 & $10.0-12.0$ & $332.10-334.77$ \\
\hline & & & $75.0-77.0$ & $332.10-334.77$ \\
\hline & \multirow[t]{10}{*}{11} & 2 & $10.0-12.0$ & $364.60-369.00$ \\
\hline & & & $75.0-77.0$ & $364.60-369.00$ \\
\hline & & 3 & $10.0-12.0$ & $364.60-369.00$ \\
\hline & & & $75.0-77.0$ & $364.60-369.00$ \\
\hline & & 4 & $10.0-12.0$ & $364.60-369.00$ \\
\hline & & & $75.0-77.0$ & $364.60-369.00$ \\
\hline & & 5 & $10.0-12.0$ & $364.60-369.00$ \\
\hline & & & $75.0-77.0$ & $364.60-369.00$ \\
\hline & & 6 & $10.0-12.0$ & $364.60-369.00$ \\
\hline & & & $75.0-77.0$ & $364.60-369.00$ \\
\hline & \multirow[t]{2}{*}{12} & 4 & $10.0-12.0$ & $373.60-374.27$ \\
\hline & & & $75.0-77.0$ & $373.60-374.27$ \\
\hline & \multirow[t]{2}{*}{13} & 3 & $10.0-12.0$ & $381.10-381.77$ \\
\hline & & & $75.0-77.0$ & $381.10-381.77$ \\
\hline & \multirow[t]{2}{*}{14} & 4 & $10.0-12.0$ & $410.60-411.27$ \\
\hline & & & $75.0-77.0$ & $410.60-411.27$ \\
\hline & \multirow[t]{2}{*}{15} & 4 & $54.0-56.0$ & $417.04-417.83$ \\
\hline & & & $131.0-133.0$ & $417.04-417.83$ \\
\hline & \multirow[t]{2}{*}{16} & 3 & $10.0-12.0$ & $421.10-421.77$ \\
\hline & & & $75.0-77.0$ & $421.10-421.77$ \\
\hline & \multirow[t]{2}{*}{17} & 5 & $10.0-12.0$ & $430.10-430.77$ \\
\hline & & & $75.0-77.0$ & $430.10-430.77$ \\
\hline & \multirow[t]{2}{*}{18} & 4 & $10.0-12.0$ & $437.60-438.27$ \\
\hline & & & $75.0-77.0$ & $437.60-438.27$ \\
\hline & \multirow[t]{2}{*}{19} & 5 & $10.0-12.0$ & $448.10-448.77$ \\
\hline & & & $75.0-77.0$ & $448.10-448.77$ \\
\hline & \multirow[t]{2}{*}{20} & 2 & $10.0-12.0$ & $452.60-453.27$ \\
\hline & & & $75.0-77.0$ & $452.60-453.27$ \\
\hline & \multirow[t]{2}{*}{21} & 2 & $10.0-12.0$ & $461.60-462.27$ \\
\hline & & & $75.0-77.0$ & $461.60-462.27$ \\
\hline & \multirow[t]{2}{*}{22} & 4 & $10.0-12.0$ & $473.60-474.27$ \\
\hline & & & $75.0-77.0$ & $473.60-474.27$ \\
\hline & 23 & 2 & $10.0-12.0$ & $479.60-480.27$ \\
\hline & & & $75.0-77.0$ & $479.60-480.27$ \\
\hline & 24 & 3 & $10.0-12.0$ & $490.10-490.77$ \\
\hline & & & $75.0-77.0$ & $490.10-490.77$ \\
\hline & 25 & 3 & $10.0-12.0$ & $499.10-499.77$ \\
\hline & & & $75.0-77.0$ & $499.10-499.77$ \\
\hline & 26 & 3 & $10.0-12.0$ & $503.10-503.77$ \\
\hline & & & $75.0-77.0$ & $503.10-503.77$ \\
\hline & 28 & 5 & $10.0-12.0$ & $538.10-538.77$ \\
\hline & & & $75.0-77.0$ & $538.10-538.77$ \\
\hline & 30 & 1 & $70.0-72.0$ & 571.71 \\
\hline & 33 & 1 & $130.0-132.0$ & $613.30-613.72$ \\
\hline & & 2 & $40.0-42.0$ & $613.60-613.72$ \\
\hline & 34 & 1 & $72.0-74.0$ & 616.73 \\
\hline & 35 & 1 & $128.0-130.0$ & 626.29 \\
\hline & 36 & 1 & 0.0 & 627.00 \\
\hline & 39 & 1 & $95.0-97.0$ & 643.96 \\
\hline 95 & 1 & 1 & $75.0-77.0$ & $0.75-6.12$ \\
\hline & & 5 & $10.0-12.0$ & $0.75-6.12$ \\
\hline & 2 & 1 & $10.0-12.0$ & $82.10-90.27$ \\
\hline & & & $75.0-77.0$ & $82.10-90.27$ \\
\hline & & 2 & $20.0-22.0$ & $82.10-90.27$ \\
\hline & & & $75.0-77.0$ & $82.10-90.27$ \\
\hline & & 3 & $10.0-12.0$ & $82.10-90.27$ \\
\hline & & & $75.0-77.0$ & $82.10-90.27$ \\
\hline & & 6 & $10.0-12.0$ & $82.10-90.27$ \\
\hline & & & $75.0-77.0$ & $82.10-90.27$ \\
\hline & 3 & 1 & $10.0-12.0$ & $121.10-129.27$ \\
\hline & & & $75.0-77.0$ & $121.10-129.27$ \\
\hline & & 2 & $50.0-52.0$ & $121.10-129.27$ \\
\hline
\end{tabular}


TABLE 14 - Continued

\begin{tabular}{|c|c|c|c|c|}
\hline Hole & Core & Section & $\begin{array}{l}\text { Depth in } \\
\text { Section } \\
(\mathrm{cm})\end{array}$ & $\begin{array}{l}\text { Depth Below } \\
\text { Sea Floor } \\
\text { (m) }\end{array}$ \\
\hline & & 3 & $40.0-42.0$ & $121.10-129.27$ \\
\hline & & \multirow[t]{2}{*}{4} & $10.0-12.0$ & $121.10-129.27$ \\
\hline & & & $75.0-77.0$ & $121.10-129.27$ \\
\hline & & \multirow[t]{2}{*}{5} & $10.0-12.0$ & $121.10-129.27$ \\
\hline & & & $75.0-77.0$ & $121.10-129.27$ \\
\hline & & \multirow[t]{2}{*}{6} & $10.0-12.0$ & $121.10-129.27$ \\
\hline & & & $75.0-77.0$ & $121.10-129.27$ \\
\hline \multirow{6}{*}{\multicolumn{2}{|c|}{4}} & \multirow[t]{2}{*}{2} & $10.0-12.0$ & $160.60-164.27$ \\
\hline & & & $75.0-77.0$ & $160.60-164.27$ \\
\hline & & \multirow[t]{2}{*}{3} & $10.0-12.0$ & $160.60-164.27$ \\
\hline & & & $75.0-77.0$ & $160.60-164.27$ \\
\hline & & \multirow[t]{2}{*}{4} & $10.0-12.0$ & $160.60-164.27$ \\
\hline & & & $75.0-77.0$ & $160.60-164.27$ \\
\hline \multirow{8}{*}{\multicolumn{2}{|c|}{5}} & \multirow[t]{2}{*}{1} & $5.0-7.0$ & $198.05-203.27$ \\
\hline & & & $80.0-82.0$ & $198.05-203.27$ \\
\hline & & \multirow[t]{2}{*}{2} & $10.0-12.0$ & $198.05-203.27$ \\
\hline & & & $75.0-77.0$ & $198.05-203.27$ \\
\hline & & \multirow[t]{2}{*}{3} & $10.0-12.0$ & $198.05-203.27$ \\
\hline & & & $75.0-77.0$ & $198.05-203.27$ \\
\hline & & \multirow[t]{2}{*}{4} & $10.0-12.0$ & $198.05-203.27$ \\
\hline & & & $75.0-77.0$ & $198.05-203.27$ \\
\hline & 6 & 1 & $10.0-12.0$ & $236.10-244.27$ \\
\hline & & & $75.0-77.0$ & $236.10-244.27$ \\
\hline & & 2 & $10.0-12.0$ & $236.10-244.27$ \\
\hline & & & $75.0-77.0$ & $236.10-244.27$ \\
\hline & & 3 & $10.0-12.0$ & $236.10-244.27$ \\
\hline & & & $75.0-77.0$ & $236.10-244.27$ \\
\hline & & 4 & $10.0-12.0$ & $236.10-244.27$ \\
\hline & & & $75.0-77.0$ & $236.10-244.27$ \\
\hline & & 5 & $10.0-12.0$ & $236.10-244.27$ \\
\hline & & & $75.0-77.0$ & $236.10-244.27$ \\
\hline & & 6 & $10.0-12.0$ & $236.10-244.27$ \\
\hline & & & $75.0-77.0$ & $236.10-244.27$ \\
\hline & 7 & 1 & $10.0-12.0$ & $274.10-282.27$ \\
\hline & & & $75.0-77.0$ & $274.10-282.27$ \\
\hline & & 2 & $10.0-12.0$ & $274.10-282.27$ \\
\hline & & & $75.0-77.0$ & $274.10-282.27$ \\
\hline & & 3 & $10.0-12.0$ & $274.10-282.27$ \\
\hline & & & $75.0-77.0$ & $274.10-282.27$ \\
\hline & & 4 & $10.0-12.0$ & $274.10-282.27$ \\
\hline & & & $75.0-77.0$ & $274.10-282.27$ \\
\hline & & 5 & $10.0-12.0$ & $274.10-282.27$ \\
\hline & & & $75.0-77.0$ & $274.10-282.27$ \\
\hline & & 6 & $10.0-12.0$ & $274.10-282.27$ \\
\hline & & & $75.0-77.0$ & $274.10-282.27$ \\
\hline & 8 & 1 & $10.0-12.0$ & $332.10-340.27$ \\
\hline & & & $75.0-77.0$ & $332.10-340.27$ \\
\hline & & 2 & $10.0-12.0$ & $332.10-340.27$ \\
\hline & & & $75.0-77.0$ & $332.10-340.27$ \\
\hline & & 3 & $10.0-12.0$ & $332.10-340.27$ \\
\hline & & & $75.0-77.0$ & $332.10-340.27$ \\
\hline & & 4 & $10.0-12.0$ & $332.10-340.27$ \\
\hline & & & $75.0-77.0$ & $332.10-340.27$ \\
\hline & & 5 & $10.0-12.0$ & $332.10-340.27$ \\
\hline & & & $75.0-77.0$ & $332.10-340.27$ \\
\hline & & 6 & $15.0-17.0$ & $332.10-340.27$ \\
\hline & & & $75.0-77.0$ & $332.10-340.27$ \\
\hline & 10 & 1 & $15.0-17.0$ & $363.10-363.77$ \\
\hline & & & $75.0-77.0$ & $363.10-363.77$ \\
\hline & 11 & 1 & $118.0-120.0$ & $378.18-378.60$ \\
\hline & & 2 & $8.0-10.0$ & $378.18-378.60$ \\
\hline & 12 & 4 & $10.0-12.0$ & $390.60-391.27$ \\
\hline & & & $75.0-77.0$ & $390.60-391.27$ \\
\hline & 13 & 1 & $70.0-72.0$ & $395.70-396.15$ \\
\hline & & & $113.0-115.0$ & $395.70-396.15$ \\
\hline & 14 & 1 & $56.0-58.0$ & 400.57 \\
\hline & 15 & 6 & $10.0-12.0$ & $415.60-416.27$ \\
\hline & & & $75.0-77.0$ & $415.60-416.27$ \\
\hline
\end{tabular}

TABLE 14 - Continued

\begin{tabular}{|c|c|c|c|c|}
\hline Hole & Core & Section & $\begin{array}{l}\text { Depth in } \\
\text { Section } \\
(\mathrm{cm})\end{array}$ & $\begin{array}{l}\text { Depth Below } \\
\text { Sea Floor } \\
\text { (m) }\end{array}$ \\
\hline & 16 & 6 & $10.0-12.0$ & $424.60-425.27$ \\
\hline & & & $75.0-77.0$ & \\
\hline & 17 & 6 & $10.0-12.0$ & $433.60-434.27$ \\
\hline & & & $75.0-77.0$ & $433.60-434.27$ \\
\hline \multirow[t]{30}{*}{96} & 1 & 2 & $10.0-12.0$ & $102.60-109.27$ \\
\hline & & & $75.0-77.0$ & $102.60-109.27$ \\
\hline & & 3 & $10.0-12.0$ & $102.60-109.27$ \\
\hline & & & $75.0-77.0$ & $102.60-109.27$ \\
\hline & & 4 & $10.0-12.0$ & $102.60-109.27$ \\
\hline & & & $75.0-77.0$ & $102.60-109.27$ \\
\hline & & 5 & $10.0-12.0$ & $102.60-109.27$ \\
\hline & & & $75.0-77.0$ & $102.60-109.27$ \\
\hline & & 6 & $10.0-12.0$ & $102.60-109.27$ \\
\hline & & & $75.0-77.0$ & $102.60-109.27$ \\
\hline & 2 & 1 & $10.0-12.0$ & $199.10-207.27$ \\
\hline & & & $75.0-77.0$ & $199.10-207.27$ \\
\hline & & 5 & $10.0-12.0$ & $199.10-207.27$ \\
\hline & & & $75.0-77.0$ & $199.10-207.27$ \\
\hline & & 6 & $10.0-12.0$ & $199.10-207.27$ \\
\hline & & & $75.0-77.0$ & $199.10-207.27$ \\
\hline & 3 & 1 & $15.0-17.0$ & $301.15-309.27$ \\
\hline & & & $75.0-77.0$ & $301.15-309.27$ \\
\hline & & 2 & $10.0-12.0$ & $301.15-309.27$ \\
\hline & & & $75.0-77.0$ & $301.15-309.27$ \\
\hline & & 3 & $10.0-12.0$ & $301.15-309.27$ \\
\hline & & & $75.0-77.0$ & $301.15-309.27$ \\
\hline & & 4 & $10.0-12.0$ & $301.15-309.27$ \\
\hline & & & $75.0-77.0$ & $301.15-309.27$ \\
\hline & & 5 & $10.0-12.0$ & $301.15-309.27$ \\
\hline & & & $75.0-77.0$ & $301.15-309.27$ \\
\hline & & 6 & $15.0-17.0$ & $301.15-309.27$ \\
\hline & & & $75.0-77.0$ & $301.15-309.27$ \\
\hline & 5 & 1 & $20.0-22.0$ & $329.20-329.83$ \\
\hline & & & $81.0-83.0$ & $329.20-329.83$ \\
\hline \multirow[t]{34}{*}{97} & 1 & 2 & $10.0-12.0$ & $0.67-1.27$ \\
\hline & & & $75.0-77.0$ & $0.67-1.27$ \\
\hline & & 3 & $10.0-12.0$ & $2.10-4.27$ \\
\hline & & & $75.0-77.0$ & $2.10-4.27$ \\
\hline & & 4 & $10.0-12.0$ & $2.10-4.27$ \\
\hline & & & $75.0-77.0$ & $2.10-4.27$ \\
\hline & 2 & 1 & $20.0-22.0$ & $105.20-110.27$ \\
\hline & & & $75.0-77.0$ & $105.20-110.27$ \\
\hline & & 2 & $10.0-12.0$ & $105.20-110.27$ \\
\hline & & & $75.0-77.0$ & $105.20-110.27$ \\
\hline & & 3 & $10.0-12.0$ & $105.20-110.27$ \\
\hline & & & $75.0-77.0$ & $105.20-110.27$ \\
\hline & & 4 & $10.0-12.0$ & $105.20-110.27$ \\
\hline & & & $75.0-77.0$ & $105.20-110.27$ \\
\hline & 3 & 2 & $10.0-12.0$ & $143.60-145.77$ \\
\hline & & & $75.0-77.0$ & $143.60-145.77$ \\
\hline & & 3 & $10.0-12.0$ & $143.60-145.77$ \\
\hline & & & $75.0-77.0$ & $143.60-145.77$ \\
\hline & 4 & 2 & $100.0-102.0$ & $202.50-206.77$ \\
\hline & & 3 & $10.0-12.0$ & $202.50-206.77$ \\
\hline & & & $75.0-77.0$ & $202.50-206.77$ \\
\hline & & 4 & $10.0-12.0$ & $202.50-206.77$ \\
\hline & & & $75.0-77.0$ & $202.50-206.77$ \\
\hline & & 5 & $10.0-12.0$ & $202.50-206.77$ \\
\hline & & & $75.0-77.0$ & $202.50-206.77$ \\
\hline & 5 & 1 & $124.0-126.0$ & 251.25 \\
\hline & 6 & 1 & $85.0-87.0$ & 294.86 \\
\hline & 7 & 1 & $110.0-112.0$ & $306.10-306.77$ \\
\hline & & 2 & $25.0-27.0$ & $306.10-306.77$ \\
\hline & 8 & 1 & $38.0-40.0$ & $308.38-311.90$ \\
\hline & & & $54.0-56.0$ & $308.38-311.90$ \\
\hline & & 3 & $88.0-90.0$ & $308.83-311.90$ \\
\hline & 11 & 1 & 30.0 & 330.30 \\
\hline & 12 & 1 & $63.0-65.0$ & 333.64 \\
\hline
\end{tabular}


TABLE 15

Parameters for Semi-quantitative Determination of Mineral Concentrations Contained in the X-Ray Diffraction Data Reduction Program (MINILOG)

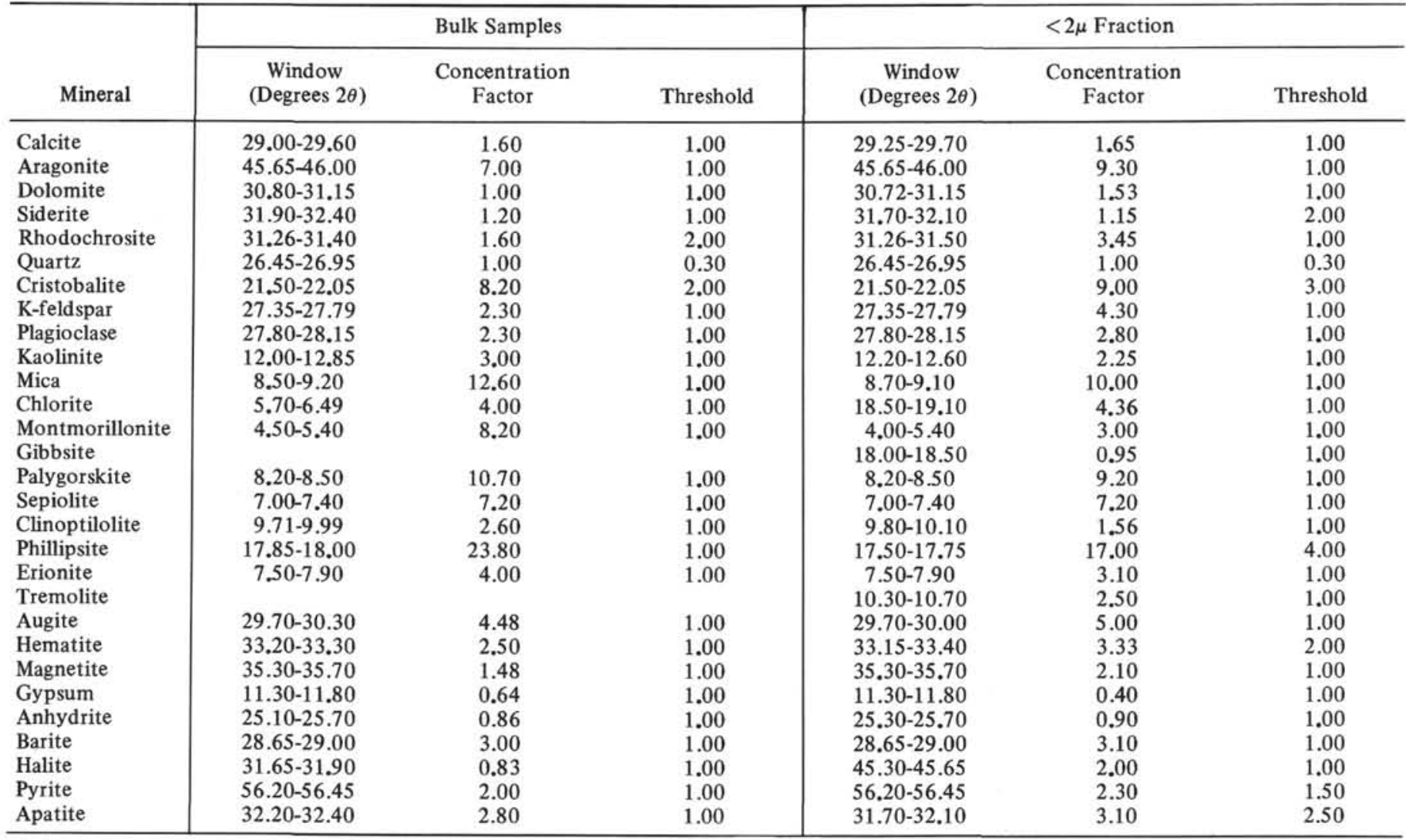

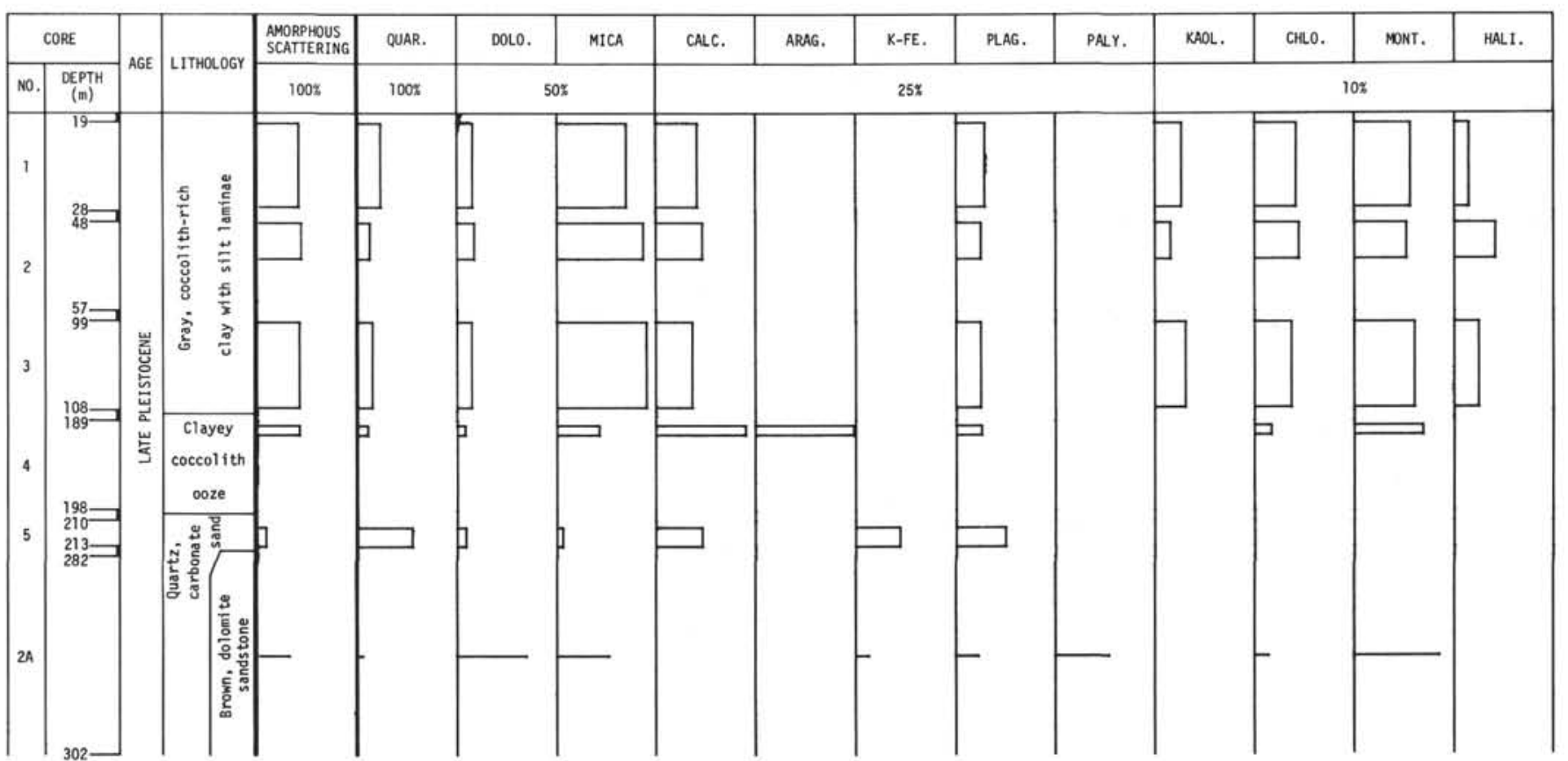

Figure 1. Site 85 bulk samples. 


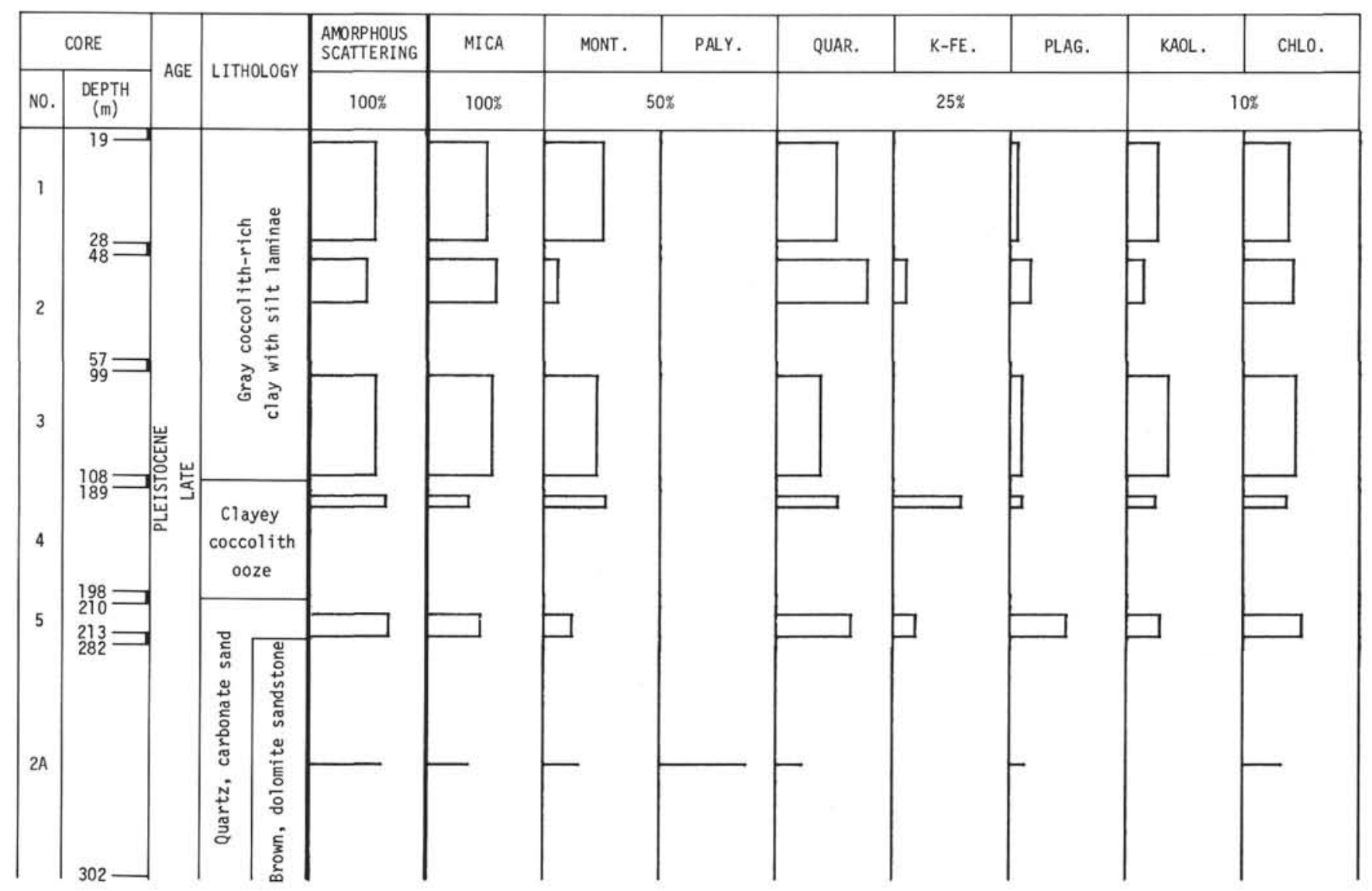

Figure 2. Site $85<2 \mu \mathrm{m}$ fractions.

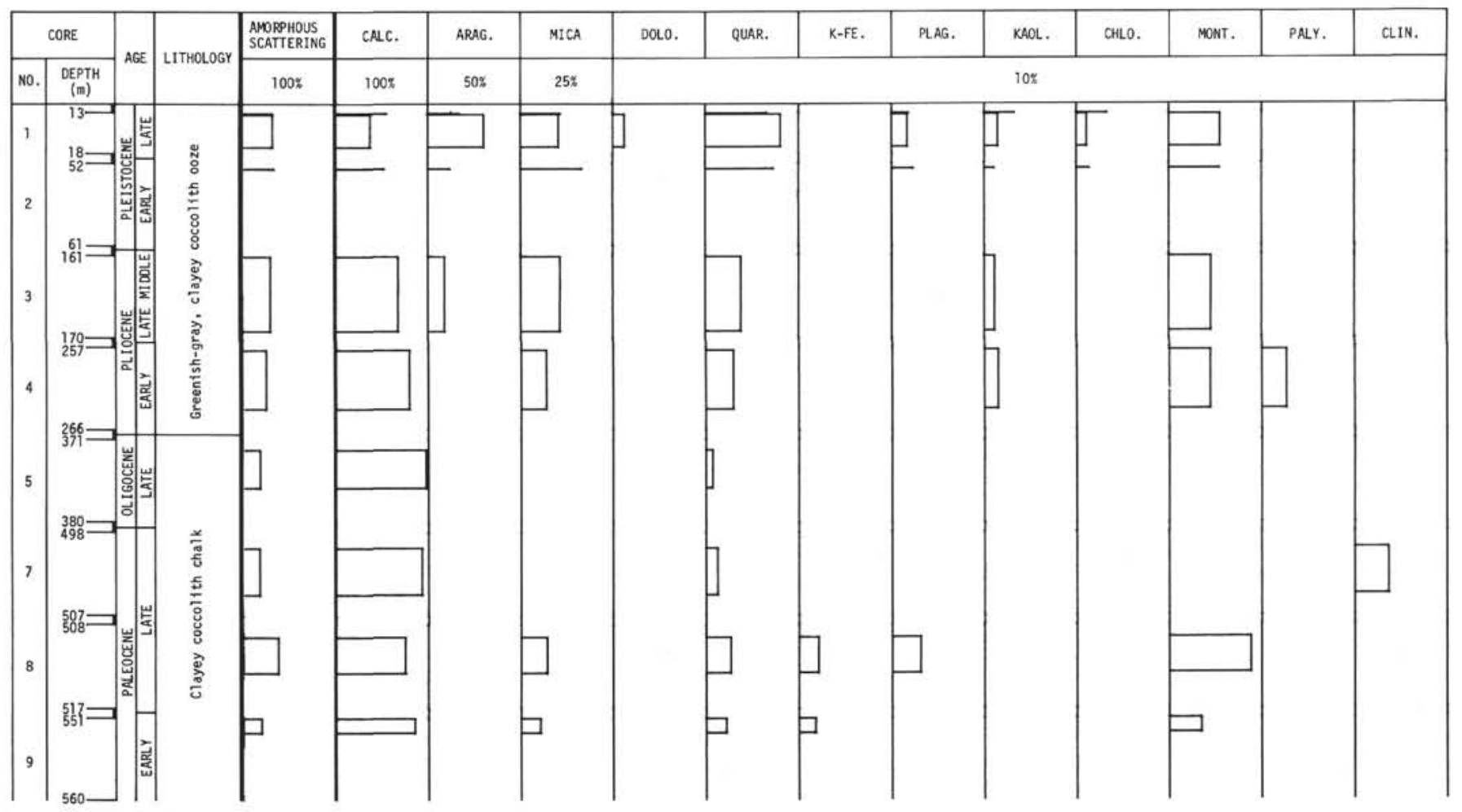

Figure 3. Site 86 bulk samples. 


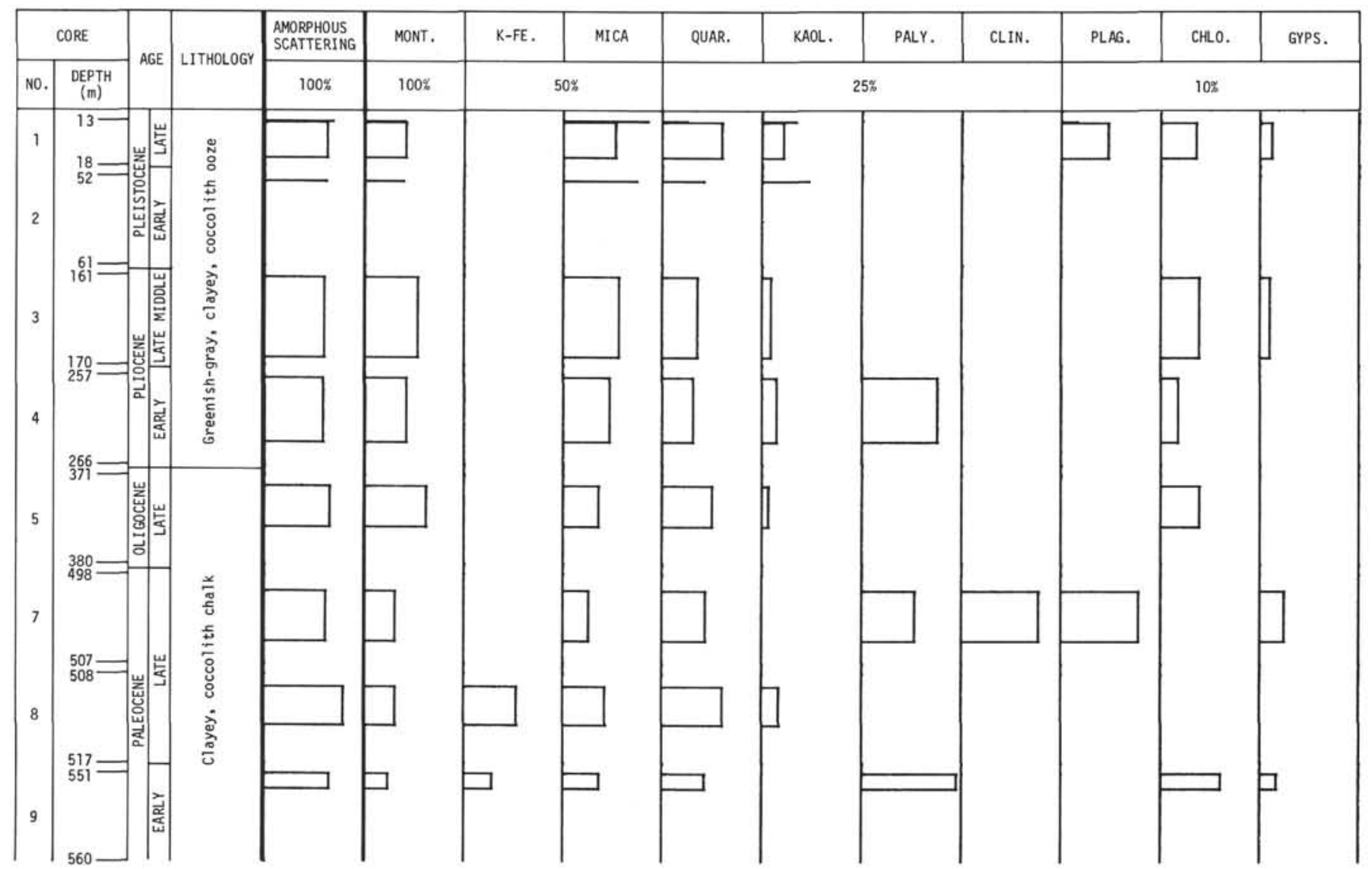

Figure 4. Site $86<2 \mu \mathrm{m}$ fractions.

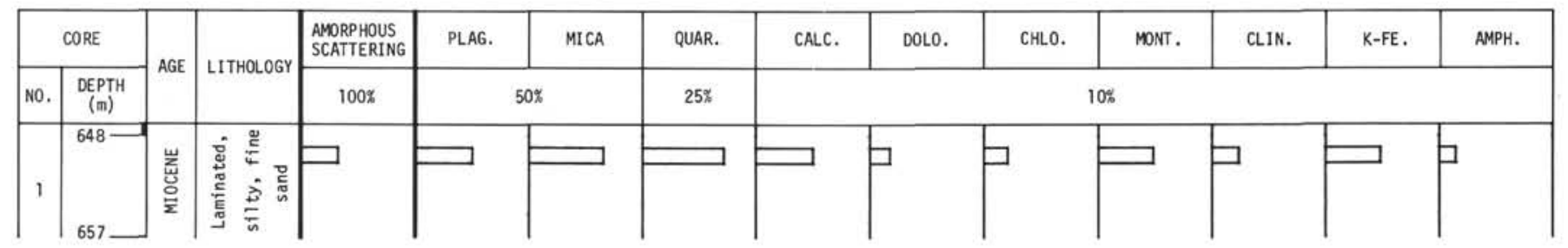

Figure 5. Site 87 bulk samples.

\begin{tabular}{|c|c|c|c|c|c|c|c|c|c|c|c|c|}
\hline \multicolumn{2}{|c|}{ CORE } & \multirow{2}{*}{ AGE } & \multirow{2}{*}{ LITHOLOGY } & $\begin{array}{l}\text { AMORPHOUS } \\
\text { SCATTERING }\end{array}$ & MICA & MONT. & PALY. & QUAR. & PLAG. & CHLO. & CLIN. & PYRI. \\
\hline No. & $\begin{array}{c}\text { DEPTH } \\
(\mathrm{m})\end{array}$ & & & $100 \%$ & \multicolumn{2}{|c|}{$50 \%$} & $25 \%$ & \multicolumn{5}{|c|}{$10 \%$} \\
\hline 1 & $\begin{array}{l}648- \\
657\end{array}$ & 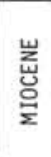 & 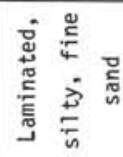 & $\square$ & $\square$ & & $\square$ & & & & ב & 口 \\
\hline
\end{tabular}

Figure 6. Site $87<2 \mu m$ fractions. 


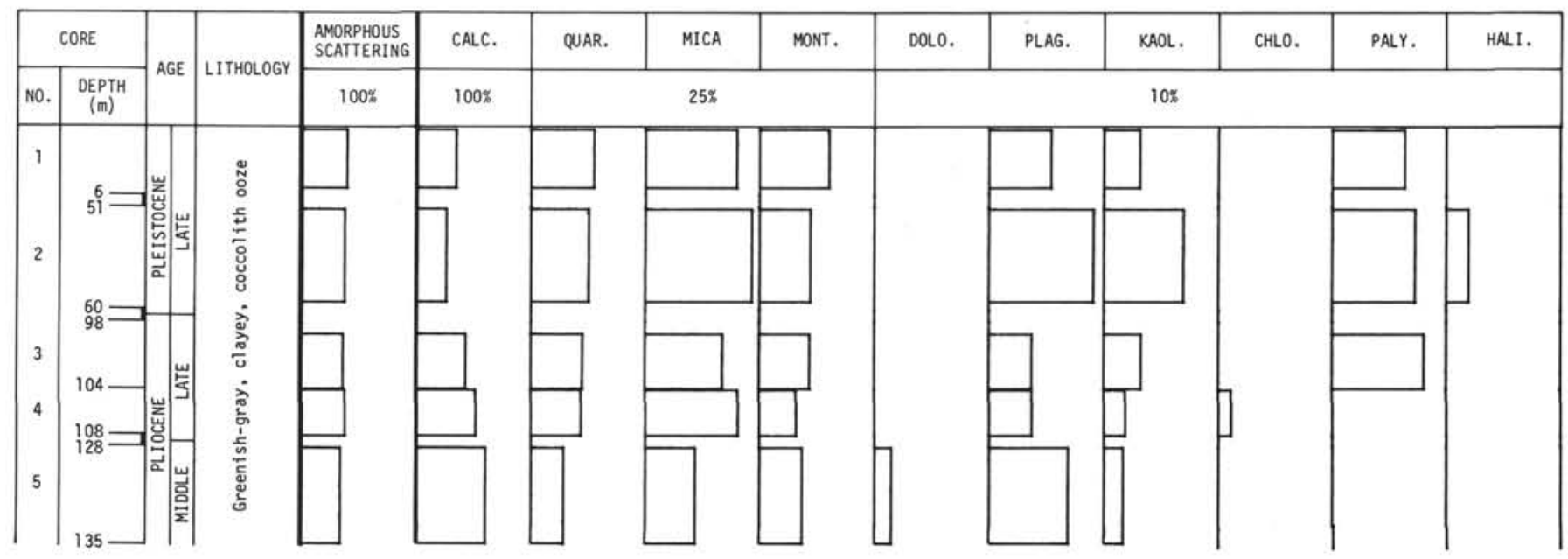

Figure 7. Site 88 bulk samples.

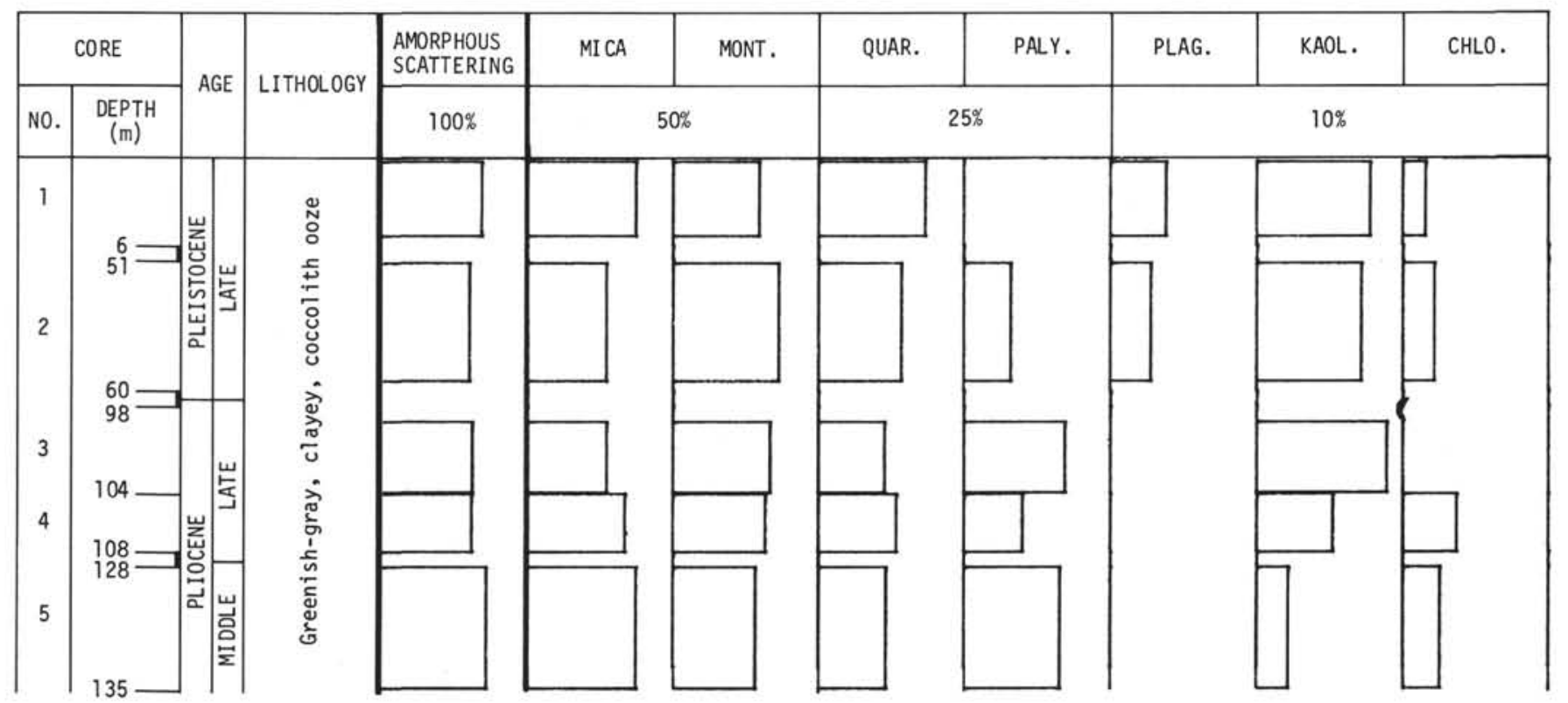

Figure 8. Site $88<2 \mu \mathrm{m}$ fractions.

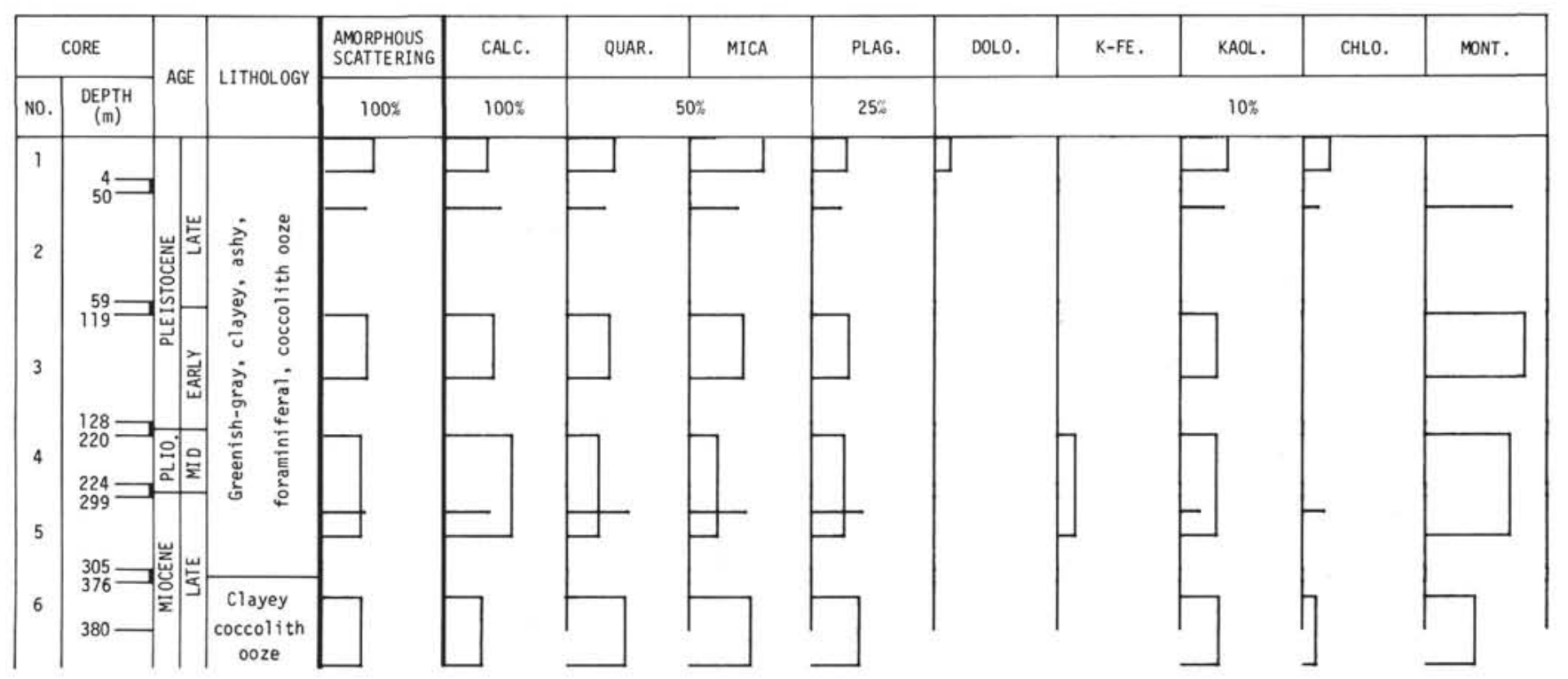

Figure 9. Site 89 bulk samples. 


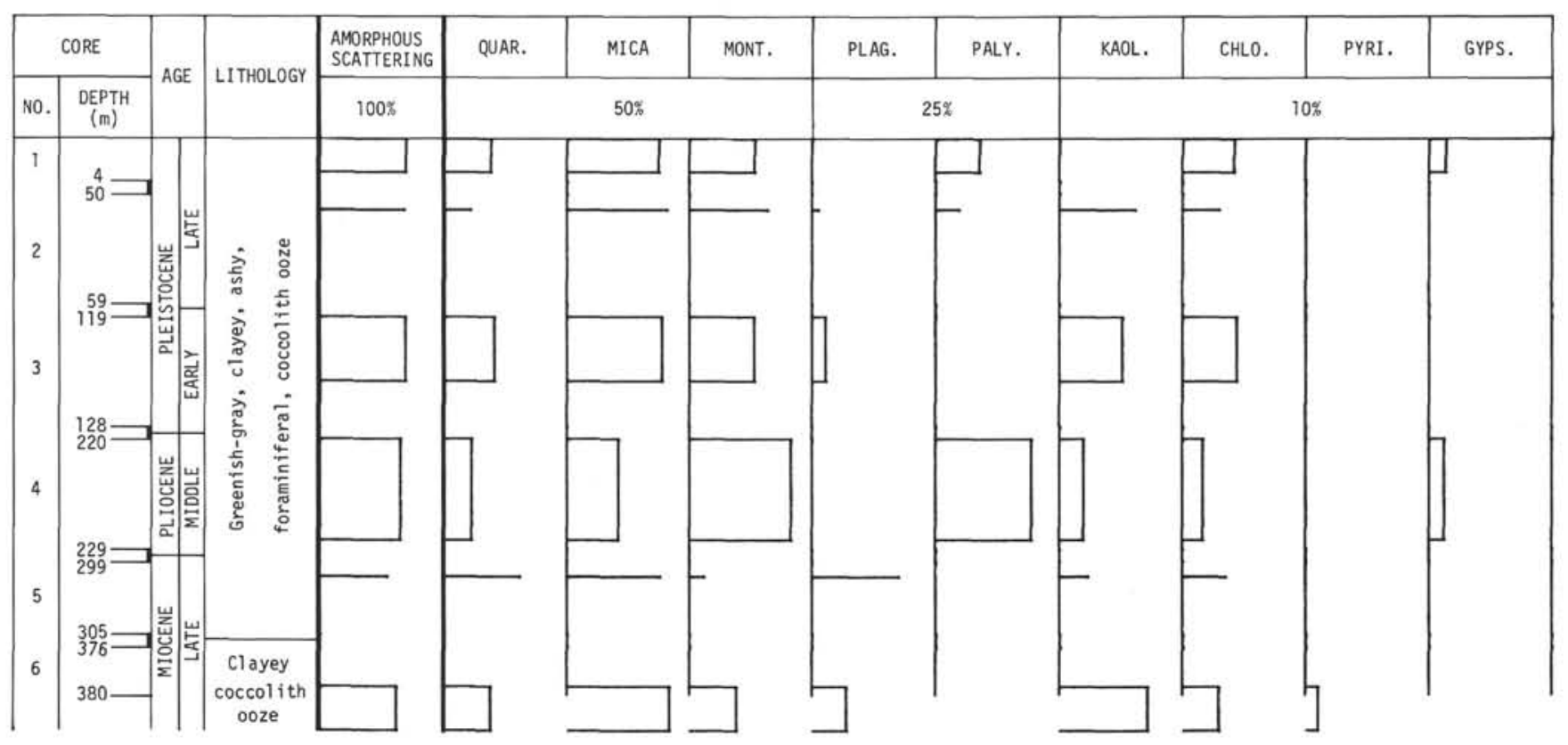

Figure 10. Site $89<2 \mu m$ fractions.

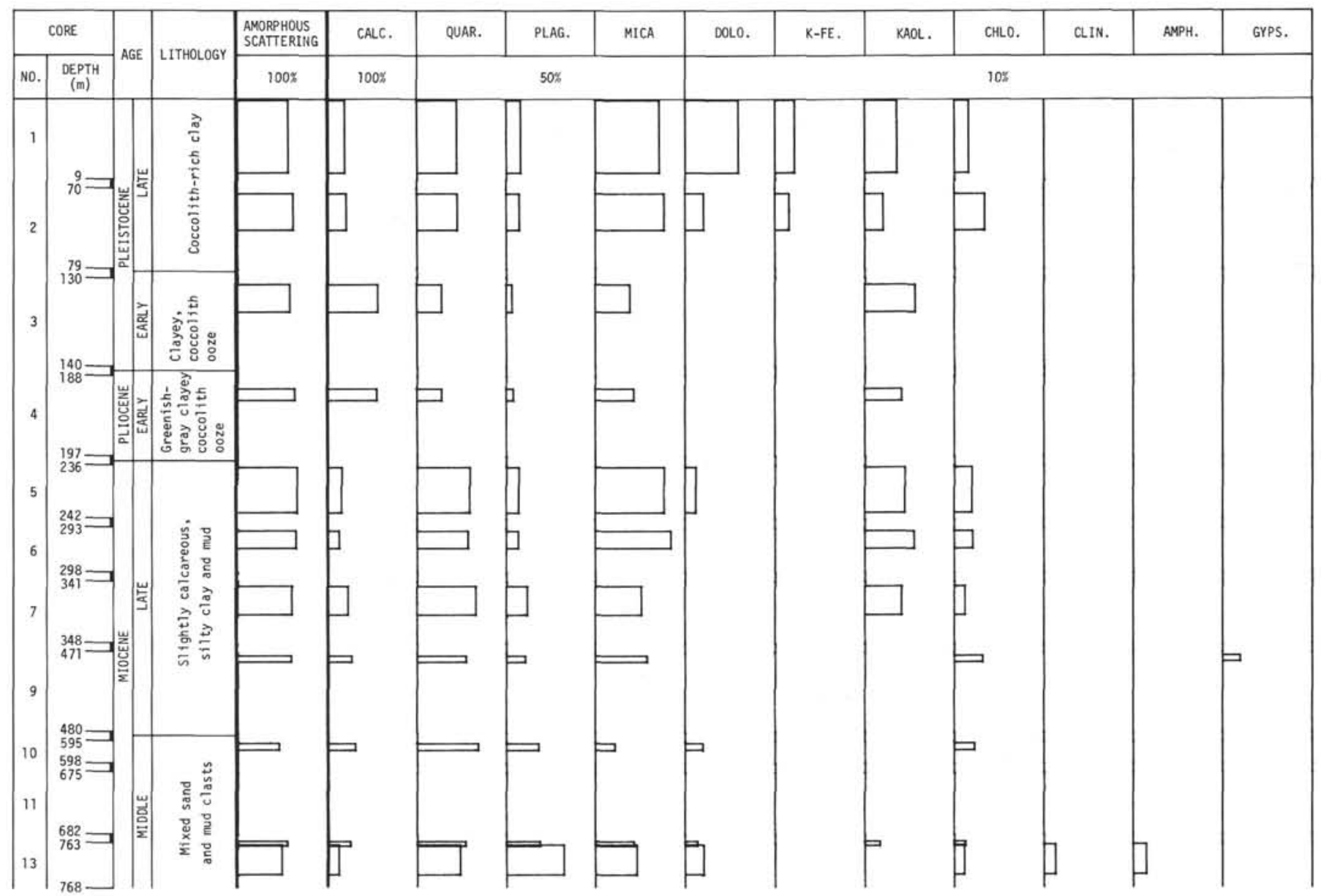

Figure 11. Site 90 bulk samples. 


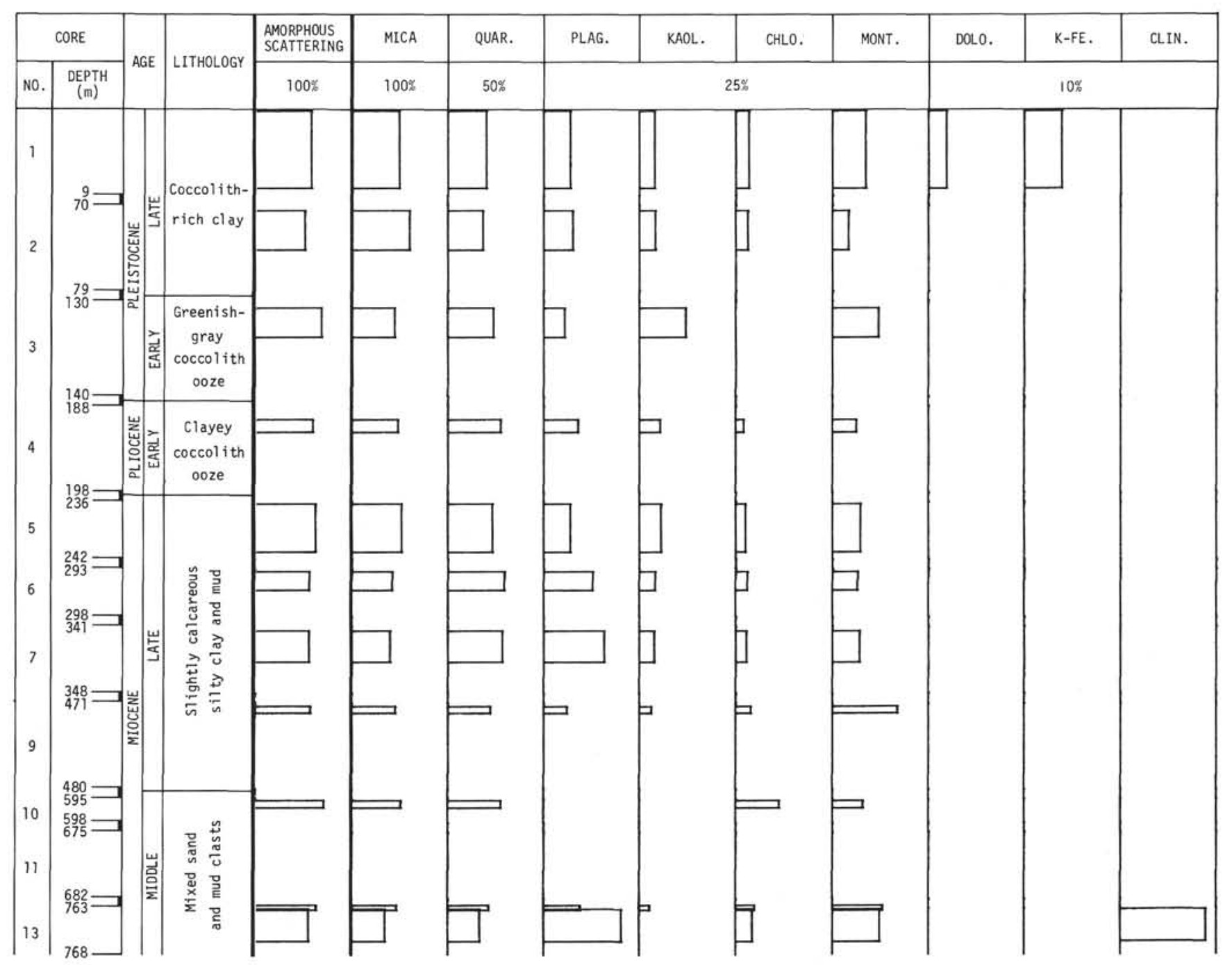

Figure 12. Site $90<2 \mu \mathrm{m}$ fractions. 
X-RAY MINERALOGY STUDIES, GULF OF MEXICO

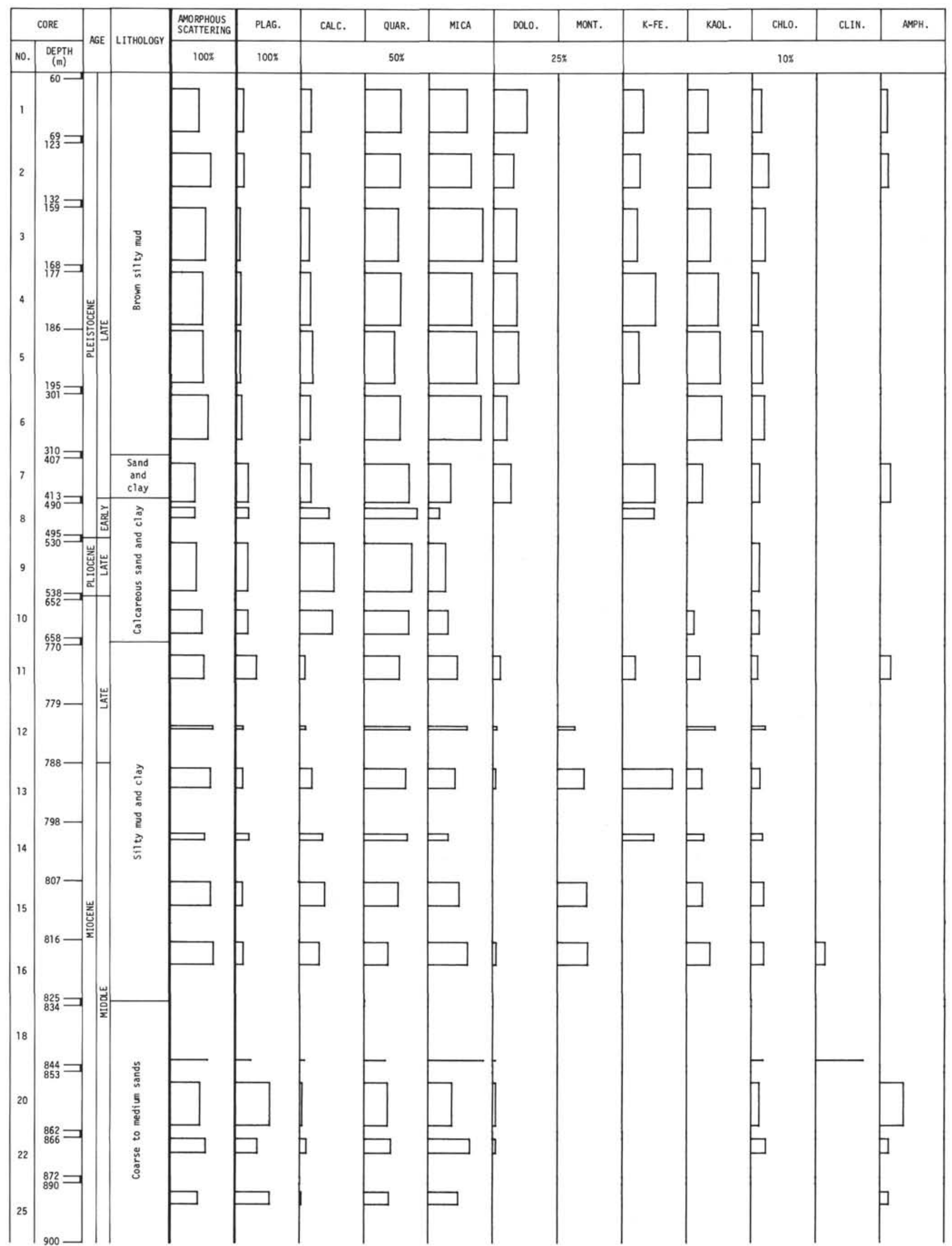

Figure 13. Site 91 bulk samples. 


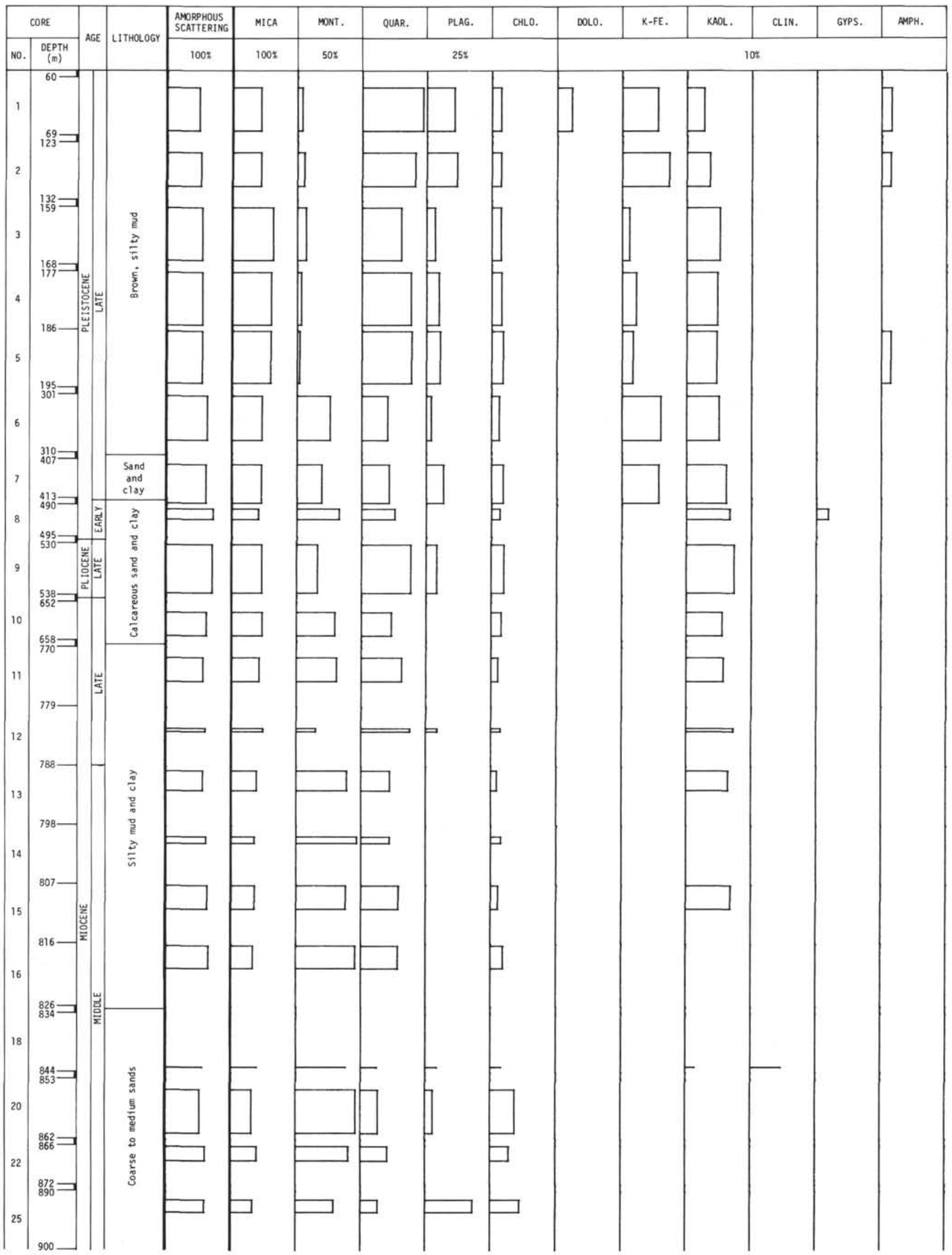

Figure 14. Site $91<2 \mu \mathrm{m}$ fractions. 


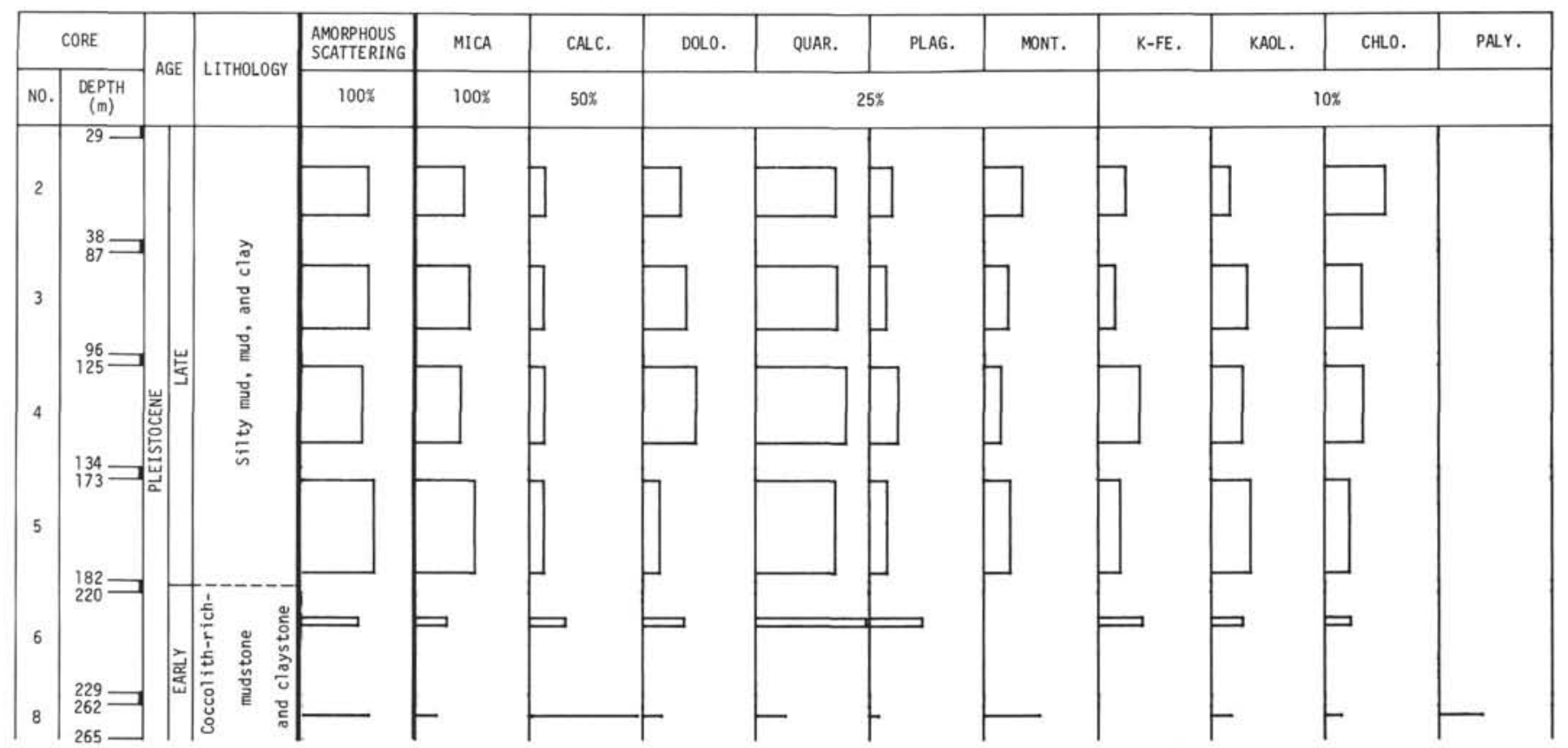

Figure 15. Site 92 bulk samples.

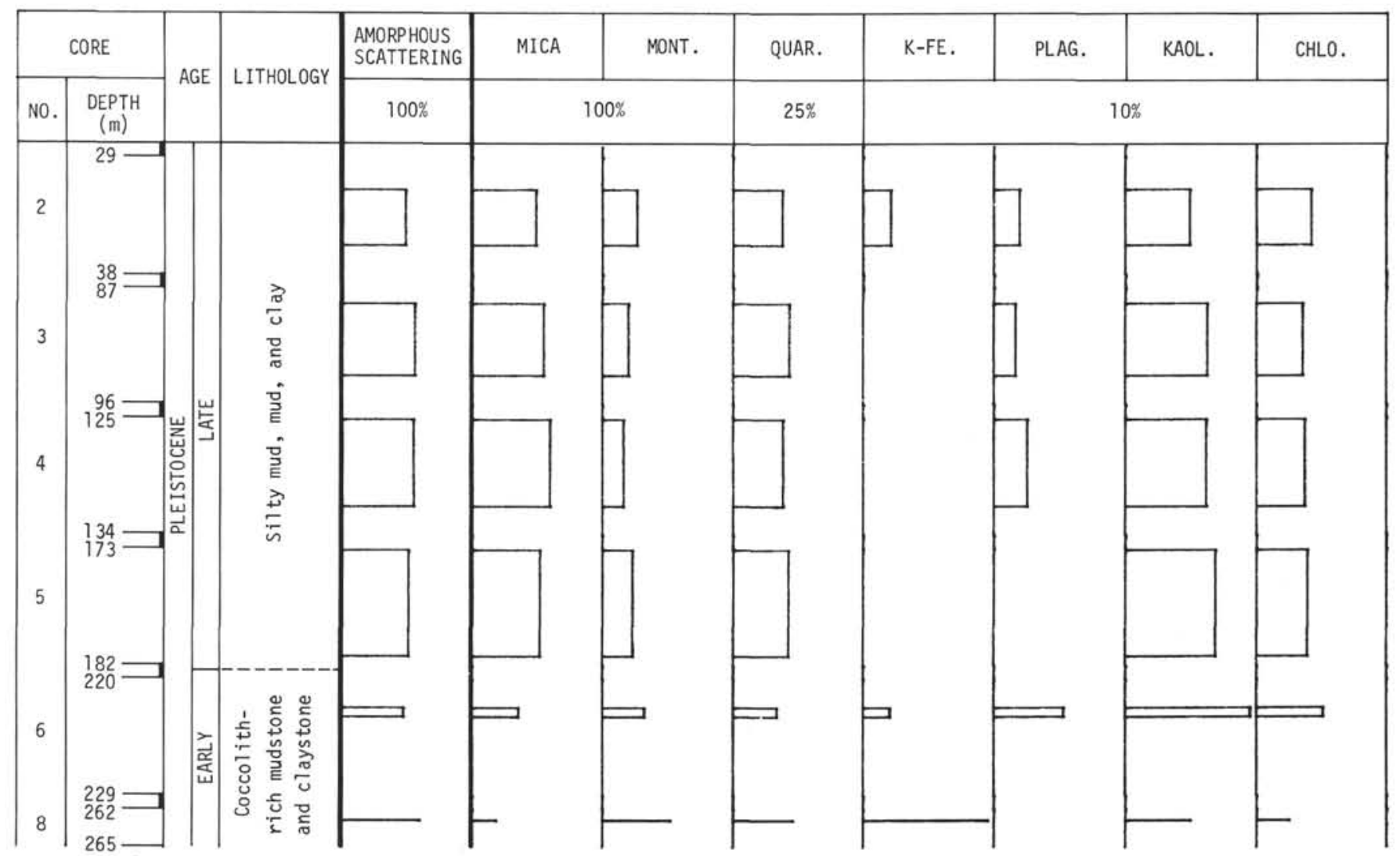

Figure 16. Site $92<2 \mu \mathrm{m}$ fractions.

\begin{tabular}{|c|c|c|c|c|c|c|c|c|c|c|c|c|c|}
\hline \multicolumn{2}{|c|}{ CORE } & \multirow{2}{*}{ AGE } & \multirow{2}{*}{ LITHOLOGY } & $\begin{array}{l}\text { AMORPHOUS } \\
\text { SCATTERING }\end{array}$ & CALC. & ARAG. & MICA & DOLO. & QUAR. & PLAG. & KAOL. & MONT. & CHLO. \\
\hline No. & $\begin{array}{c}\text { DEPTH } \\
(\mathrm{m})\end{array}$ & & & $100 \%$ & $100 \%$ & $50 \%$ & $25 \%$ & \multicolumn{6}{|c|}{$10 \%$} \\
\hline 1 & 2 & * & $\begin{array}{l}\text { Clayey } \\
\text { coccolith } \\
\text { ooze }\end{array}$ & & & & & & & & & & \\
\hline
\end{tabular}

Figure 17. Site 93 bulk samples. 


\begin{tabular}{|c|c|c|c|c|c|c|c|c|c|c|c|c|}
\hline \multicolumn{2}{|c|}{ CORE } & \multirow{2}{*}{ AGE } & \multirow{2}{*}{ LITHOLOGY } & $\begin{array}{l}\text { AMORPHOUS } \\
\text { SCATTERING }\end{array}$ & MONT. & MICA & PALY. & QUAR. & PLAG. & KAOL. & CHLO. & GYPS. \\
\hline No. & $\begin{array}{l}\text { DEPTH } \\
\text { (m) }\end{array}$ & & & $100 \%$ & $100 \%$ & \multicolumn{2}{|c|}{$25 \%$} & \multicolumn{5}{|c|}{$10 \%$} \\
\hline 1 & $2-$ & * & $\begin{array}{l}\text { Clayey } \\
\text { coccolith } \\
\text { ooze }\end{array}$ & & & & & & & & & \\
\hline
\end{tabular}

* holocene and LATE miocene

Figure 18. Site $93<2$ um fractions.

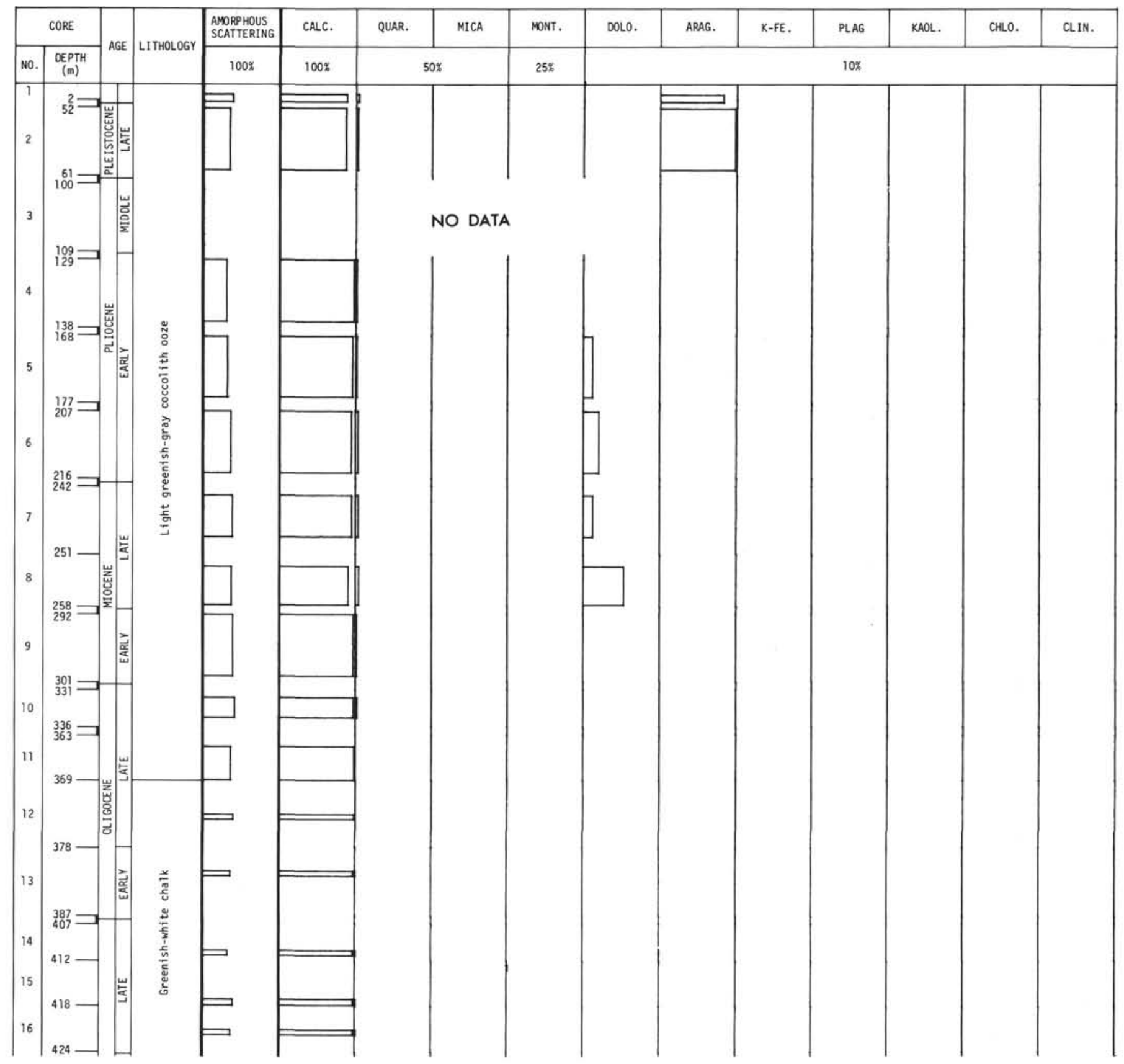

Figure 19. Site 94 bulk samples. 


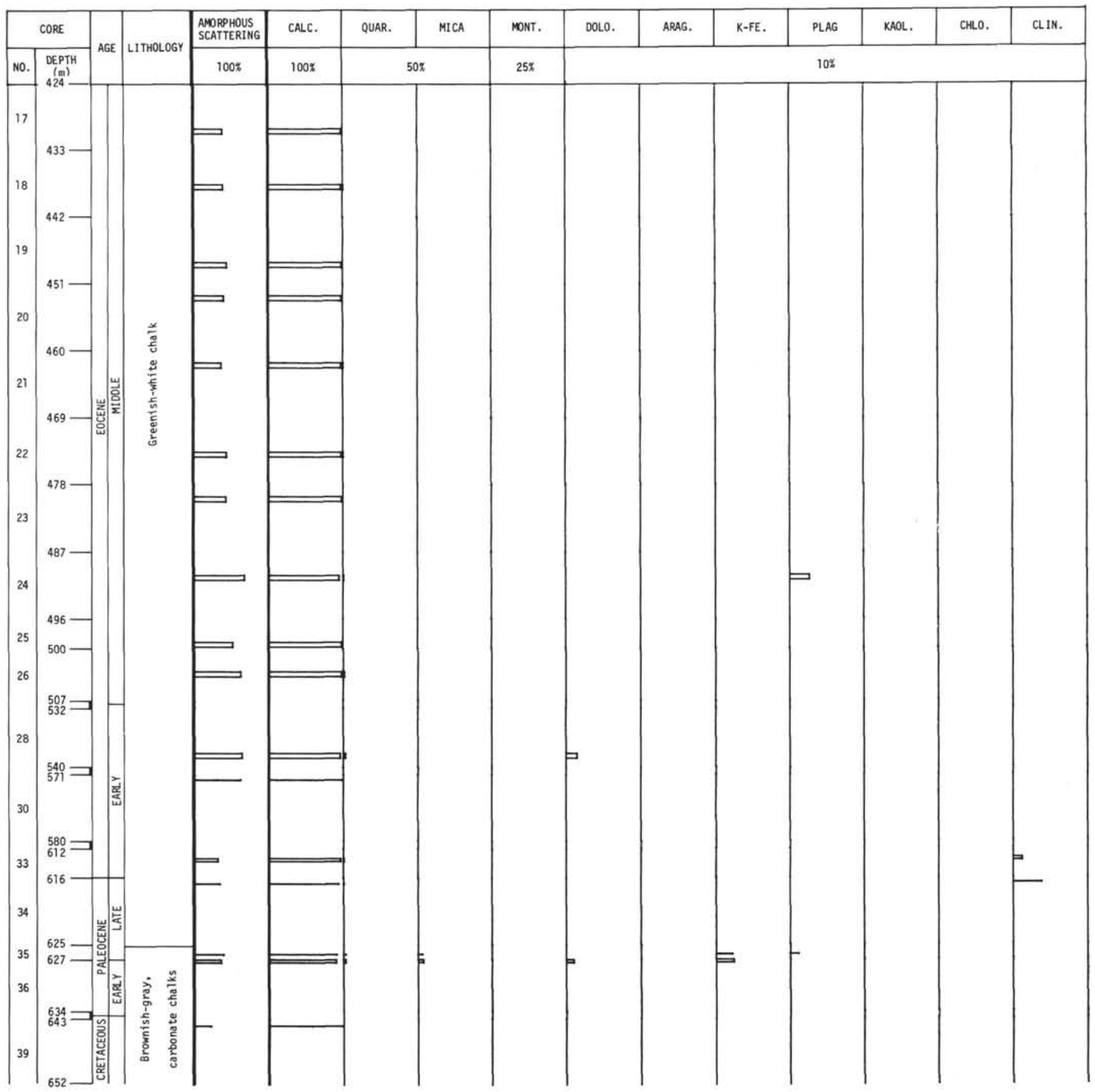

Figure 19. (Continued). 


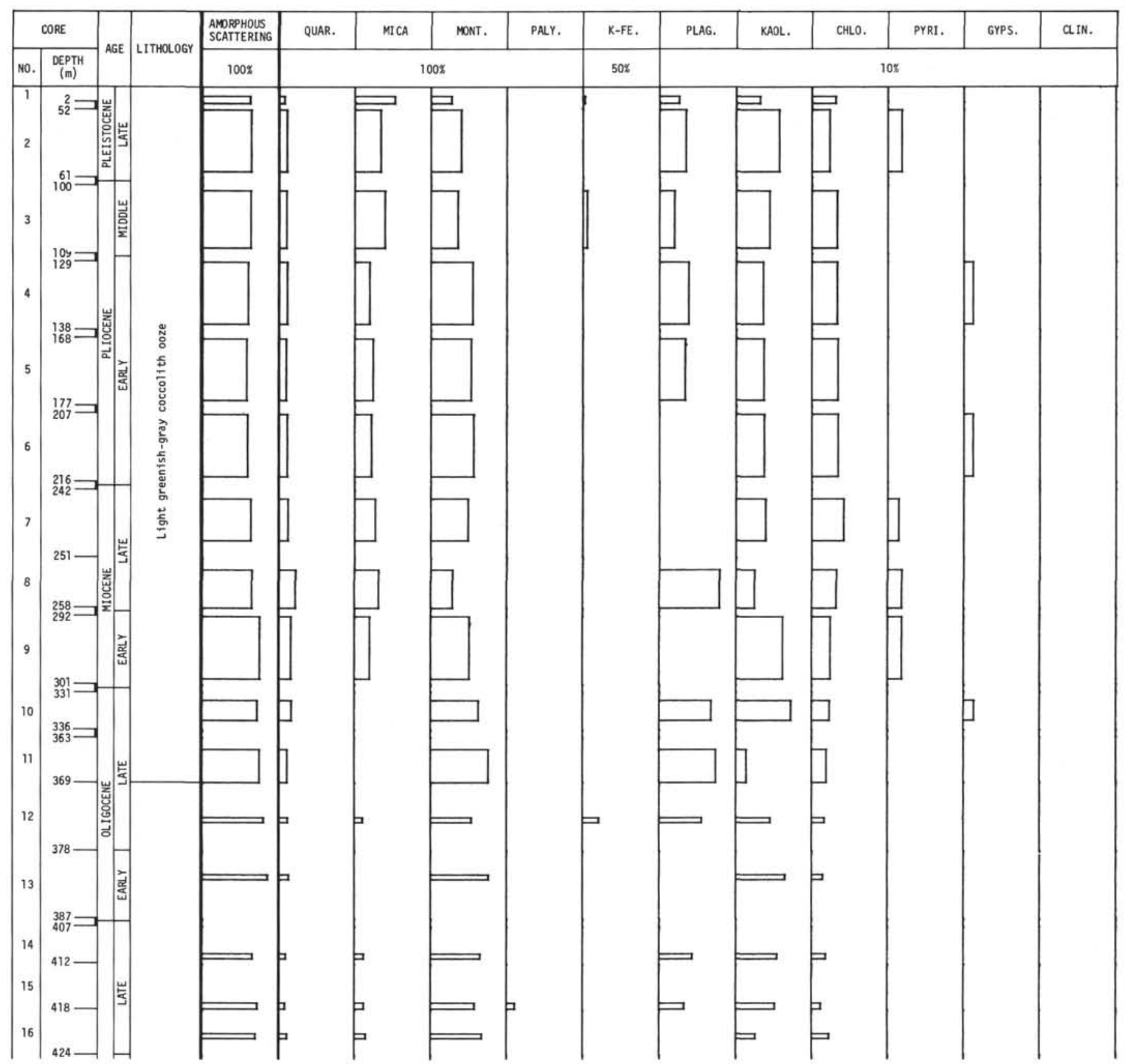

Figure 20. Site $94<2 \mu$ m fractions. 


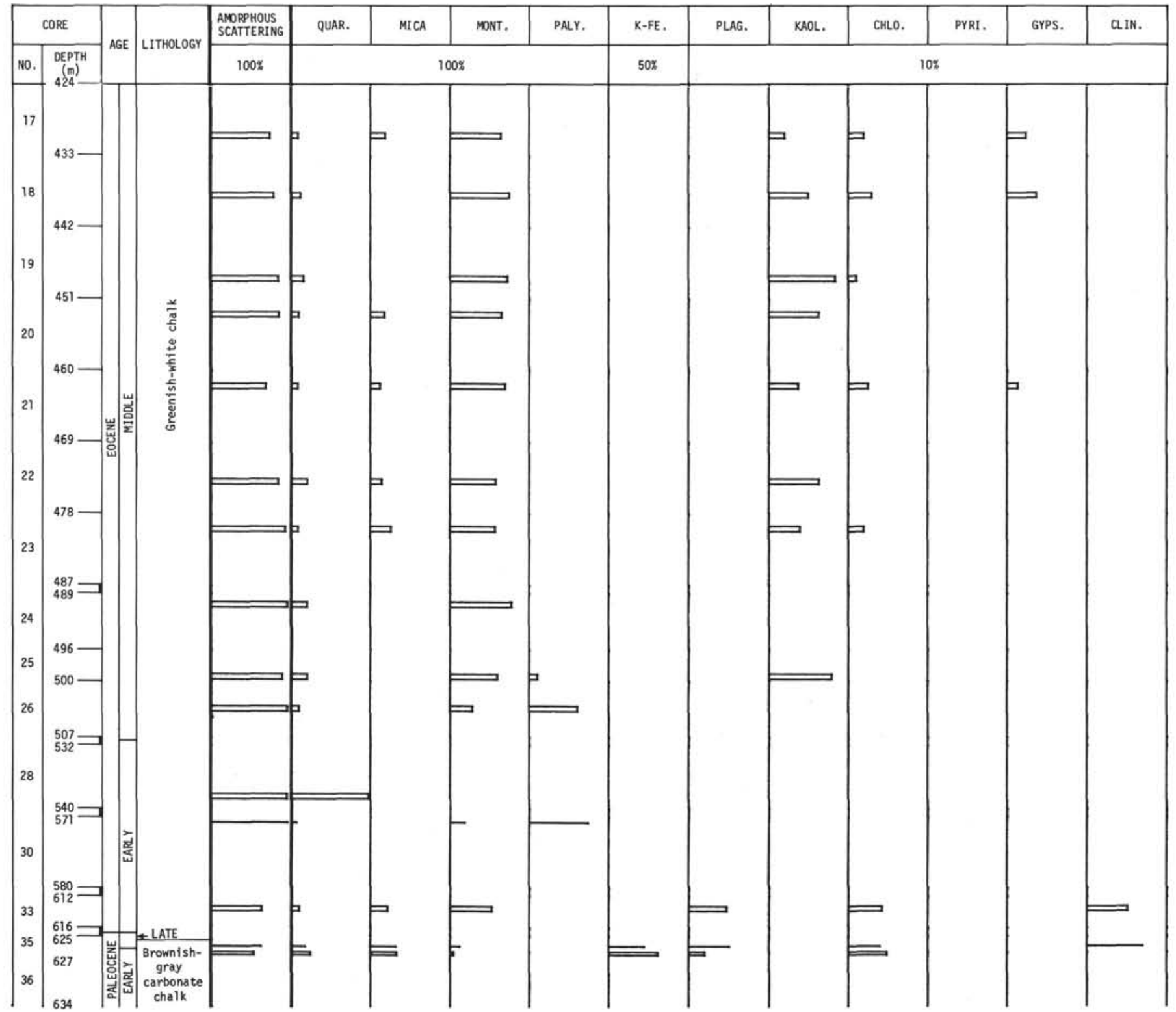

Figure 20. (Continued) 


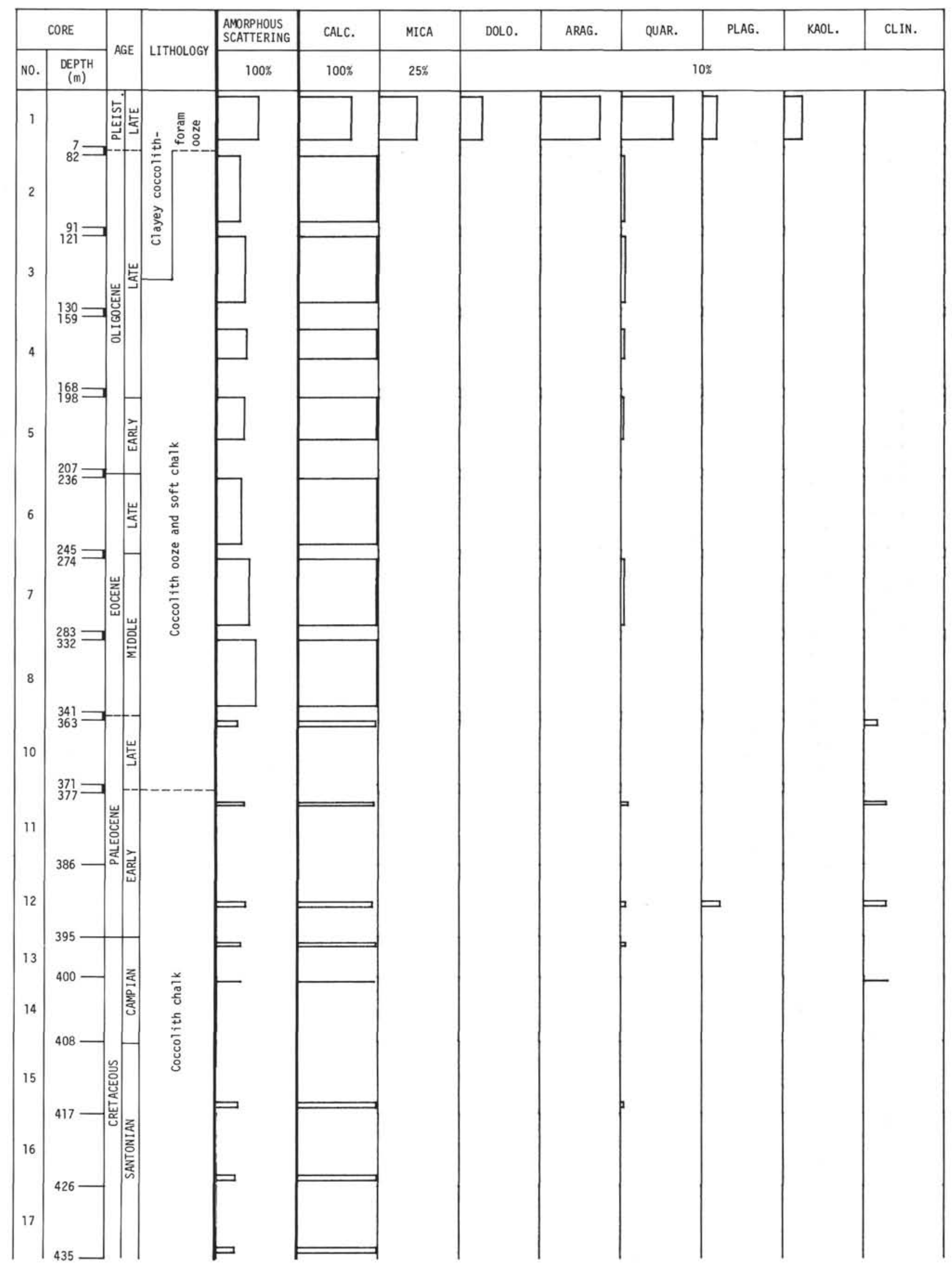

Figure 21. Site 95 bulk samples. 


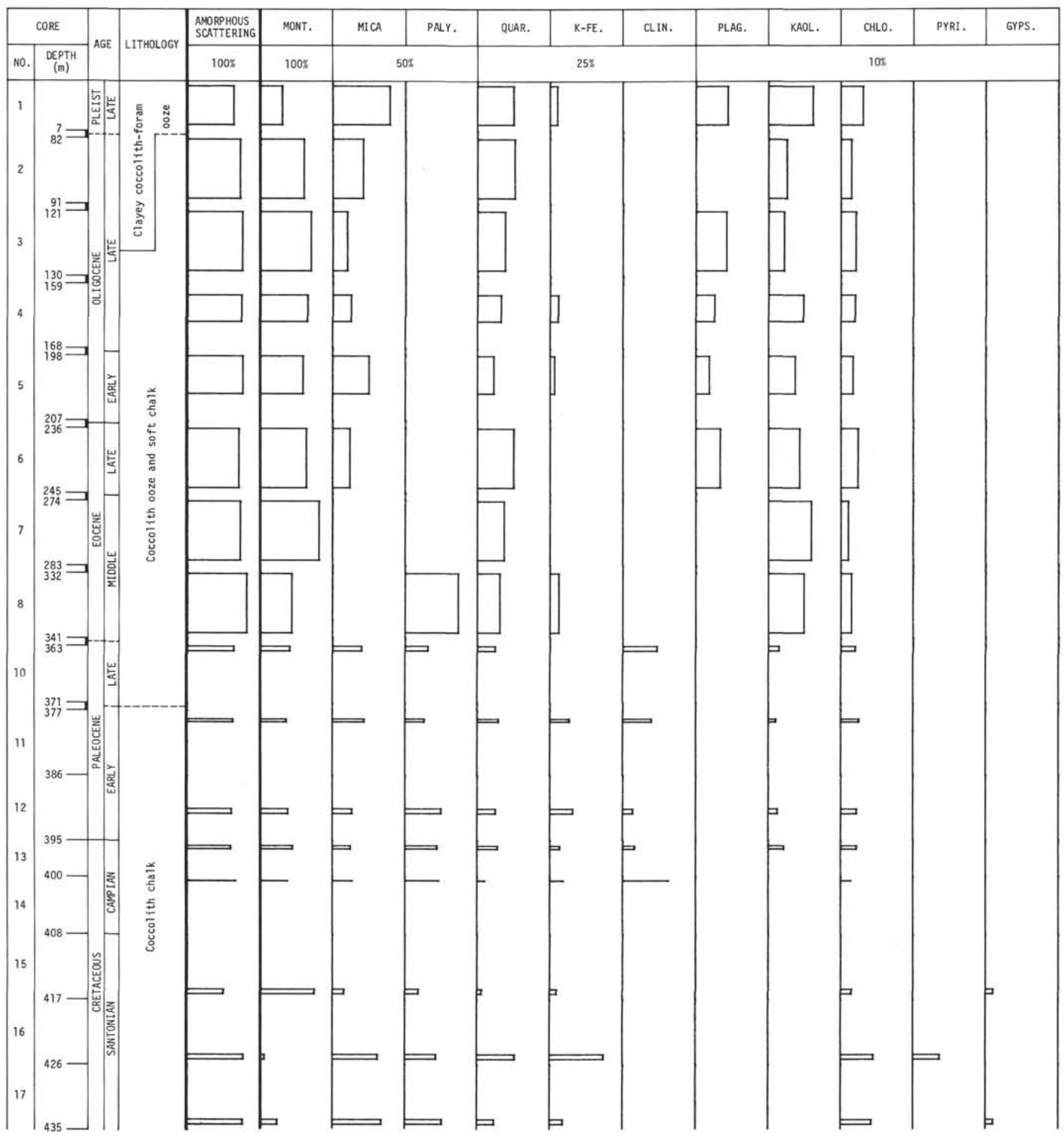

Figure 22. Site $95<2 \mu$ m fractions. 


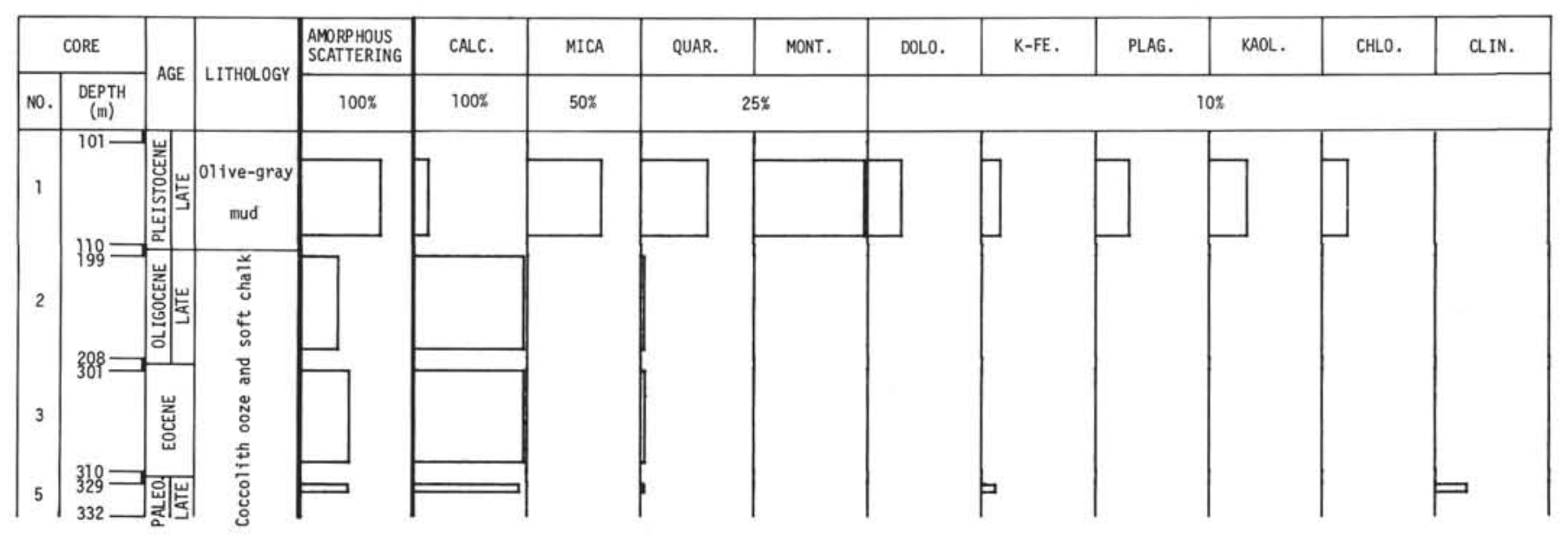

Figure 23. Site 96 bulk samples.

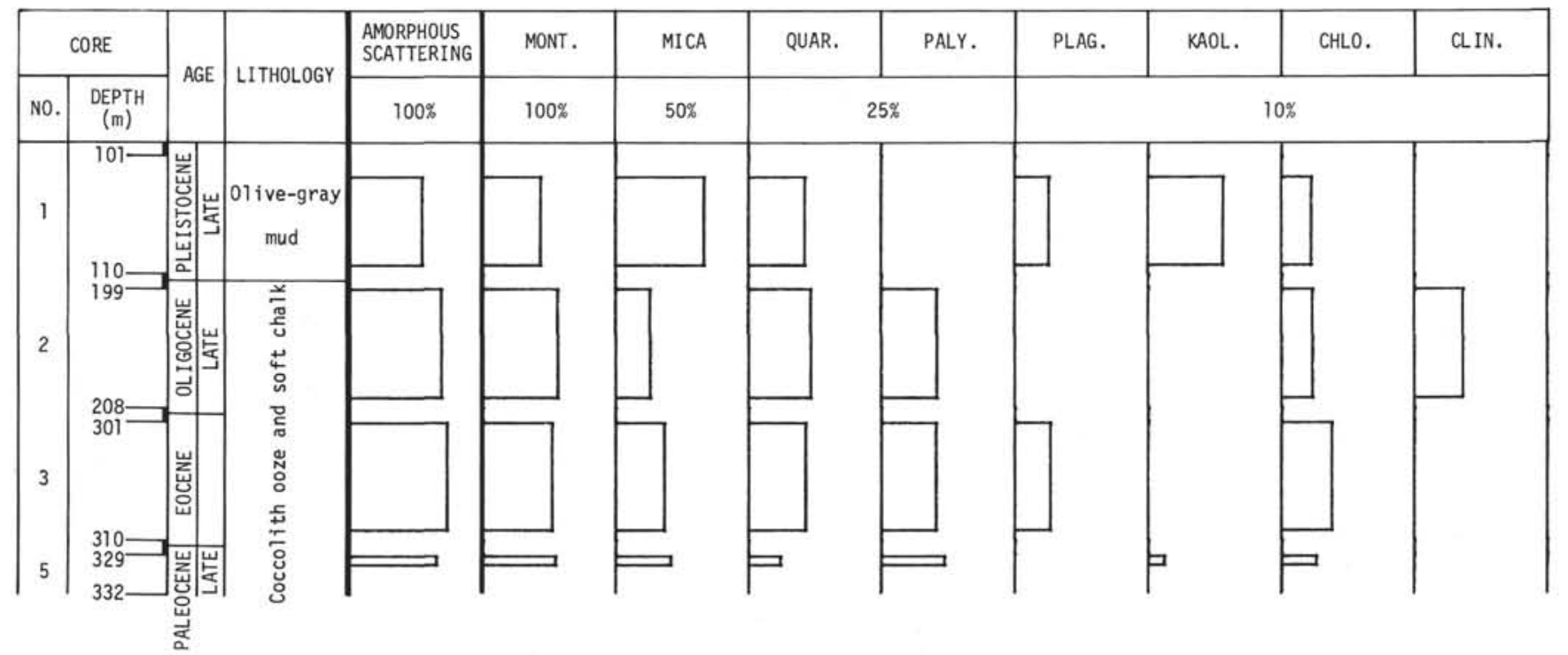

Figure 24. Site $96<2 \mu \mathrm{m}$ fractions. 


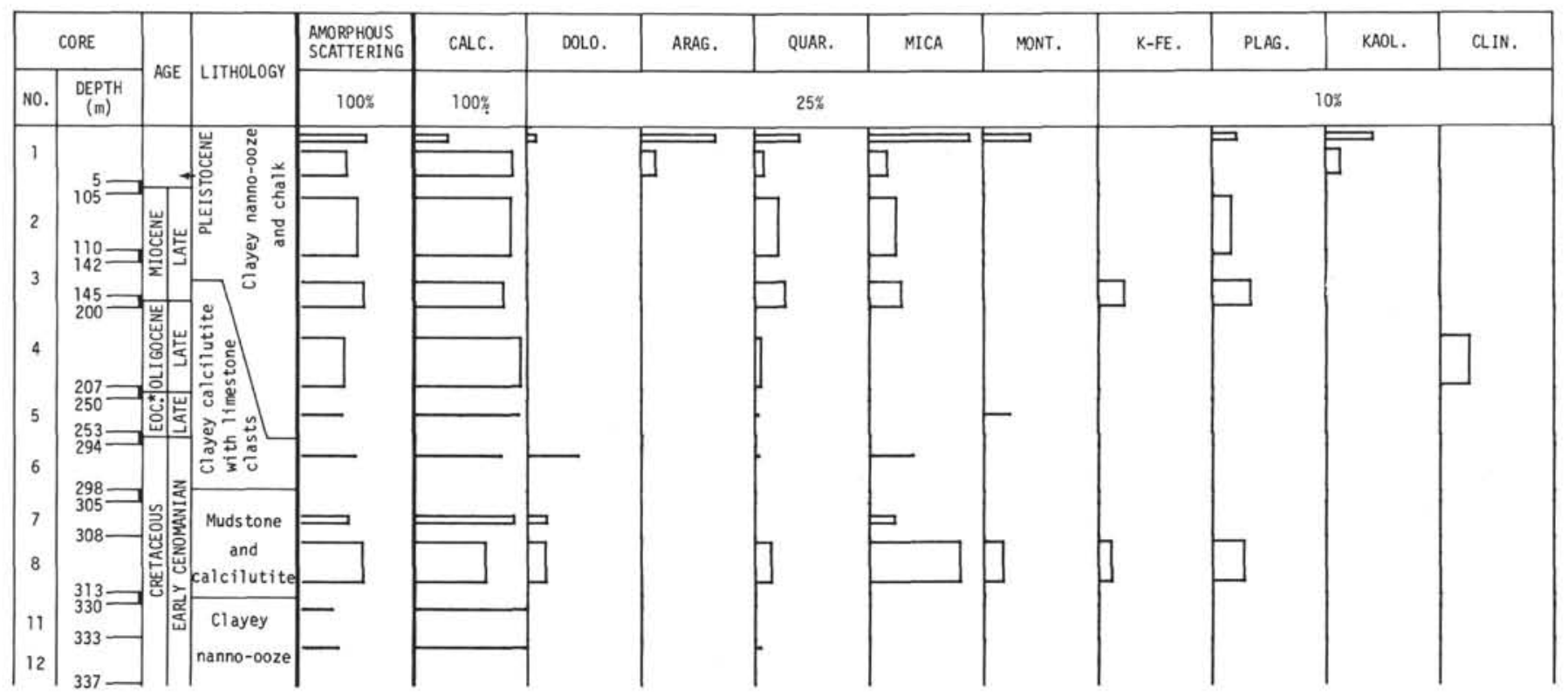

Figure 25. Site 97 bulk samples.

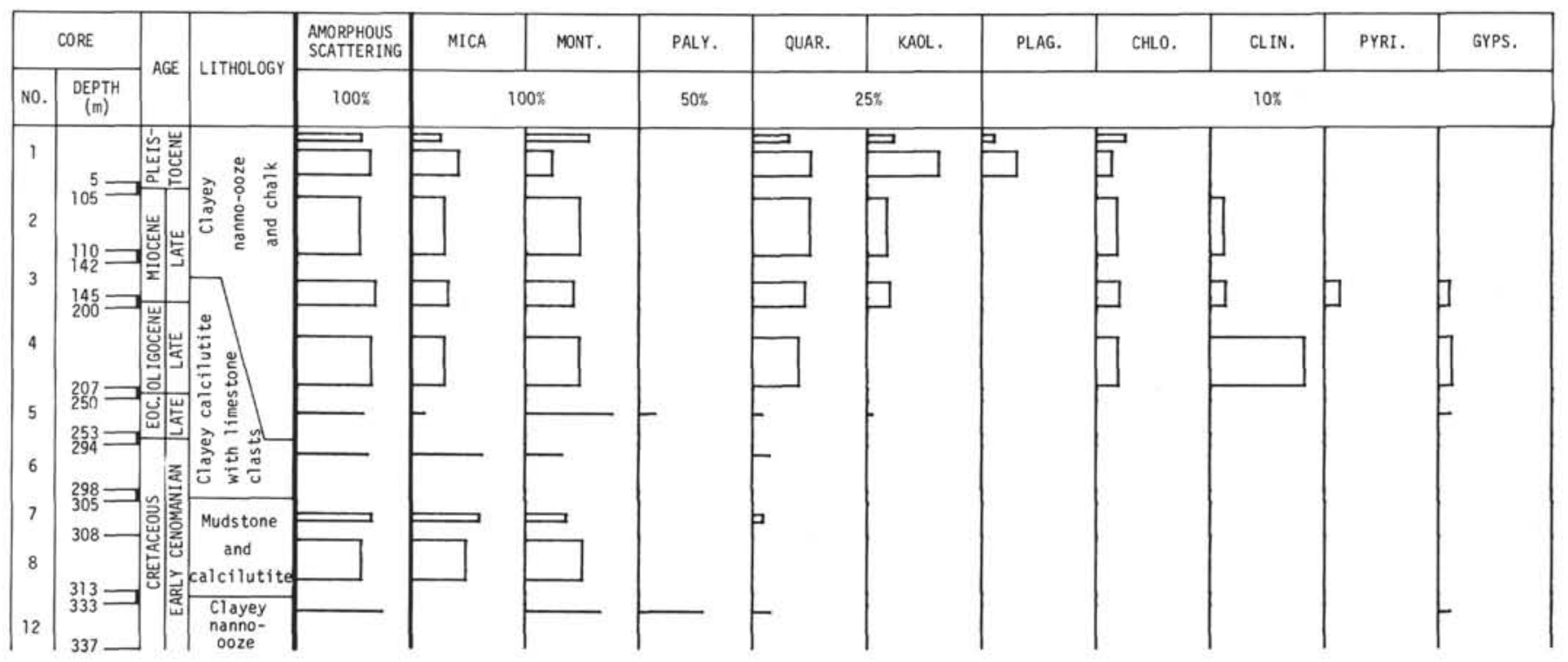

Figure 26. Site $97<2 \mu \mathrm{m}$ fractions. 